\title{
Determinants of Control-Key Responding in an Operant Resurgence Procedure
}

\author{
Anthony C. Oliver \\ acoliver@mix.wvu.edu
}

Follow this and additional works at: https://researchrepository.wvu.edu/etd

Part of the Experimental Analysis of Behavior Commons

\section{Recommended Citation}

Oliver, Anthony C., "Determinants of Control-Key Responding in an Operant Resurgence Procedure" (2019). Graduate Theses, Dissertations, and Problem Reports. 3842.

https://researchrepository.wvu.edu/etd/3842

This Dissertation is protected by copyright and/or related rights. It has been brought to you by the The Research Repository @ WVU with permission from the rights-holder(s). You are free to use this Dissertation in any way that is permitted by the copyright and related rights legislation that applies to your use. For other uses you must obtain permission from the rights-holder(s) directly, unless additional rights are indicated by a Creative Commons license in the record and/ or on the work itself. This Dissertation has been accepted for inclusion in WVU Graduate Theses, Dissertations, and Problem Reports collection by an authorized administrator of The Research Repository @ WVU.

For more information, please contact researchrepository@mail.wvu.edu. 
2019

Determinants of Control-Key Responding in an Operant Resurgence Procedure

Anthony C. Oliver

Follow this and additional works at: https://researchrepository.wvu.edu/etd

Part of the Experimental Analysis of Behavior Commons 
Determinants of Control-Key Responding in an Operant Resurgence Procedure Anthony C. Oliver

Dissertation Submitted to the Eberly College of Arts and Sciences at West Virginia University in partial fulfillment of the requirements for the degree of

\author{
Doctor of Philosophy \\ in \\ Psychology
}

\author{
Kennon A. Lattal, Ph.D., Chair \\ Melissa D. Blank, Ph.D. \\ Adam H. Doughty, PhD. \\ Kathryn M. Kestner, Ph.D. \\ Michael Perone, Ph.D. \\ Department of Psychology
}

Morgantown, West Virginia

2019

Keywords: resurgence; reinforcement history; hierarchical resurgence; pigeons; rats Copyright 2019 Anthony Oliver 


\begin{abstract}
Determinants of Control-Key Responding in an Operant Resurgence Procedure
\end{abstract}

Anthony C. Oliver

The Control response in a resurgence procedure has been described as the "gold standard” in isolating resurgence from other extinction-induced phenomena. Although Control responding is not generally observed during the resurgence test phase of animal-resurgence procedures, it is common to see such responding in human-operant resurgence procedures. Thus, it is necessary to identify the conditions under which Control responding may be observed. Using pigeons and rats as subjects, the current series of experiments examined the determinants of Control-operandum responding in a resurgence procedure. Experiment 1 assessed whether behavior with a pre-experimental reinforcement history would resurge if the Target response was maintained by a differential reinforcement of other (DRO) contingency. Experiment 2a was a systematic replication of Experiment 1; however, a four-phase resurgence procedure was used. This fourth phase was used to establish a reinforcement history for a simulated Control response with a reinforcement history. Experiment 2b, was a systematic replication of Experiment 2a, except this experiment was conducted with rats and subjects and using a within-session resurgence procedure. Finally, Experiment 3 was a within-subject replication of Experiment 2a and $2 \mathrm{~b}$ that was designed to evaluate how the conditions under which a modeled Control response was maintained on the subsequence resurgence of that response using a four-phase resurgence procedure. The results of these four experiments indicate the following: 1) If Control responding is observed during a resurgence procedure, then the likely source of such responding is a previous history of reinforcement, 2) Resurgence of a response is dependent on the conditions under which that response was maintained prior to its elimination, and 3)The resurgence of multiple responses is hierarchical, with the order and length of training a determining factor in the magnitude and probability of resurgence. The results of all four experiments are discussed in relation to the interpretation of Control responding during the RT phase, hierarchical resurgence, and potential areas of application. 


\section{Acknowledgements}

Thank you to Andy Lattal for giving me the many opportunities to challenge myself during the last several years. Andy’s mentorship and guidance were instrumental in helping me become the person I am. I will be forever grateful for the opportunity to work with Andy during my graduate career.

I would like to thank the members of my committee for their feedback and guidance in the competition of this dissertation. Thank you, Andy Lattal, Mike Perone, Melissa Blank, Katie Kestner, and Adam Doughty, your input and comments were invaluable in this process. In addition to my committee members, I am indebted to the graduate and undergraduate students in the Lattal lab who helped run each of these experiments.

Finally, I could not have done any of this without the support of my family and friends. Leigh has been the most incredibly supportive and amazing partner in this journey. Thank you to my parents and siblings who continually supported and encouraged me in my pursuit of a doctoral degree. I will be forever grateful for your support and encouragement. 


\section{Table of Contents}

ABSTRACT

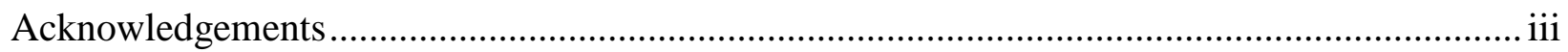

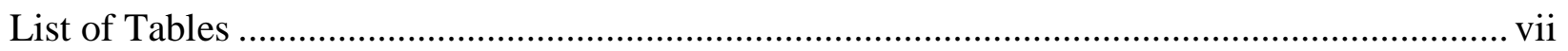

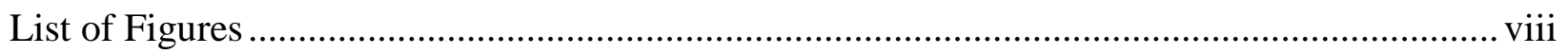

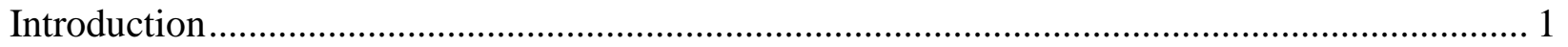

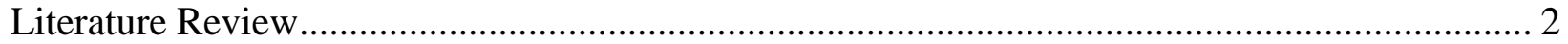

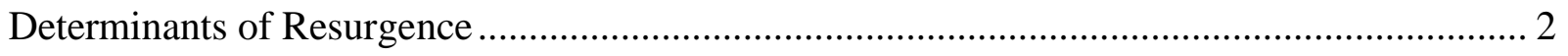

The Control Response and its Implications ................................................................. 5

Resurgence of Multiple Responses: Implications for Understanding Control Responding ....... 9

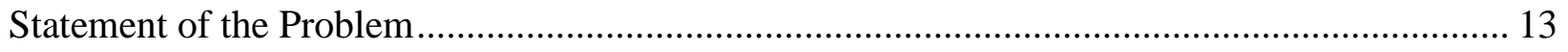

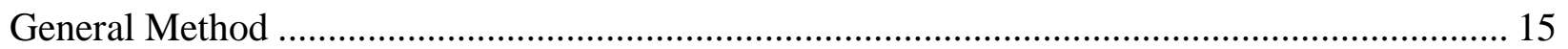

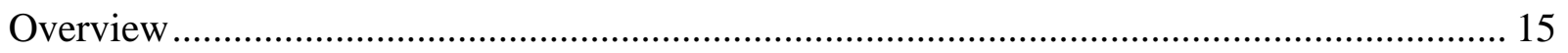

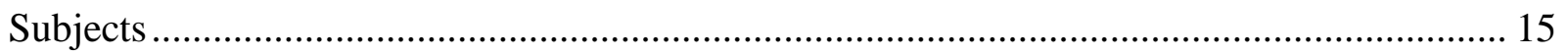

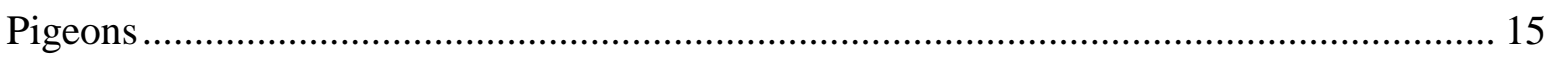

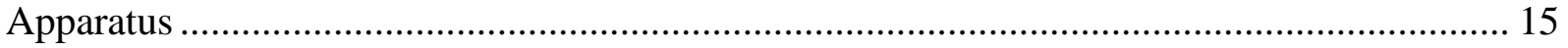

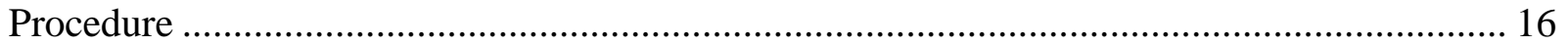

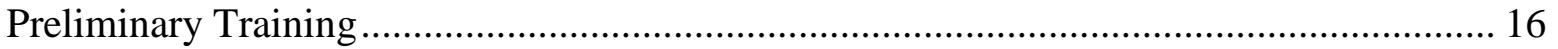

Alternative Reinforcement (AR) Phase .................................................................... 16

Resurgence Test (RT) Phase .................................................................................. 17

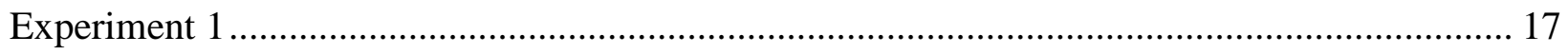

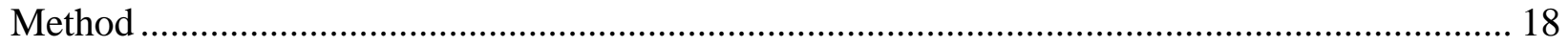

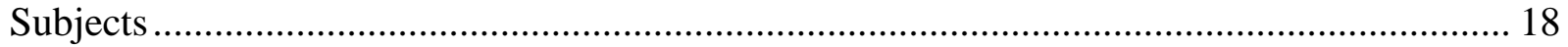

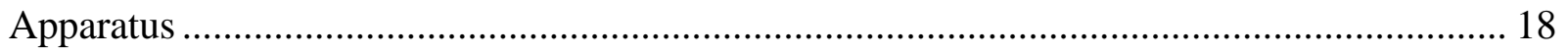

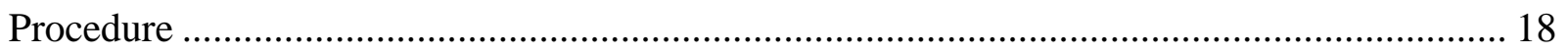

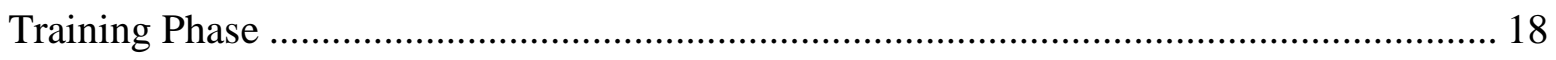

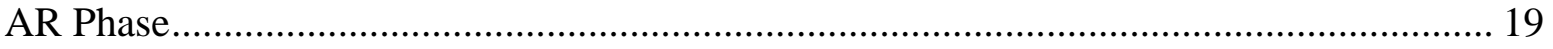

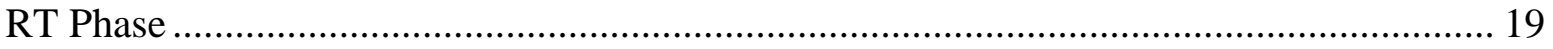

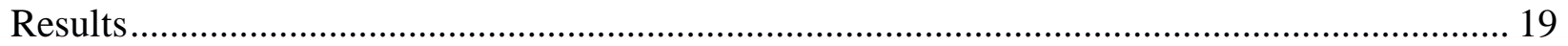

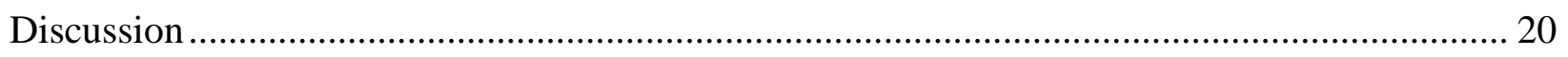

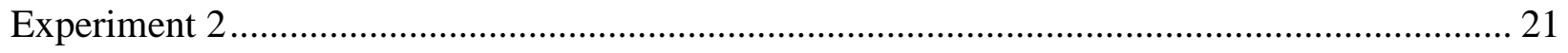

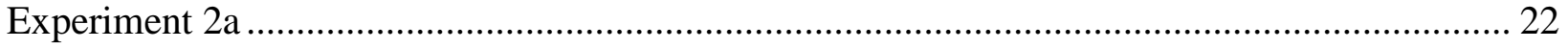

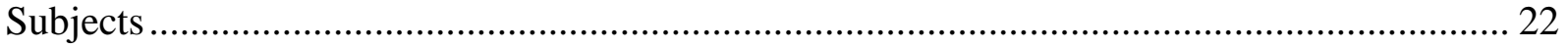




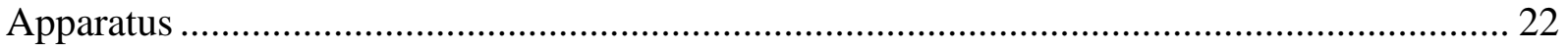

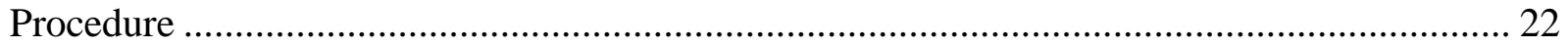

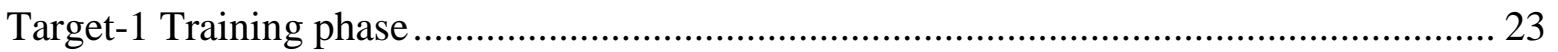

Target-2 Training phase ................................................................................................ 23

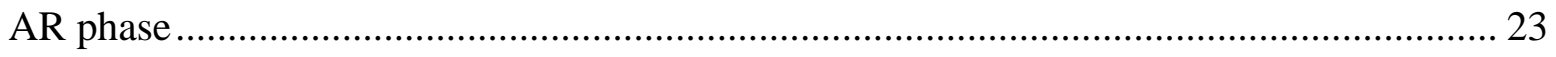

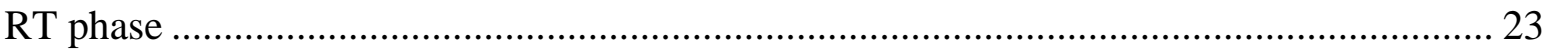

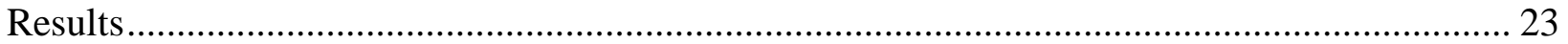

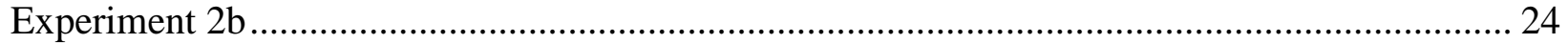

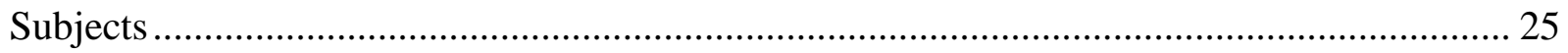

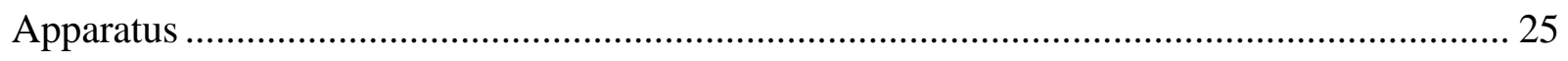

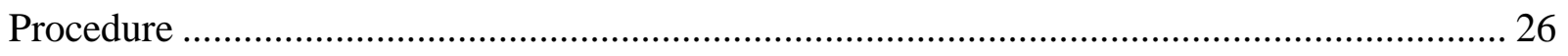

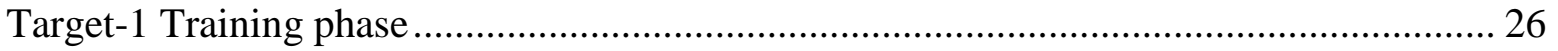

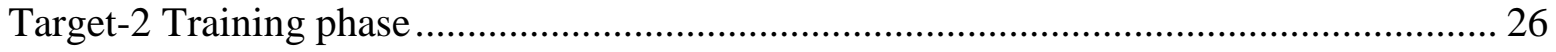

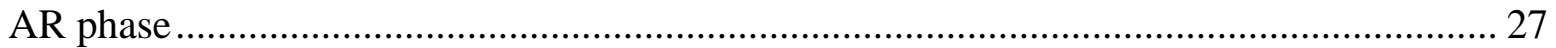

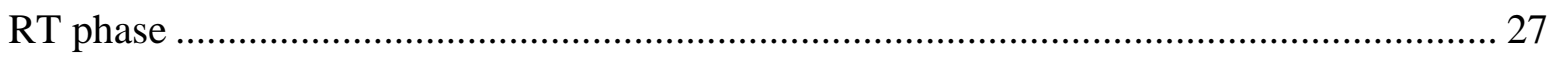

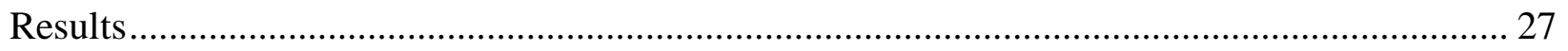

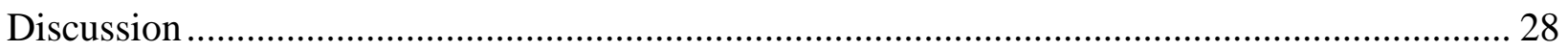

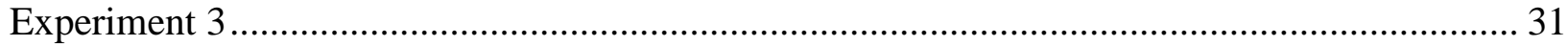

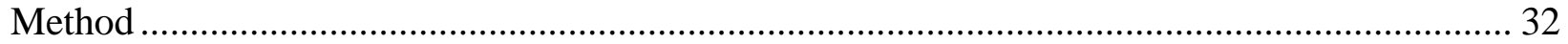

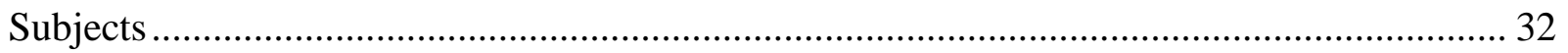

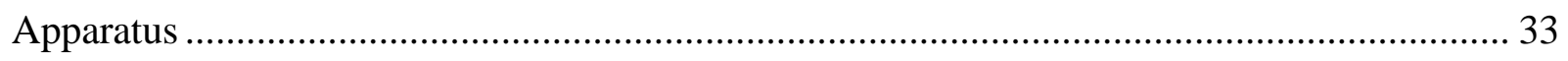

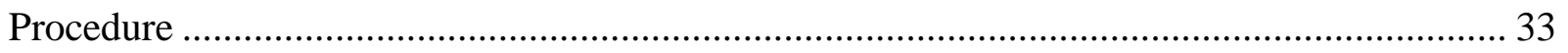

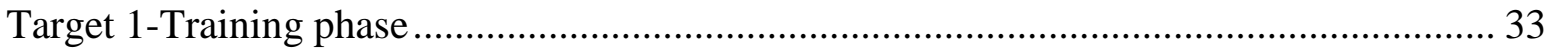

Target-2 Training phase ............................................................................................ 34

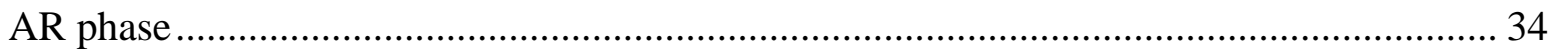

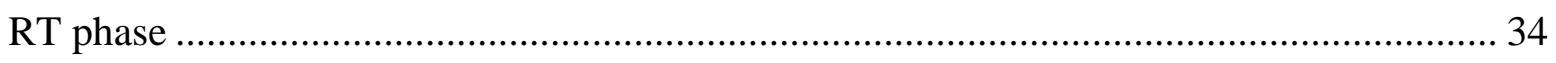

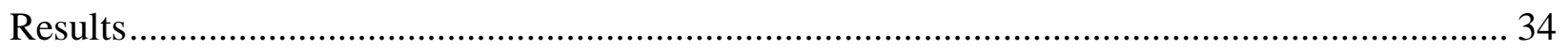

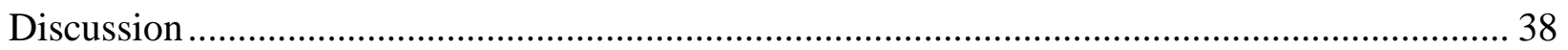

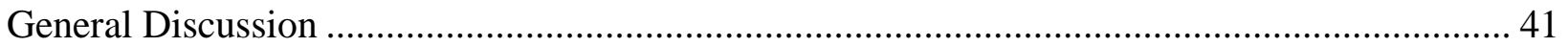

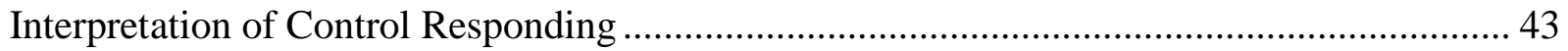

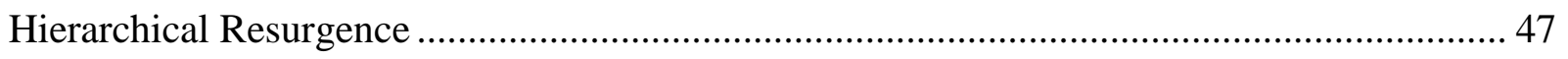

Levels of Control Responding: Differences in Human and Nonhuman Resurgence

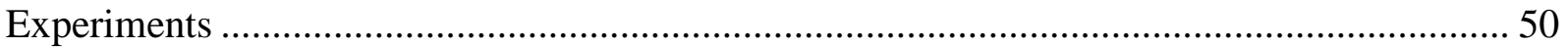




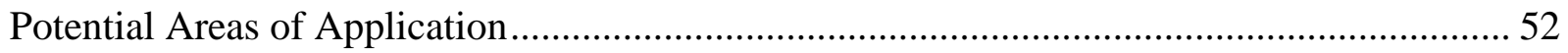

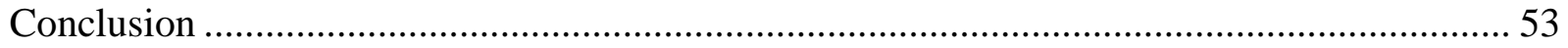

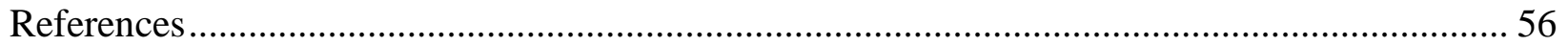

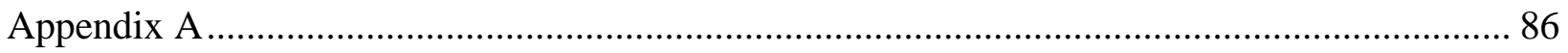

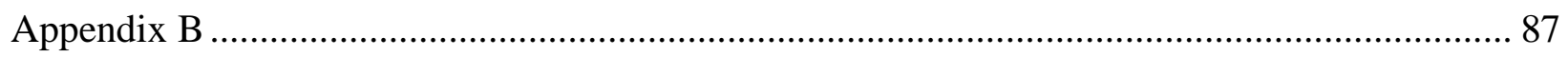




\section{List of Tables}

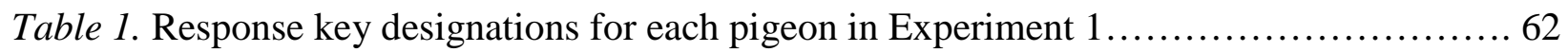

Table 2. Response key designations for each pigeon in Experiment 2....................... 63

Table 3. Response key designations for each pigeon for each component in Experiment 3..... 64

Table 4. Mean reinforcement rates for each component for each experiment in Experiment 3...65 


\section{List of Figures}

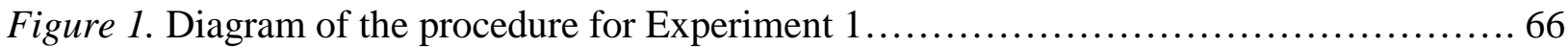

Figure 2. Response rates on the Alternative, Target, and Control keys during each phase for each

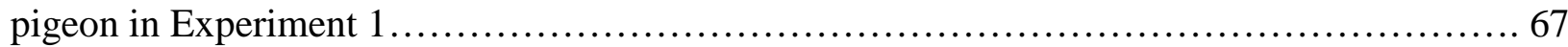

Figure 3. Control and Target Response rates for the last three sessions of the Alternativereinforcement phase and all sessions of the RT phase for all pigeons in Experiment 1 ........ 68

Figure 4. Diagram of the procedure for Experiment 2a................................69

Figure 5. Responses per minute of the Alternative, Target 1, Target 2, and Control keys for all pigeons in Experiment 2a.......................................................70

Figure 6. Summary figures for the last three sessions for the Alternative Reinforcement phase and all sessions of the Resurgence Test phase for all pigeons in Experiment 2a............... 71

Figure 7. Diagram of the procedure used in Experiment 2b.............................. 72

Figure 8. Number of Target 1, Target 2, and Control responses during the RT phase for each rat in Experiment $2 \mathrm{~b}$.

Figure 9. Frequency of Target 1, Target 2, and Control responses during successive 1-min bins of the RT phase of each experimental session of Experiment 2 b.......................... 74

Figure 10. Diagram of the procedure used in Experiment 3............................ 75

Figure 11. Rates of the VRT1, VRT2, and ALT VR responses during each phase of Experiment

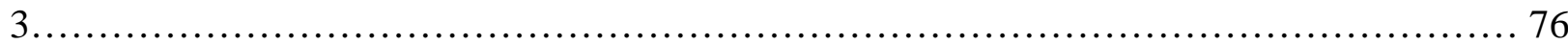

Figure 12. Response rates of VRT1 and VRT2 during the last three sessions of the AR phase and

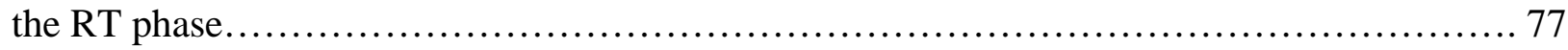

Figure 13. Total responses (key pecks) on the VRT1 and VRT2 keys during the last three sessions of the Alternative-Reinforcement phase and first three days of the Resurgence-Test

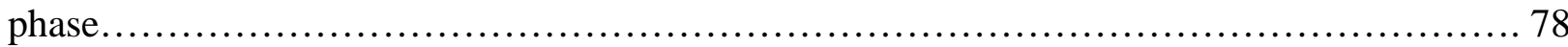

Figure 14. Cumulative record of VRT1 and VRT2 responses during the first session of the first RT phase and the first session of the replication........................................ 79

Figure 15. Response rates of the DROT1, DROT2, and ALDRO for each phase for each pigeon

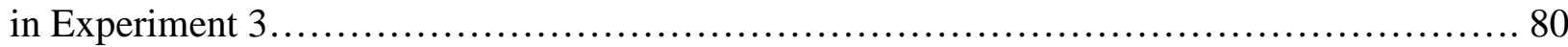

Figure 16. Response rates of DROT1 and DROT2 during the last three sessions of the AR phase

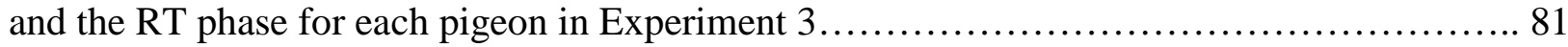

Figure 17. Total responses (key pecks) on the DROT1 and DROT2 keys during the last three sessions of the Alternative-Reinforcement phase and first three days of the Resurgence-Test

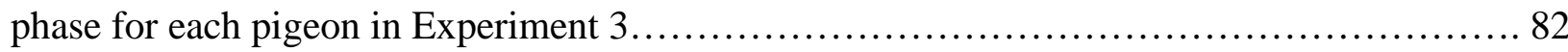


Figure 18. Cumulative record of DROT1 and DROT2 responses during the first session of the first RT phase (left panel) and the first session of the replication (right panel) for each pigeon in

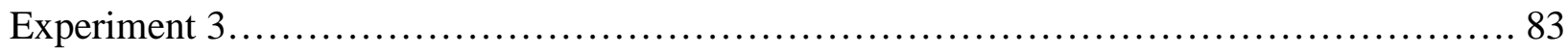

Figure 19. Response rates of DROT1 and VRT1 during the last three sessions of the AR phase

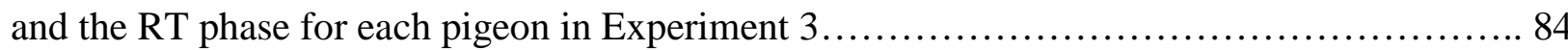

Figure 20. Total responses (key pecks) on the DROT1 and VRT1 keys during the last three sessions of the Alternative-Reinforcement phase and first three days of the Resurgence-Test phase for each pigeon in Experiment 3.............................................. 85

Figure B1. Cumulative record of Alternative, Target-1, Target-2, and Control responses during

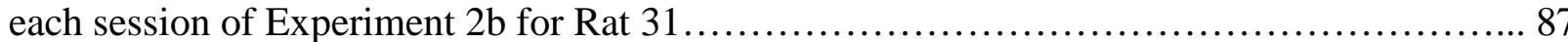

Figure B2. Cumulative record of Alternative, Target-1, Target-2, and Control responses during

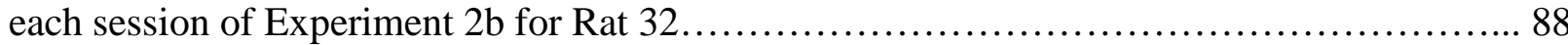

Figure B3. Cumulative record of Alternative, Target-1, Target-2, and Control responses during

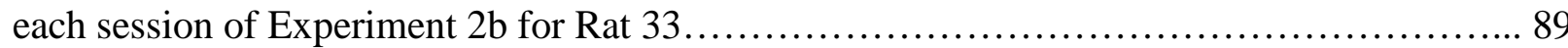

Figure B4. Cumulative record of Alternative, Target-1, Target-2, and Control responses during

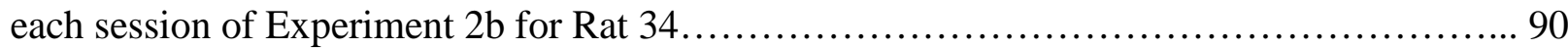




\section{Introduction}

Behavior is a joint product of its history of reinforcement and interactions with the current environment. Although the emphasis on functional relations between present behavior and current environmental contingencies has long been the cardinal area of research, a growing body of research is focused on the interaction between the behavioral history of the organism and present behavior. One such phenomenon is resurgence, the transient recurrence of a previously reinforced response following a worsening of reinforcement conditions associated with a current Alternative response (Lattal et al., 2017; Lattal \& St. Peter Pipkin, 2009; Shahan \& Craig, 2017). Resurgence differs from other types of extinction-induced variability because the response to be resurged typically has some explicitly arranged reinforcement history (i.e., that response has been explicitly reinforced in the past). Thus, resurgence can be conceptualized as a specific type of extinction-induced response variability generated by the interaction between the present environment and the behavioral history of the organism.

To distinguish resurgence from other instances of extinction-induced variability, a third operandum in which responding is never reinforced has been included (Epstein, 1983). Although this feature typically is described as a "control feature” (e.g., Podlesnik, JimenezGomez, \& Shahan, 2006; Sweeney \& Shahan, 2013) its presence raises some conceptual issues that warrant further investigation (Lattal, Solley, Cançado, \& Oliver, in press). The interpretation of Control-operandum responding is that it reflects the importance of reinforcement histories to resurgence in that in the absence of such history responding fails to develop on the other operandum. This interpretation, however, is restricted to contexts in which the behavioral history of the organism is explicitly controlled and known to the experimenter, as often is the case when experimentally naïve animals are used (e.g., Craig, Browning, Nall, 
Marshall, \& Shahan, 2017; Sweeney \& Shahan, 2013). A broader interpretation is that Controloperandum responding reflects the previous history of reinforcement for responding on that operandum and the relative recency of that reinforcement history in relation to that of the target response (Lattal et al., 2019). This latter interpretation could account for the absence of Controloperandum responding typically seen in nonhuman resurgence experiments. It also would account for the relative abundance of Control-operandum responding reported in human-operant resurgence experiments (e.g., Bolivar, Cox, Barlow, \& Dallery, 2017; Bruzek, Thompson, \& Peters, 2009; Sweeney \& Shahan, 2016).

Given the conflicting interpretations of Control-operandum responding, it remains an empirical question as to what variables affect such responding during tests of resurgence. Both extinction-induced variability and the organism's behavioral history have been suggested as possible sources of such Control-operandum responding; however, the roles of these variables have not been isolated. The proposed series of experiments will examine the interpretation of Control-operandum responding and how historical variables impact the resurgence of Controloperandum responding. In the literature review that follows, the determinants of resurgence are reviewed, as are variables that affect Control-operandum responding.

\section{Literature Review}

\section{Determinants of Resurgence}

Resurgence typically is assessed using a three-phase procedure. In the first, or Training phase, a Target response is reinforced according to a given reinforcement schedule. During the second, or Alternative-Reinforcement (AR) phase, the Target response is no longer reinforced and a second, Alternative response is reinforced. Finally, in the third, Resurgence-Test (RT) phase, both the Target and the Alternative response are extinguished. Typically, by withdrawing 
reinforcement of the Alternative response, there is a transient increase in the Target response above its level in the preceding Alternative-Reinforcement phase. This transient increase in the Target response defines resurgence.

There are a number of variations on the typical three-phase resurgence procedure. One is to train both the Target and Alternative response concurrently during the Training phase, rather than training the Target and Alternative responses sequentially in the first two phases (e.g., Oliver, Nighbor, \& Lattal, 2018). Elimination procedures for the Target response during the AR phase include the differential reinforcement of other behavior (DRO) (Alessandri, Lattal, \& Cançado,2015; Doughty, da Silva, \& Lattal, 2007; Mulick, Leitenberg, \& Rawson, 1976), positive punishment (Kestner, Redner, Watkins, \& Poling, 2015), and negative punishment (Okouchi, 2015). Some variations of the RT phase include a reduction in reinforcer magnitude (Craig et al, 2017; Oliver et al., 2018), a decrease in reinforcement rate (Lieving \& Lattal, 2003; Volkert, Lerman, Call, \& Trosclair-Lasserre, 2009), and an increase in the delay to reinforcement (Jarmolowicz \& Lattal, 2014). This research has shown, among other things, that variables in effect in each phase of the resurgence procedure differentially affect the probability and magnitude of resurgence during the RT phase. Furthermore, these manipulations have given rise to a rich array of data that have refined how resurgence is characterized and identified more precisely the conditions under which behavior recurs.

Despite the advances described above, little attention has been given to distinguishing resurgence from more generalized extinction-induced variability in the experimental analysis of resurgence. For example, if the design of a resurgence experiment includes only two responses, the Target and Alternative, then increases in the Target response during the RT phase may be difficult to interpret. The increase in the Target response could be due to either a previous 
reinforcement history or extinction-induced variability (e.g. Antonitis, 1951; Eckerman \& Lanson, 1969). Thus, it is necessary to include a procedure to isolate responding due to a reinforcement history from generalized increases in responding during extinction.

To distinguish resurgence from extinction-induced variability a third, Control operandum, sometimes is incorporated into the resurgence procedure. Epstein (1983) reported that when he used a Control operandum, Target responding exceeded Control responding during the RT phase. During the Training phase, key pecks to the Target response were reinforced and responses to a second, Control operandum, were recorded, but were without consequence. Once responding was stable, the Target response was extinguished and a second, arbitrarily selected, response (e.g., head bobbing, turning, etc.) was reinforced. Finally, during the resurgence test, extinction was in effect for both the initially trained response and the Alternative response. During the RT phase, the number of Target responses ranged from 25 to 575, whereas the number of Control responses for all six pigeons was one. Epstein argued that this result demonstrated that resurgence was distinct from extinction-induced variability and that resurgence was due to the explicit reinforcement history of the Target response. Consequently, the inclusion of a Control operandum has become the gold standard for separating resurgence from other extinction-induced responding. Less responding on this operandum during the resurgence test is interpreted as evidence that the recurrence of the Target response is due to the explicitly arranged reinforcement history and not a general increase in responding (e.g., Cleland, Foster, \& Temple, 2000; Craig et al., 2017; Sweeny \& Shahan, 2013).

To summarize, resurgence is the product of the interaction between the current environment and the reinforcement history of the organism. Although extinction is a generative process of behavior, resurgence differs from extinction-induced variability as it is due to a 
previous history reinforcement. Epstein (1983) demonstrated, through the inclusion of a response present but with no experimental reinforcement history (i.e., a Control response), that increases in responding during the RT phase were isolated to the response that was associated with a reinforcement history. The inclusion of the Control response has become the procedure to isolate resurgence from other extinction induced processes. Despite the conceptual and practical utility of including a Control response in resurgence procedure, its inclusion poses some conceptual challenges regarding its interpretation. The following section will discuss some of the issues with the Control response.

\section{The Control Response and its Implications}

Other interpretations than the above of Control-operandum responding also may be tenable. For example, the absence of Control responding may suggest that the response option is simply not a distinguishable part of the environment. It could, however, also truly reflect an absence of a history of reinforcement of Control responding. In situations in which the Target response lacks an explicit history of reinforcement, resurgence has not been found. da Silva, Maxwell, \& Lattal (2008, Experiment 1) omitted the Training phase of a resurgence procedure and only established a reinforcement history for the Alternative response. During the RT phase, responses to both the Alternative and Target were extinguished and an increase in the Target did not occur. Thus, in contexts in which the behavioral history of the organism has been strictly controlled (i.e., experiments conducted with non-human participants), the lack of Control responding may be due to a lack of a reinforcement history. This lack of reinforcement history, presumably, is a determining variable for why Control responding does not occur.

Despite the absence of an explicit reinforcement history there sometimes is minimal Control-operandum responding during the RT phase. Two notable exceptions are Epstein (1983) 
who reported only one Control response during the RT phase and Cleland et al. (2000) who reported including a Control operandum but failed to report whether Control responding occurred during the RT phase. Presumably, Control responding did not occur. In the cases in which Control responding is reported, however, it typically does not exceed Target responding in resurgence experiments in which nonhuman animals are the subjects (e.g., Craig et al., 2017; Sweeney \& Shahan, 2013).

One variable that may account for such modest increases in Control responding during the RT phase is the presence of reinforcer deliveries during the Training phase. Winterbauer and Bouton (2010; see also da Silva et al., 2008) investigated whether resurgence-like effects occur in the absence of explicitly reinforcing the target response in a Training phase. Three different Training phases were arranged, using a between-groups experimental design. During the Training phase, rats were divided into three groups: Resurgence, Free, and No Training. For the Resurgence group, Target responses were reinforced according to a random interval (RI) 30-s schedule. For a second, Free, group, Target responses did not produce any consequence, but response-independent food deliveries were yoked (i.e., a yoked Variable Time (VT) schedule) to match the reinforcement conditions of rats in the resurgence group. Finally, for a No Training group, there were no programmed consequences for pressing the Target lever, nor were there any response-independent reinforcer deliveries. Following an AR phase, in which an Alternative response was reinforced, reinforcement was eliminated during the RT phase. During the RT phase, there was more responding by rats in the group in which Target responses were reinforced during the Training condition. There also was, however, a small, but statistically significant increase in Target responding relative to the AR phase observed for both the Free and No Training groups. Although the amount of Target responding for the Free and No Training 
groups, was less than that of the Resurgence group, the increase in Target responding was interpreted to suggest that extinction-induced variability accounts for some of the responding observed during the RT phase. Alternatively, the delivery of response-independent reinforcers may facilitate some responding during the Training and as a result may account for the resurgence-like effects observed with the Free Group. Winterbauer and Bouton's results taken together with da Silva et al. (2008) indicate that a previous history of reinforcement is necessary for resurgence, however, increases in the Control response could reflect in part extinctioninduced variability precipitated by the withdrawal of reinforcement. Relative to the Target response, however, Control responding is of lesser magnitude and occurs inconsistently relative to target responding (e.g., Epstein, 1983).

Although Control responding occurs inconsistently with nonhuman subjects in resurgence experiments, in related experiments with human subjects, the amount of Controloperandum responding during the RT phase has been reported to equal or exceed Target-key responding (e.g., Bolivar et al., 2017; Bruzek et al., 2009; Sweeney \& Shahan, 2016). Sweeney \& Shahan (2016) assessed resurgence using a discrete-trial procedure, in which participants were required to click on shapes presented on a computer screen. During each trial participants were presented with three shapes (i.e., Target, Alternative, and Control) and points were awarded for correct responses. Correct responses depended on which phase was in effect, with correct responses to the Target and Alternative shapes resulted in points in their respective phases. During the RT phase, in which no points were delivered for selections, responding was undifferentiated across the Target, Alternative, and Control responses. Although the Target response resurged, it was accompanied by Control-response increases. Sweeny and Shahan concluded that the latter increase made it difficult to attribute the increase in the Target response 
to a previous history of reinforcement.

The issue of Control responding is not limited to the discrete-trial procedure used by Sweeney and Shahan (2016). Bolivar et al., (2017), used a free-operant procedure to examine resurgence using human participants. Participants clicked objects on a computer screen for points. A three-phase resurgence procedure was used, in which responding to the Target and Alternative, were reinforced in their respective phases. Similar to Sweeney and Shahan, Control responding was similar to or exceeded the number of Target responses during the RT phase. To determine whether resurgence could be obtained, a number of procedural variations were investigated. The only condition in which resurgence occurred without a corresponding increase in Control responding was when the Target and Alternative responses were presented in their corresponding phases (i.e., Training and AR phase) and the stimulus conditions present during the Training condition were reintroduced during the RT phase (i.e., a superimposed renewal procedure). Bolivar et al. argued that although resurgence occurred during a condition in which the response options were introduced in each phase and accompanied by stimuli associated with each phase, this was not reflective of a typical of a resurgence procedure (see, however, Kincaid, Lattal \& Spence, 2015, for a similar procedure they termed Super Resurgence). The implication of the results reported by Sweeney and Shahan and Bolivar et al., is that Control responding reflects extinction-induced response variability, thus making it difficult to discern resurgence from extinction-induced variability in human-operant experiments on resurgence.

Although the procedures to which participants were exposed were "novel," in the sense that the participants did not have previous experience with the experimental task, they were not truly novel, as human participants presumably have extensive experience clicking objects on computer screens. Thus, an alternative interpretation is that Control responding in human- 
operant resurgence experiments simply reflects the pre-experimental learning history related to key pressing of the individual. This interpretation is supported by the results of Bruzek et al. (2009), who assessed the resurgence of infant caregiving response. Each session, the experimenter recorded the occurrence of three behaviors (e.g., playing, rocking, or feeding), the Target, Alternative, and Control response. During the Training phase of the procedure, a given response was reinforced by terminating the crying of a baby doll. Next, that response was extinguished and another response was trained. When both the Target and Alternative responses no longer terminated crying (i.e., extinction) the initially-trained response recurred. For one participant, however, there was a corresponding increase in the Control response (i.e., feeding)

during the RT phase. Bruzek et al. suggested that the increase of the Control response may have been due to the participant's extensive prior history of caregiving. Thus, the temporally distant reinforcement history of the organism provided for Bruzek et al. a plausible explanation for observed Control responding during a resurgence experiment.

\section{Resurgence of Multiple Responses: Implications for Understanding Control Responding}

If Control responding during a resurgence test is conceptualized as an instance of resurgence of a response of extra-experimental reinforcement history, then the relative resurgence of two responses can be experimentally assessed. Of the relatively few experiments that have assessed the resurgence of multiple responses there have been two conflicting findings. The first is termed a primacy effect which refers to the tendency of an initially reinforced response to resurge first and with a greater magnitude than a more recently reinforced response (Reed \& Morgan, 2006). Conversely, a recency effect refers to the tendency of a more recently reinforced response to resurge more vigorously and earlier than more distally reinforced response. Given the relative paucity of research on the resurgence of multiple responses, it is not 
clear as to the determining factors that affect resurgence under these conditions.

Reed and Morgan (2006) were one of the first to report that initially reinforced responses were more likely to resurge than more recently reinforced responses. Using a three-phase procedure, response sequences were reinforced until the sequence occurred correctly greater than $80 \%$ of trails for three consecutive sessions. Over the course of the procedure, three response sequences were reinforced. Following the training of the third sequence, an extinction phase was in effect for two consecutive sessions. During the first extinction session, resurgence of the first trained sequence was observed for all six rats, however, resurgence of the second trained response was observed for five of the six rats. Furthermore, there were more instances of the second trained sequence for three rats during the first extinction session. Unlike the first extinction session, during the second session, the resurgence of the second trained sequence was greater than that of It should be noted, however, that resurgence of the first trained sequence resurgence of the first trained sequence was observed during the second extinction session. Although Reed and Morgan argue that the results reported indicate a primacy effect, the fact is that results were inconsistent and resurgence of the second trained sequence exceeded the resurgence of the first trained sequence for all rats.

More robust evidence of a primacy effect was reported by Bruzek et al. (2009; Experiment 2). In this experiment, the relative resurgence of two different responses that differed not only by the order of the reinforcement history of the response, but the total duration each response was reinforced. In this experiment, the reinforcement contingency for the first trained response was in effect until the response had occurred continuously for five minutes for three consecutive sessions. In the next phase, the initially reinforced response (i.e., Target 1) was no longer reinforced and a second response was reinforced. This phase was in effect until 
the response had occurred for five minutes for two sessions. Following these two training phases, an extinction session followed by an alternative reinforcement and resurgence test session was conducted. During the resurgence test phase, Target 1 resurged more vigorously than Target 2 for all three participants. Bruzek et al. argue that the greater resurgence of the initially trained Target was due to the relatively longer reinforcement history.

Despite the evidence of a primacy effect provided by Reed and Morgan (2006) and Bruzek et al. (2009) there are several critical confounds that undermine the primacy account of resurgence. First, both Reed and Morgan and Bruzek et al. set up unequal training times for each of the responses. Thus, it is possible, that responses with more extensive reinforcement histories may resurge more robustly even when there are more recently reinforced responses.

Furthermore, each of the resurgence test phases were relatively brief, Reed and Morgan had only two sessions and Bruzek et al. only had one. Lattal, et al., (in press) investigated the role of reinforcement histories on Control-key responding with pigeons. Explicit reinforcement histories were established for what might be considered Control-key responding during a first phase. In a second phase, in what conventionally would be considered the Training phase, responses to a second key, or Target response, were reinforced according to a similar schedule of reinforcement and Control-key responses were no longer reinforced. During the next, AR phase, responses on the Target key were extinguished and responses to a third, Alternative Response key were reinforced. Finally, during the RT phase, extinction for all keys was in effect. Resurgence of both the Target and Control responses during the RT phase occurred. There was, however, less responding on the Control than on the Target key and responding on the Control key resurged later than did that on the Target key. Thus, under the conditions specified, more recently reinforced responses resurged sooner than those reinforced earlier (cf. Reed \& Morgan, 
2006). Although Control-key (first-trained) responding was less than that of the Target, Lattal et al.’s findings are notable nonetheless, because they suggest that Control-key responding may reflect a temporally distant reinforcement history and not extinction-induced variability.

The suggestion that Control responding may reflect a more distal reinforcement history than the one explicitly arranged in an experiment is conceptually and methodological important in two ways. First, accounting for Control responding as an artifact of a previous reinforcement history allows resurgence to be delineated from extinction-induced variability (see also, Lattal et al., in press). Second, if Control responding is indeed a function of a previous history of reinforcement, then it should be sensitive to the same kinds of contingencies that affect the recurrence of the Target response. For instance, da Silva et al. (2008) reported that the absolute magnitude of resurgence was functionally related to the response rate of the Target response during the Training phase. During the Training phase, pecking either of two response keys were reinforced according to a concurrent VI 1-min VI 6-min schedule. Following this phase, responding to both keys was no longer reinforced and responding to an Alternative, third key, was reinforced according to a VI 3-min schedule of reinforcement. Finally, during the RT phase, extinction was in effect for all three keys. In absolute terms, the resurgence of the Target response previously maintained by the richer schedule of reinforcement was greater than the response previously maintained by the leaner schedule. Even when reinforcement rates are held constant, but response rates differ, the magnitude of the resurgence effect can be mediated by the response rate of the Target during the Training phase (da Silva et al., Exp. 3). Presumably variables such as response and reinforcement rate also should affect the extent of Controloperandum responding. 


\section{Statement of the Problem}

Although resurgent behavior is conceptualized as the recurrence of a previously reinforced response rather than an instance of extinction-induced variability, this delineation of processes is not entirely clear. This problem is exacerbated when the resurgence procedure involves only a Target and Alternative response, as resurgence of the Target could be a product of extinction-induced variability. Epstein (1983) introduced a potentially important methodological feature to address this issue: The Control response. Responding on a Control operandum, which is never reinforced, in a resurgence procedure has been interpreted to be extinction-induced variability (Bolivar et al., 2017; Epstein, 1983; Sweeney \& Shahan, 2016), however, this interpretation raises questions because either the presence or absence of Control responding may indicate extinction-induced variability. Within the operant chamber there is an almost infinite variety of responses that might occur, and extinction might increase any one or any combination of them, thus there is no reason to think that a control response for which there is no history of reinforcement will somehow "attract" induced responses over some other behavioral form. It may be the case that, however, that some Control responding may occur as an instance of response generalization due to the similarity of the Control and Target operanda. Conversely, the absence of responding on the Control operandum truly may be due to the absence of a reinforcement history for the Control response, however, given that almost any class of behavior may be evoked by extinction, this conclusion is tenuous as well. Therefore, to be able to interpret the meaning of Control responding, it is necessary to identify the conditions under which Control responding occurs. As Bruzek et al. (2009) and Lattal et al. (in press) have noted, rather than being extinction induced, one such source of Control responding may be the history of reinforcement of that response. If Control responding is a manifestation of behavioral 
history, then such responding is just another resurgence effect, albeit moderated by the more distal reinforcement history of that response.

Thus, it is an empirical question as to what variables affect the occurrence of Control responding during the RT phase. Lattal et al. (in press) found that what could be considered Control-operandum responding was related to a previous history of reinforcement, however, the relatively weak resurgence of Control-operandum responding was because of the competition from the resurgence of a more recently reinforced response (c.f. Reed \& Morgan, 2006). Despite the relatively low amounts of resurgence reported by Lattal et al., there are any number of variables other than extinction-induced variability that may impact Control-operandum responding during a resurgence test. For example, da Silva et al. (2008) reported response rate during the Training phase was systematically related to the magnitude of the resurgence effect. These variables impact the resurgence of the Target response during the RT phase; however, they have not been assessed in the context of their potential effects on Control-operandum responding.

If Control responding is a function of a previous reinforcement history as suggested by Bruzek et al. (2009) and Lattal et al. (in press) then the probability of such responding during the RT phase should be related to changes in both proximal and distal historical events. Therefore, the purpose of the proposed experiments was to analyze variables affecting Control-operandum responding during the RT phase of a resurgence procedure. The first experiment assessed how Control responding may be evoked as a function of how the Target response is maintained during the Training phase. The second was a systematic replication and extension of Experiment 1 , in which effects of a previous reinforcement history for Control responding on the resurgence of Control responding were assessed. Finally, Experiment 3 examined the relation between 
different histories of reinforcement of response rates and the subsequent resurgence of Control responding.

\section{General Method}

\section{Overview}

In the experiments reported below, the resurgence of operant behavior was assessed using variations of a prototypical resurgence procedure (cf. Lieving \& Lattal, 2003). The procedure of each experiment consisted of at least one Training phase, an AR phase, and a RT phase. The following methods were common to Experiments 1, 2a, and 3. This includes the subjects, apparatus, and the AR and RT phases.

\section{Subjects}

Pigeons. Male White Carneau pigeons were used. Each was maintained at $80 \%$ of its ad libitum body weight (+/- 15g) by postsession feeding as required. When not in the operant chambers, the pigeons were caged individually in a vivarium with a 12:12-hr light/dark cycle and with continuous access to water and health grit in their home cages. The presence or absence of an experimental history for each pigeon is stated in this section of each experiment.

\section{Apparatus}

Four standard operant chambers were used in these experiments. Each chamber had three walls that were made of plywood and a fourth wall (work panel) made of aluminum. The latter wall contained three 2-cm diameter Gerbrands Co. response keys arranged in a row, spaced $7 \mathrm{~cm}$ apart, center to center, with those centers located $20 \mathrm{~cm}$ from the floor. Keys were transilluminated by 28-v DC light bulbs with colored translucent covers. The specific colors are noted in the apparatus section of each experiment. A standard food hopper was located on the work panel, behind a $6 \mathrm{~cm} \mathrm{~W} \mathrm{x} 5 \mathrm{~cm} \mathrm{H}$ rectangular aperture, $13.5 \mathrm{~cm}$ from the door and with its 
lower edge $9 \mathrm{~cm}$ from the floor. During reinforcer deliveries, the aperture was illuminated by a 7-W, 28-V bulb and the hopper was raised, allowing the pigeon 3-s access to Purina NutriBlend ${ }^{\mathrm{TM}}$ pellets. A 7-W, 28-V bulb, located behind a 4-cm square aperture located $24 \mathrm{~cm}$ from the door with its lower edge $4 \mathrm{~cm}$ from the floor, provided general illumination. The pigeon chambers were controlled with Dell ${ }^{\circledR}$ computers running MED PC IV® software in an adjacent

room. A fan located on the outside of each chamber provided ventilation. The rooms housing the chambers also contained a white-noise generator to mask extraneous sound.

\section{Procedure}

Sessions occurred 7 days a week at approximately the same time each day. Each session began with a 3-min blackout of the chamber. Following the blackout, all response keys and the houselight were activated. In all experiments, responses to all response keys were recorded. A 3-s response-response change-over delay (COD; Shahan \& Lattal, 1998) was in effect on each key throughout all phases except the RT phase. This COD was programmed such that the first response on the changed-to key initiated a 3-s interval after which subsequent responses on that key were eligible for reinforcement.

Preliminary Training. All naïve animals first were magazine trained. Following magazine training, key-peck responses were established by a combination of differential reinforcement of successive approximations and autoshaping (Brown \& Jenkins, 1968). Responses then were reinforced on a fixed-ratio (FR) 1 schedule that subsequently was leaned to the terminal schedule value for the Target and Alternative responses.

Alternative Reinforcement (AR) Phase. During this phase, a VI 60-s schedule was in effect for the Alternative Response. The VI schedule was composed of 11 intervals generated using Flesher and Hoffman’s (1962) distribution. The intervals were selected randomly and 
without replacement. Responses to the other keys were recorded, but extinction was in effect. Sessions terminated once 60 reinforcers were delivered or 75 min had elapsed. This phase continued for at least 10 sessions and until Target responding was less than 1 response per minute (rpm) for three consecutive sessions. Deviations from this procedure are described in each experiment.

Resurgence Test (RT) Phase. Extinction was in effect for all response keys. Session durations were yoked to the average session duration of the last three sessions of the preceding phase. This phase was in effect for at least seven sessions and until responding on each key was less than $1 \mathrm{rpm}$ for three consecutive sessions. Any deviations from this procedure are described in each experiment.

\section{Experiment 1}

In a typical resurgence procedure, the reinforcement history established during the Training phase generally obscures the recurrence of responses with extra-experimental reinforcement histories. This effect, however, only may be the case when the to-be resurged response is a discrete response such as a key peck or lever press. If Target responses postpone reinforcement, as in the case of a DRO contingency, then it would follow that during the RT phase "not responding" would be the resurgent response class and one would observe little to no responding on the Target operandum. Under these conditions, it is possible that the resurgence of behavior with an extra-experimental reinforcement history may manifest during a resurgence test along the lines described by Lattal et al. (in press). Therefore, the purpose of Experiment 1 was to assess the effects of a DRO history for the Target response on its recurrence during the RT phase of the Target-response as well as behavior with a pre-experimental reinforcement history. 


\section{Method}

\section{Subjects}

Four male White Carneau pigeons as described in the General Method section were used. Each had a history of keypecking on various schedules of reinforcement.

\section{Apparatus}

Two operant chambers as described in the general method section were used.

\section{Procedure}

Figure 1 is a diagram of the procedures in effect during each phase of Experiment 1 . The locations of the Target and Control keys were counterbalanced across pigeons and all keys were transilluminated during all sessions, except during reinforcement, and across phases. The response-key assignments for each pigeon are shown in Table 1.

Training Phase. During the Training phase, two independent schedules of reinforcement were in effect. Pecking the Alternative key was reinforced according to a VI 60-s schedule. Concurrent with the VI schedule, reinforcers were delivered according to a tandem variable-time (VT) 50-s variable DRO (VDRO) 10-s schedule. The VT schedule contained 11 intervals generated as described above. During the VT component of the tandem schedule, responses were recorded, but were without programmed consequence. Once the VT interval elapsed, the VDRO component was initiated. In the VDRO component, each key peck on any of the response keys reset the DRO timer. The VDRO schedule contained 10 intervals, ranging from 0.5 to 33 s, generated using Flesher and Hoffman’s (1962) distribution. When the DRO timer elapsed, a reinforcer was delivered and the VT component was reinstated. Sessions terminated after 60 reinforcers. Reinforcer deliveries on the VTVDRO also were separated by at least $3 \mathrm{~s}$ from responses to either the Alternative or Control keys. Responses to the Control key 
were recorded but were without programmed consequences. This condition was in effect for at least 13 sessions and until Target response rates were less than 1 response per minute for three consecutive sessions.

AR Phase. This phase was as described in the General Method section.

RT Phase. This phase was as described in the General Method section.

\section{Results}

Figure 2 shows responses per minute for the Target, Control, and Alternative responses (hereafter, Target, Control, and Alternative) for each pigeon during each phase of the experiment. During the Training phase, response rates for the Target and Control keys of each were at or near zero levels. Alternative-response rates for individual pigeons ranged from approximately 30 rpm for Pigeon 847 to nearly 90 rpm for Pigeon 267. When the DRO was suspended in the AR phase, Alternative-response rates of each pigeon increased, but these increases were more modest for Pigeon 847. Target and Control response rates remained zero throughout the AR phase.

Implementing extinction during the RT phase rapidly diminished Alternative response rates for all pigeons. These rates decreased to zero within two sessions for Pigeon 847 and four for the other three pigeons. Figure 3 shows, on an enlarged Y-axis scale, only Control and Target response rates for the last three sessions of the AR phase and the RT phase. Control response rates increased from zero at the end of the AR phase to a high of 15 rpm for Pigeon 567 and 7 rpm for Pigeon 267 during the RT phase. Target response rates increased relative to the last three sessions of the AR phase, during the first two to three sessions for Pigeons 567 and 267, however Target response rates did not exceed Control-response rates during this period. For Pigeon 267 Target response rates increased between Sessions 6 and 12 before returning to 
less than $1 \mathrm{rpm}$. Negligible increases in Control and Target response rates occurred for Pigeons 9553 and 847.

\section{Discussion}

In a typical resurgence procedure, the Target response is a discrete measured response (e.g., a key peck or a lever press). Here, however, the Target response was the absence of recorded key pecks. Thus, during the RT phase, the to-be resurged response was nonengagement with the Target operandum. Under these conditions it would be expected that Target-operandum responding would not increase. This was the case for Pigeons 847 and 9553. For these pigeons, Target responding was similar to the Control response, a response that lacked an intraexperimental reinforcement history. For Pigeons 267 and 567, however Control-response rates exceed Target-response rates during the first several sessions of the RT phase. In the conventional account of resurgence, such responding typically is described as extinction-induced variability (e.g., Epstein, 1983; Sweeney \& Shahan, 2016).

The results of the RT are difficult to interpret in relation to the proximal reinforcement history of the Target response (i.e., nonengagement) and the unknown pre-experimental reinforcement history of each of the pigeons used in the experiment. The absence of key pecking for Pigeons 847 and 9553 could be interpreted as the resurgence of nonresponding because the absence of a discrete response (e.g., key peck) was the Target response. Similarly, the absence of Control responding also might be an instance of negative induction from the DRO-defined target response. Thus, for Pigeons 847 and 9553 it is possible that the same behavioral process controlling the nonengagement with the Target response also controls the lack of Control responding during the RT phase.

The present results highlight an issue in interpreting Control responding during an RT 
phase. For Pigeons 267 and 567 it is not clear whether the increases in Control-response rates were due to a prior, distal, history of key-peck reinforcement or were an instance of extinctioninduced variability. A case can be made that the increases in Control-response rates represents the resurgence of a response established during a prior reinforcement history. For these two pigeons, the increases in Control-response rates were transiently persistent in that they remained higher relative to the Target-response rates for four to five RT phase sessions before dissipating. This transient increase in response rates was followed by a decline, which is characteristic of a resurgence effect (Lattal et al., 2017). Conversely, Control-response rate increases did not occur for the other two pigeons. This latter result may be due to the absence of an intra-experimental reinforcement history for the Control response. There are two other possible interpretations of the absence of Control responses for Pigeons 847 and 9553. One is that the DRO contingency in effect for the Target key during the Training and AR phases also exerted some control over Control responding. The other is that the Control key was simply an irrelevant feature of the environment, no different from any other feature that fails to control responding.

The outcome of an RT phase typically is assessed as a function of the variables manipulated within each preceding phase of the resurgence procedure. A limitation of interpreting the outcome of the RT phase based on the contingencies within each phase of the resurgence procedure is that the previous reinforcement history of the organism is not considered. In the present experiment, each of the pigeons had a previous history of responding on various schedules of reinforcement. Thus, the results cannot be interpreted unequivocally.

\section{Experiment 2}

To further address the question of the role of previous experiences in resurgence, Experiment 2 was conducted to further examine the relation between relatively proximal and 
relatively distal reinforcement histories and resurgence using both a conventional 3-phase acrosssession resurgence procedure (Experiment 2a; cf. Epstein, 1983) and a within-session resurgence procedure (Experiment 2b; cf. Cook \& Lattal, 2019). A secondary purpose of Experiments 2a and $2 \mathrm{~b}$ was to address the possibility that Control responding may be due to response generalization by comparing the resurgence of a distally reinforced response (i.e., Target 1) and a response that lacked a reinforcement history (i.e., a Control response).

\section{Experiment 2a}

\section{Subjects}

Three experimentally naïve male White Carneau pigeons were used. Each was maintained as described in the General Method section.

\section{Apparatus}

The operant chamber was as described in the General Method section except for the addition of a metal panel on the back wall (hereafter the rear panel). The rear panel contained a single 2-cm diameter Gerbrands Co. response key located on the panel midline, $9 \mathrm{~cm}$ from the ceiling. The key was transilluminated (color) by 28-V bulb at all times except during reinforcement.

\section{Procedure}

Figure 4 shows a diagram of the procedure. Each pigeon was exposed to the phases described below in the order in which they appear. Following the presession blackout, all four keys and the houselight were activated. The locations of the Target 1 and Control keys on the front work panel were counterbalanced across pigeons and all keys were transilluminated at all times except during reinforcement during all sessions across phases. The key assignments for each pigeon are in Table 2. 
Target-1 Training phase. The first, or Target 1, response was reinforced according to a VR 30 schedule of reinforcement. The VR response requirement ranged from 2-101 responses and was generated using the Flesher and Hoffman (1962) distribution. The ratio values were selected randomly and without replacement. This phase was in effect for at least 10 sessions and until Target 1-response rates were either stable or showing an increasing trend, based on visual inspection.

Target-2 Training phase. The same tandem VT 50-s VDRO 10-s schedule of reinforcement described in Experiment 1 was in effect for Target-2 (center) key pecks. Extinction was in effect for all other responses. This condition was in effect for at least 10 sessions and until the Target 1-response rate was below $1 \mathrm{rpm}$ for three consecutive sessions.

AR phase. This phase was as described in the General Method section.

RT phase. This phase was as described in the General Method section.

\section{Results}

Figure 5 shows Alternative, Control, Target-1, and Target-2 response rates for each pigeon across each phase. By the end of the Target- 1 training phase, Target- 1 response rates were above $120 \mathrm{rpm}$ for each pigeon. Alternative, Control, and Target 2-response rates were near zero during this phase. During the Target-2 training phase, Target 1-response rates decreased for each pigeon. Target-1 and Alternative-response rates of Pigeons 18623 and 35424 increased to approximately $1 \mathrm{rpm}$ for one to two sessions during this phase. By the end of the Target-2 training phase, however, there was no responding on any of the keys. During the transition to the AR phase, transitory increases in Target-1 response rates occurred all three pigeons. Following this transitory increase, Target-1 response rates returned to zero levels. Alternative-response rates were maintained at moderate to high response rates for Pigeons 18263 
and 35424, whereas Alternative-response rates for Pigeon 18057 were more variable and lower. When extinction was implemented during the RT phase, Alternative-response rates declined for each pigeon. Target-1 and Control response rates increased for Pigeons 18057 and 35424. For Pigeon 18623, however, Control, Target-1, and Target-2 response rates increased. Relative to Pigeons 18057 and 18263, the increases in Target-1 and Control-response rates for Pigeon 35424 were more substantial. The Left graphs of Figure 6 show, on an enlarged y-axis scale, Control, Target-1, and Target-2 response rates during the last three AR-phase sessions and each RT-phase session. For Pigeon 18057, Target-1 response rates increased from 0 to 0.5 rpm. Except during Session 2, Target-1 response rates were higher than those of the Target-2 or Control responses. Control response rates were consistently higher than Target-1 and Target-2 response rates for Pigeon 18623 during the first two sessions of the RT phase. Substantial increases in Target-1 response rates occurred for Pigeon 35424 during the first session of the RT phase. Control response rates also increased and were higher than Target-1 response rates during the second session of the RT phase for this pigeon. The right graphs of Figure 6 show the total number of responses on the Target-1, Target-2, and Control keys during the last three sessions of the AR phase and the first three sessions of the RT phase. For Pigeons 18057 and 35424, there were more Target- 1 responses than Target-2 and Control responses during the first three sessions of the RT phase. There were more Control responses than Target-1 and Target-2 responses for Pigeon 18263.

\section{Experiment 2b}

This experiment was a systematic replication of Experiment 2a, with two important changes. First, the procedure was adapted from the within-session resurgence procedure described by Cook \& Lattal (2019). Thus, each session consisted of all the phases of the 
resurgence procedure. Second, rats rather than pigeons served as subjects.

\section{Subjects}

Four experimentally naïve male Sprague-Dawley rats, approximately 6-months old at the beginning of Experiment 2b were used. Each was food restricted for 22 hours prior to the start of each session. The rats were pair-housed with free access to water in a vivarium under a 12-hr light: 12-hr dark cycle. Sessions occurred during the dark cycle.

\section{Apparatus}

A single sound-attenuated operant chamber was used. It had a work area of $29.53 \mathrm{~cm} \mathrm{~L}$ by $24.84 \mathrm{~cm} \mathrm{~W}$ by $18.67 \mathrm{~cm} \mathrm{H}$. Two of the walls were of clear plastic and the other two were aluminum. On one wall, hereafter, the front panel, there were three MED Associates ${ }^{\circledR}$ model no. ENV-116RM retractable levers and cue lights. The center of each lever was located $5 \mathrm{~cm}$ from the floor and the levers were $7 \mathrm{~cm}$ apart, center to center, from each other. The cue lights were approximately $6 \mathrm{~cm}$ above the center of each lever. On the opposite aluminum wall, hereafter, the rear panel, there was a food magazine aperture and a Med Associates® model no. ENV-116RM retractable lever. A Med Associates ${ }^{\circledR}$ model ENV-203M-45 pellet dispenser, located behind the rear panel, delivered BioServ ${ }^{\circledR} 45$ mg Dustless Precision Pellets ${ }^{\circledR}$. To the right of the food magazine and in the center of the rear panel, a Med Associates ${ }^{\circledR}$ retractable lever and cue light were $12 \mathrm{~cm}$ from the nearest clear plastic wall. A houselight was located at the top center of the rear panel, $12 \mathrm{~cm}$ from either of the plexiglass walls and $16 \mathrm{~cm}$ from the floor. To the right of the rear lever and adjacent to the clear-plastic wall a speaker provided white noise. A relay in the same location provided auditory feedback during reinforcer deliveries. 


\section{Procedure}

Figure 7 shows a diagram of the procedure. Each session began with a 3-min blackout, after which the houselight and cue lights were illuminated. All four levers were inserted into the chamber at this time and remained in these locations until the session ended. The procedures described below were replicated over five consecutive sessions. During each session, each of the following phases were in effect for the durations noted and sessions terminated following the RT phase of the procedure. Thus, each session comprised a complete 4-phase resurgence testing procedure.

Target-1 Training phase. Responses on the Target-1 lever were reinforced according to a VR 15 schedule. Responses on the other three concurrently available levers were recorded but had no programmed consequences. This phase was in effect until 10 reinforcers were delivered and no more than five responses occurred on the other levers across the last five reinforcer deliveries.

Target-2 Training phase. Responses to the Target-1, Control, and Alternative levers were recorded, but without programmed consequences. A tandem VT 20-s VDRO 10-s was in effect on the Target-2 lever. Responses to the Target-2 lever during the VT component were recorded, but without programmed consequences. When the VDRO 10-s schedule was in effect, Target-2 lever presses reset the DRO interval. The VDRO intervals ranged from $0.5 \mathrm{~s}$ to $33 \mathrm{~s}$. Once a reinforcer delivery occurred, the VT component of tandem schedule was reinstated. A COD was in effect in this and the next phase such that reinforcer deliveries were separated by at least $3 \mathrm{~s}$ from a response to either of the other levers. This phase was in effect until 10 reinforcers were delivered and no more than five responses occurred on the other levers across the last five reinforcer deliveries. 
AR phase. Alternative-response lever presses were reinforced according to a VI 30-s schedule. Responses on the other levers were recorded, however, they were without programmed consequences. This phase was in effect until 10 reinforcers were delivered and no more than five responses occurred on the other levers across the last five reinforcer deliveries.

RT phase. Extinction was in effect on each lever. This phase was in effect for 30-min.

\section{Results}

Figure 8 shows the number of Target-1, Target-2, and Control responses during the RT phase for each session. The number of Target-1 responses exceeded the number of Target- 2 and Control responses for each rat across each RT phase. For Rats 31 and 33 there were more Target-1 responses than Target-2, Alternative, and Control responses during the first RT phases, followed by a decrease in subsequent RT phases. Target-1 responding was more variable for Rats 32 and 34. There was no systematic difference in the number of Target-2 and Control responses. During the first RT phase, Rat 32's Target-2 and Target-1 responses were similar, For Rat 34, the most Target-2 responses occurred during the fifth RT phase. Cumulative records of lever pressing during each phase of each session for all rats are in Appendix B. During each phase, responding reflected the contingencies in effect. Target-2 and Alternative responses, however, did occur during the Target-2 training phase for all rats.

To analyze the temporal distribution of responding during each RT phase for each session heatmaps of Target-1, Target-2, and Control responding were constructed and are shown in Figure 9. Each box (i.e., 1-min bin) shows the frequency of responses such that darker boxes indicate a higher number of responses. By displaying the data in this way, the frequency of responding as a function of session time can be shown across successive RT phases. For the Target-1 response, the number of 1-min bins in which a response occurred decreased across 
successive resurgence tests. Furthermore, the frequency of Target-1 responding per each 1-min bin also decreased across successive RT phases. For each rat, Target-1 responding tended to occur within the first 10 min of each RT session. Target-2 and Control responding was more widely distributed across each bin in each RT phase. That is, unlike Target-1 responding, Target-2 and Control responding was less frequent and occurred during a fewer number of 1-bins per RT phase.

\section{Discussion}

Experiment 2 was designed to evaluate the resurgence of a response with a more distal reinforcement history (i.e., Target 1) relative to a response with a more proximal reinforcement history of not engaging in the Target response (Target 2). This was done by arranging a reinforcement history for a response (i.e., Target 1) and then systematically replicating the procedure used in Experiment 1. Thus, this experiment was a systematic replication of Experiment 1 with an added Training phase before the replication of the Experiment 1 procedure. An additional feature of Experiments $2 \mathrm{a}$ and $2 \mathrm{~b}$, was that a fourth response for which no reinforcement history was established served as a true Control response. Thus, by comparing Target-1 and Control responding during the RT phase, the resurgence of a response with a distal reinforcement history (e.g. a model of a Control response with a distal reinforcement history) could be compared to that of a response lacking a reinforcement history (i.e., a true Control). For two of three pigeons in Experiment 2a, Target-1 responding exceeded both Target-2 and Control responding during the RT phase. In Experiment 2b, Target-1 responding exceeded both Target-2 and Control responding during each RT phase for all four rats. Taken together, the results of Experiment 2a and $2 \mathrm{~b}$ do provide mixed evidence that more distally reinforced responses resurge and that such responding generally exceeds the amount of responding 
allocated to a response that lacks a reinforcement history. Furthermore, Target-2 responding generally did not occur across both Experiments 2a and 2b.

The present experiment can be also interpreted in relation to the serial order in which a response is trained. Both Experiments 2a and $2 \mathrm{~b}$ provide further evidence supporting the notion that responses with a distal reinforcement history may resurge during a RT phase (cf. Bruzek et al. 2009; Lambert, Bloom, \& Samaha, 2017; Reed \& Morgan, 2006). Furthermore, the resurgence of a response with a more distal reinforcement history exceeds responding on a response without an explicit reinforcement history. Thus, if Control responding is observed in a three-phase procedure, then one source of such responding is its distal reinforcement history. It should be noted, however, that some response generalization occurred. That is, in both Experiments $2 \mathrm{a}$ and $2 \mathrm{~b}$, some responding was allocated to the response without a reinforcement history (i.e., the Control response in the present experiment). Such responding, however, was generally less than that allocated to the more distally reinforced response (i.e., Target 1), which in the present experiment modeled a Control response with a distal reinforcement history. The relative difference in Target 1-responding versus Control responding in the present experiment suggests that if substantial Control responding is observed in resurgence procedure, then a likely source of such responding may be a previous reinforcement history rather than an instance of extinction-induced variability.

Aside from demonstrating that responses with more distal reinforcement histories may resurge, the current experiments shed some light on the importance of more recent reinforcement histories. Across both Experiments $2 \mathrm{a}$ and $2 \mathrm{~b}$, Target-2 responding during the RT phase was consistently lower than Target-1 responding. Furthermore, Target-2 responding was less than Control responding, which suggests that the recent reinforcement history of the tandem 
VTVDRO contingency manifested during the RT phase. The lack of Target-2 responding during the RT phase also suggests the importance of the contingencies in effect while a response is maintained. When a response is not required for reinforcement, then increases in that response are generally not observed when other sources of reinforcement are suspended. Although it is possible that the absence of responding, as observed in the current experiment, may constitute resurgence, this conclusion is not tenable given the methods used in the current experiment. When a response is required, such as in the case of behavior maintained by a VR schedule, then it likely that such a response may resurge.

In Experiment 2a, however, the resurgence of the Target-1 response was not as substantial as what occurred in Experiment 2b. Two variables may contribute to difference the in the magnitude of Target-1 resurgence in Experiments 2a and 2b. One is the length of the Target-2 Training and AR phases prior to the RT phase in Experiment 2a (see also Sweeney \& Shahan, 2013; but cf. Lieving \& Lattal, 2003). The other is the way that the Target-2 training phase was arranged. In the present experiments, the pigeons and rats were presented with four responses during sessions. Under these conditions, the VTVDRO contingency may not be as salient as a contingency that requires a discrete response (e.g., a key peck or lever press). That is, nonengagement with all available operanda becomes the response-reinforcer relation rather than just for the Target-2 response. Resurgence can and does occur when a response is suppressed using a DRO contingency (e.g., Alessandri et al., 2015; Doughty, da Silva, \& Lattal 2007). In the case of Alessandri et al. (2015) and Doughty \& Lattal (2007), the RT phase occurred immediately following the DRO Alternative-reinforcement phase, whereas in the current experiment the AR phase followed the Target-2-Training phase. Thus, the combination of exposure to the VTVRDO contingency and extinction of the Target-1 response during the AR 
phase may be implicated in accounting for the results of Experiment 2a. Given the difference in the magnitude of resurgence obtained in Experiment 2b versus that obtained Experiment 2a suggests that it may have been a combination of these factors described above and the relative duration between the Target-1 response training phase and the RT phase.

The results of Experiment 2b extend the results of Cook and Lattal (2019) by demonstrating that the within-session resurgence procedure can be used to evaluate the resurgence of multiple responses. Similar to their results, the resurgence of Target 1 tended to be the highest during the first RT phase and then gradually diminished in magnitude for two of the four rats. Additionally, there were limited differences in Control and Target-2 responding across successive RT phases. Thus, increases in responding that occurred during the RT phases were a product of a previous history rather than generalized increases in responding due to extinction.

Based on the results of Experiments 2a and 2b, if Control responding is observed during the RT phase of a three-phase resurgence procedure, then the source of such responding is most likely a previous history of reinforcement (see also, Bruzek et al. 2009). Therefore, it may be more appropriate to consider resurgence as hierarchical in that when a current response is extinguished, multiple historical responses may occur in a hierarchical or sequential manner, with the order determined by both their training order and the particular training conditions under which they were established. Investigating the determinants of the resurgence of multiple, sequentially established, responses, rather than seeking to identify the determinants of Control responding per se, places the latter in a more general framework and leads directly to the third experiment in this series.

\section{Experiment 3}

A hierarchical resurgence procedure, one in which multiple Target responses are 
reinforced sequentially, thus can be construed as a model for examining the conditions under which pre-experimental reinforcement histories may resurge. In this model, the initially reinforced response (i.e., Target-1 response) serves as a “Control” response and through manipulating the contingencies in effect during each phase, resurgence of more distally reinforced responses can be assessed. In Experiment 2a the initially reinforced response (i.e., Target 1) was maintained at a high rate, whereas the more recently reinforced response (i.e., Target 2) maintained no rate behavior. Although Target-1 responding resurged, this effect may have been enhanced by the atypical contingencies used to maintain Target 2. Thus, it is not clear whether Target-1 resurgence would be observed if the Target-2 response was maintained by a more typical reinforcement contingency (i.e., key pecks maintained by a VI schedule of reinforcement).

Therefore, the purpose of Experiment 3 was to further evaluate the role of the contingencies operating on Target-1 responding prior to the traditional three-phase resurgence procedure. This was done by using a two-component multiple schedule in which the Target-1 response in one component was maintained with a VR schedule and in the second component with a DRO schedule. Each component of the multiple schedule entailed a four-phase resurgence procedure which allowed for a direct comparison of the resurgence of a more distally (i.e., Target 1) and more recently reinforced response (i.e., Target 2). This experiment, therefore, served as a within-subject comparison of hierarchical resurgence as a function of Target 1response rates.

\section{Method}

\section{Subjects}

Three experimentally naive male White Carneau pigeons were maintained as described in 
Experiment 1.

\section{Apparatus}

Four operant chambers as described in the General Method section were used.

\section{Procedure}

Figure 10 shows a diagram of the procedure. It consisted of a two-component multiple schedule with each component comprising one of two daily sessions lasting until 60 reinforcers were obtained or 75-min elapsed. Each component was separated by a 3-hr intercomponent interval, during which the pigeons were returned to the vivarium. The two components of the multiple schedule were conducted in two different operant chambers, each of which was associated with a specific reinforcement history established during the Target-1-training phase described below. The component associated with the VR Target-1-training phase is referred to hereafter as the as the VR Component and the component associated with the DRO Target-1training phase as the DRO Component. The first session each day (i.e., DRO or VR component) was determined semirandomly with the constraint that the same order of components could not occur for four consecutive days. The sequence of the phases was as listed below. After the first RT phase, the complete four-phase sequence was repeated in both components.

Target 1-Training phase. Target-1 key pecks were reinforced according to a different schedule of reinforcement in each component. Responses to the Target and Alternative keys were recorded but were without programmed consequence. This phase was in effect for at least 13 sessions and until Target-1-response rates in both the VR and DRO components were deemed stable by visual inspection of the data during each session. Sessions terminated after 60 reinforcers or 75 min elapsed, whichever occurred first.

Target-1 VR training. During the VR component, always conducted in Chamber A, a VR 
30 was in effect for the VR Target-1 response (hereafter VRT1). The VR progression was generated as described in Experiment 2a.

Target-1 DRO training. During this component a variable DRO (VDRO) $x$-s schedule of reinforcement was in effect for the DRO Target-1 response (hereafter DROT1). To equate reinforcement rates during the DRO component, the VDRO intervals were generated using the interreinforcer intervals (IRIs) from the preceding VR component. If the pigeon pecked the DROT1 key, the DRO timer reset and reinforcement was postponed until the interval elapsed.

Target-2 Training phase. Target-2 responses were reinforced according to a VI 60-s schedule of reinforcement in both the VR and DRO components. Extinction was in effect for all other responses in each component. The Target-2 response in the DRO component was designated the DROT2 response and the Target-2 response in the VR component was designated the VRT2 response (hereafter VRT2). This condition was in effect until Target-1 response rates were less than $1 \mathrm{rpm}$ for three consecutive sessions and there was not a systematic downward trend in Target-2-response rates in both components.

AR phase. This phase was as described in the General Method section for both the VR and DRO component. The Alternative response in the DRO component was designated the ALTDRO response (hereafter ALTDRO) whereas the Alternative response in the VR component was designated the ALTVR response (hereafter ALTVR).

RT phase. This phase in both components was as described in the General Method section.

\section{Results}

Figure 11 shows response rates across phases for the VR component for each pigeon. The data are shown in this way to make the effects in each component clear. Table 4 shows the 
mean reinforcement rate for the last three sessions of each phase and component of the procedure as well as the number of sessions in each phase.

Figure 11 shows VRT1, VRT2, and ALTVR response rates. Responding reflected the schedule in effect during each phase. Thus, during the Target-1 training phase, response rates for VRT1 were high for each pigeon. More moderate response rates occurred for each pigeon during the Target-2 training and AR phases. Similar patterns of responding occurred during the replication of these phases as well. During the transition to the AR phase, VRT1 response rates transiently increased occurred with each pigeon. Figure 12 shows, on an expanded y-axis scale, VRT1 and VRT2 response rates during the last three sessions of the AR phase and all sessions of both RT phases. The VRT1 and VRT2 response rates increased within the first three to four sessions of both RT phases for Pigeons 15263 and 21055. For Pigeon 14475, however, VRT2 response rates increased only slightly relative to the last three sessions of the AR during the first four sessions of each RT phase. Pigeon 14475's VRT1 response rates increased later during each RT phase. Figure 13 shows the total number of VRT1 and VRT2 responses during the last three sessions of the AR phase and the first three sessions of the RT phase. During the first RT phase, the number of VRT2 responses was greater than the number of VRT1 responses for each pigeon. This was also true during the replication for Pigeons 14475 and 21055. For Pigeon 15263, however, the number of VRT1 responses exceeded the number of VRT2 responses. The patterns of resurgence for both VRT1 and VRT2 from the first session of each RT phase are shown in as cumulative records in Figure 14. During both the first session of both RT phases, VRT2 responding onset preceded VRT1 onset and there were more VRT2 responses than VRT1 responses.

To summarize, resurgence of both VRT1 and VRT2 occurred during each RT phase. 
During the initial RT phase, resurgence of VRT2 responding was greater than that of VRT1 responding. The onset of VRT2 resurgence occurred earlier during the initial RT session than did VTR1 resurgence. The resurgence pattern in the initial RT phase was replicated during the second resurgence test. The only exception was Pigeon 15263, where VRT1 responding exceeded that of VRT2. Thus, the more recently reinforced response, VRT2, generally resurged first and more vigorously than the initially reinforced, VRT1, response.

Figure 15 shows DROT1, DROT2, and ALTDRO response rates for each pigeon during the DRO component. With the VDRO contingency in effect during the Target-1 training phase, response rates were at or near zero during both iterations of the procedure. When VI 60-s schedule of reinforcement was in effect during the Target-2-training phase and AR phase, DROT2 and ALTDRO responding occurred at moderate rates. When extinction was implemented during the RT phase, DROT2 and DROT1 response rates increased for two to three sessions before dissipating. Figure 16 shows, on an expanded y-axis scale, DROT1 and DROT2 response rates for the last three sessions of the AR phase and the RT phase for both the initial RT phase and replication. For Pigeons 14475 and 21055 DROT1 response rates were higher than DROT2 response rates during the first session of the first RT phase, however, DROT1 response rates rapidly declined to zero by Session 2. As DROT1 response rates declined during the first RT phase, resurgence of DROT2 responding occurred for Pigeons 14475 and 21055. For Pigeon 15263 only DROT2 response rates increased during the initial RT phase. During the replication, resurgence of DROT2 responding occurred for each pigeon and there was no resurgence of DROT1. Figure 17 shows the total number of DROT1 and DROT2 responses during the last three sessions of the AR phase and the first three sessions of the RT phase. For all pigeons during the first and second RT phases, the number of DROT2 responses exceeded the number of 
DROT1 responses. The cumulative records of DROT1 and DROT2 responding during the first session of each RT phase for the DRO Component are shown in Figure 18. Although the number of DROT1 responses exceeded the number of DROT2 responses for Pigeons 14475 and 21055, the opposite was true for Pigeon 15263. For all three pigeons during the first session of the RT replication, the number of DROT2 responses exceeded DROT1 responses.

To summarize, resurgence of DROT2 occurred with each pigeon during both RT phases. Increases in DROT1-response rates, a response with a history of reinforcement of its absence, occurred for two of three pigeons during the first session of the first RT phase. For Pigeons 14475 and 21055, resurgence of DROT2 occurred during Sessions 2 and 3 of the first RT phase. During the replication of the RT phase, DROT2 responding resurged, but there was only minimal resurgence of DROT1 responding. As seen in Figure 18, DROT2 responding occurred earlier than DROT1 responding across both RT phases. For Pigeons 14475 and 21055, the number of DROT1 responses exceeded the number of DROT2 responses during the first session of the first RT phase and the opposite occurred during the replication. For all three pigeons, however, the total number of DROT2 responses during the first three sessions of both RT phases was greater than the number of DROT1 responses for each pigeon. Relative to VRT1 response rates, DROT1 response rates were lower during each RT phase for all three pigeons.

Figure 19 is a direct comparison of DROT1 and VRT1 response rates for the last three sessions of each AR phase and both RT phases. The data are shown in this way to compare resurgence of VRT1 and DROT1. In five of six cases, the resurgence of VRT1 exceeded that of DROT1 during each RT phase. For Pigeon 14475, however, DROT1 response rates were either similar or greater than VRT1-response rates during the first four sessions of each RT phase. Figure 20 shows the total number of VRT1 and DROT1 responses during the last three sessions 
of the AR phase and the first three sessions of the RT phase. For Pigeons 15263 and 21055, the number of VRT1 responses exceeded the number of DROT1 responses during the first and second RT phases. The number of DROT1 responses exceeded the number of VRT1 responses for Pigeon 14475 during the first RT phase but not for the replication of the RT phase.

\section{Discussion}

In Experiment 3 resurgence was compared within individual pigeons as a function of the Target-1-response rate and the sequential order in which the two Target responses were trained and maintained. There were two main results. First, the conditions under which the initially trained response was a determining factor of the magnitude and probability of its resurgence. That is, the resurgence of VRT1 generally exceeded increases in the number of DROT1 responses during the RT phase. Furthermore, the order in which the responses were trained also was critical in determining the probability and magnitude of resurgence. Second, for each pigeon, resurgence of the more recently reinforced response generally exceeded that of the initially trained response in both components. A similar effect occurred during the replication; however, increases in VRT1-response rates exceeded increases in VRT2-response rates during the replication of the RT phase for one pigeon. In the DRO Component, increases in DROT1response rates exceeded DROT2-response rate increases during the first session of the first RT phase for two of three pigeons. Given that the to-be resurged response for the DROT1 response was nonengagement, this increase may be interpreted as an instance of extinction-induced variability. During the replication, only increases in DROT2 response rates (i.e., resurgence) occurred during the RT phase. Taken together, these results show that more recently reinforced responses tend to resurge more substantially (i.e., a greater increase in response rates) than responses with a more distal reinforcement history. 
The results of Experiment 3 replicate and extend those of Experiment 2a. In both Experiments 2a and 3, when a more distally reinforced response was maintained at a high rate, then that high-rate response resurged. If such conditions are lacking, as occurred during the DRO Component, then increases in a response maintained under these conditions is unlikely. Unlike Experiment 2a, the more proximal reinforcement history of the Target-2 response in Experiment 3 involved key pecking as the reinforced response. Under the latter condition, Target 2-response rates were higher than those of Target 1, however, Target 1-response rate increases did occur. Unlike Experiment 2a, resurgence of the initially reinforced response occurred more reliably (i.e., resurgence of VRT1 occurred with all three pigeons) in Experiment 3.

The results of Experiment 3 replicate and extend those of Lattal et al. (Experiment 3, in press). In both the present experiment and Lattal et al. the reinforcement rate of the first-trained response was greater than that of the second trained response and in both experiments resurgence of the more recently trained response tended to be greater than that of the first trained response. Furthermore, for two of the three pigeons, resurgence of VRT1 occurred later during the RT phase, which is consistent with other experiments that have examined the resurgence of multiple responses (e.g., Lattal et al.). These results suggest that the order in which a response is trained is a potent variable in determining magnitude of resurgence.

The Experiment 3 results also address the question of the repeatability of the resurgence of multiple responses. Although repeatability of resurgence has been demonstrated previously (e.g., Cook \& Lattal, 2019; Kestner, Diaz-Salvat, St. Peter, \& Peterson, 2018; Lieving \& Lattal, 2003), little is known regarding the repeatability of the resurgence of serially-trained responses. In this experiment, the overall pattern of responding was similar across both resurgence test phases for two of three pigeons. That is, the resurgence of the second trained response tended to 
precede and exceed the resurgence of the initially reinforced response. For one pigeon, however, the initially reinforced response during the VR history session exceeded the resurgence of the second-reinforced response. Similar to Cook \& Lattal and Kestner et al., resurgence of VRT1 and VRT2 was relatively lower during the second RT phase as compared to the first.

A unique aspect of the four-phase resurgence procedure used in the current experiment, is that resurgence of the first-trained response occurs twice (see also Lattal et al., in press). This first instance of resurgence occurred during the transition between the Target 2 and AR phases. Response rates of the initially reinforced response increased for one session to two sessions in the VR component of the current experiment. Presumably, resurgence of the first-trained response occurred as a function of the more recently trained response being extinguished. This finding is notable because it provides further evidence resurgence can occur when there is concurrently available reinforcement within a session (e.g., Craig et al., 2017; Lieving \& Lattal, 2003; Oliver et al., 2018). That is, resurgence is not dependent upon extinction of alternative sources of reinforcement (see also Lattal et al., 2017).

Resurgence of more recently reinforced responses tends to occur more reliably than responses with more distal reinforcement histories, although the limits to this conclusion are unknown (cf. Bruzek et al., 2009). This recency effect may account for why the Target response tends to resurge more robustly than a Control response, even if an organism has some unspecified pre-experimental reinforcement history. It is important, however, to consider these unspecified reinforcement histories when interpreting Control-operandum responding. In the present experiment, the attempt was to experimentally establish a “pre-experimental” history to examine how a putative control response might be affected during resurgence testing of more recently reinforced responding. The conditions under which the Control response was 
maintained prior to its elimination, as based on the results of the current experiment, is affected the probability and magnitude of its resurgence.

\section{General Discussion}

The Control response is considered the gold-standard method for isolating resurgence from other forms of extinction-induced variability in operant-resurgence procedures. The way in which it is used to do so is by comparing the relative increases in the Control and Target responses during the RT phase. If Target responding exceeds Control responding, then the former is interpreted as a product of the reinforcement history for that response in the resurgence procedure. Conversely, if Control responding exceeds Target responding, this is interpreted as an instance of extinction-induced variability. An alternative account of Control responding is that it is an outcome of behavioral history (cf. Bruzek et al., 2009).

Therefore, the present experiments examined the effects of different behavioral histories on resurgence, with a specific focus on the provenance of Control responding, when it occurs during the RT phase of a resurgence procedure. In Experiment 1, Control responding was assessed as a function of the Training phase of a three-phase resurgence procedure. When the Target response was maintained by a DRO schedule, as what was done in Experiment 1, Control responding occurred for two of four pigeons during the Training phase. Although the results of Experiment 1 may be interpreted as an instance of resurgence in that the target response expected to resurge was "not responding," the increases in Control responding observed with two pigeons can be interpreted in two ways. First, it is possible that the increases in Control responding were instances of induction. Alternatively, Control responding in Experiment 1 may have been the resurgence of a response with a pre-experimental reinforcement history as each pigeon had a history of keypecking on various schedules of reinforcement. 
A four-phase resurgence procedure was used to model behavioral histories that may produce Control responding in Experiments 2a, 2b, and 3. In these experiments different Target responses were maintained under a variety of conditions to identify how these contingencies affected the probability and magnitude of resurgence during the RT phase. In Experiments 2a and $2 \mathrm{~b}$ specific reinforcement histories were sequentially established and this led to their differential return during a resurgence test. Considering these differential prior histories of reinforcement as a model for the histories that might be associated with the Control response, the results reveal how control responding itself might reflect such remote reinforcement histories. Finally, in Experiment 3, resurgence within individual pigeons was compared as a function of the Target-1 response rate and the sequential order in which the two Target responses were maintained. Resurgence of a response with a more recent reinforcement history tended to resurge more robustly than responses with a temporally distant reinforcement history. If the first-trained response is analogous to a Control response, the Experiment 3 results shed light on the presence and absence of control responding during a resurgence test. First, the absence of control responding could reflect an absence of induction, or it could reflect the failure of a distally trained response to resurge. Second, the presence of Control-operandum responding could reflect either resurgence of a previously reinforced response or a general increase of responding brought on by the extinction of Alternative responding.

One methodological feature of the current experiments that warrants further discussion is the resurgence of a response maintained by a DRO contingency. In all four experiments, a DRO contingency was used in either during the Target (Experiment 1), Target-2 (Experiment 2a and 2b), or Target-1 (Experiment 3) training phase. In each of these cases, the reinforced operant was non-engagement and, as a result, it was not expected that increases in responding for that 
response would be observed during the RT phase of each experiment. In cases in which responding did occur on the response in which the DRO contingency was in effect, then such instances of responding may be interpreted as extinction-induced variability. The absence of responding, however, cannot be definitively described as an instance of resurgence of nonresponding. This is because zero rates of behavior for the response with a DRO history of reinforcement occurred in each experiment. Thus, it is not possible to quantify the change in behavior between the AR phase and the RT, which is necessary to determine a resurgence effect. It is possible, however, that if a video camera was used to record "other” behavior that was maintained by the DRO contingency when that contingency was in effect in each of the current experiments it would then be possible to ascertain whether "other" behavior did resurge during the RT phase.

In the sections that follow, the results are discussed in relation to a conventional interpretation of Control responding and the recurrence of multiple, sequentially trained, responses. The results also are discussed in relation to Control responses in human and nonhuman response experiments. Finally, the discussion concludes with a brief consideration of the results in the context of their potential application.

\section{Interpretation of Control Responding}

As stated in the preceding section, conventional interpretations of Control responding focus on the presence or absence of Control responding as either reflecting the lack of a reinforcement history of that response or as an instance of extinction-induced variability. These interpretations, however, fail to account for how or why Control responding does or does not occur during the RT phase. This is because there are multiple possibilities that can account for the presence or absence of Control responding, some of which will be discussed in the following 
section.

The absence of Control responding during the RT phase does not necessarily mean that its absence is due to the lack of a reinforcement history for the Control response. Such absence could reflect nothing more than the Control response being an indistinguishable, behaviorally meaningless, part of the environment, no different from any other feature of the operant chamber such as a wall or a houselight that fails to capture responding. Alternatively, extinction of the Alternative response during the RT phase may evoke the occurrence of other behavior that competes with Control responding. The behavior evoked by extinction may be such that it is not readily measured by the Control response and as a result Control responding would not be observed. This latter observation, however, is speculative, as the current investigation was not designed to measure the emergence of topographically dissimilar responding induced by extinction. If, however, the operant chambers were equipped with cameras then topographical changes in behavior during the RT phase could be measured.

If Control responding occurs during the RT phase, then it could be an instance of response generalization or induction (cf. Catania, 2013, p. 118; Skinner, 1953, p. 93). Although these terms are used interchangeably by both Catania and Skinner, in the context of the current discussion response generalization and induction could be taken to refer to two distinct phenomena. Response generalization can refer to the occurrence of the same operant response (e.g., key pecking or lever pressing) in a context different from the original training condition. It also can refer to a variant of the trained response occurring to the same operandum on which the original response was trained. An example of the latter is variations in response force around the originally trained force (Notterman \& Mintz, 1965). Induction, however, can refer to a more general increase in behavior as a function of worsening conditions of reinforcement. An 
example of induction is an increase in response variability as function of extinction (e.g., Antonitis, 1951; Ekerman \& Lanson, 1969). In the case of responding on a control operandum in an operant-resurgence procedure, it may be more apt to characterize such responding as an instance of response generalization rather than induction. This is because the Control response is typically structurally similar to the operandum reinforced (e.g., the Target or Alternative responses) within the resurgence procedure.

Concerning the interpretation of Control responding, the results of the current experiments show how such responding might develop as a function of a distal reinforcement history. The extent to which Control responding occurs, if there is a distal reinforcement history for that response, however, is a function not only of that pre-experimental history but also of the proximal reinforcement conditions under which responding is maintained. In Experiment 1, Control-operandum responding occurred with two of four pigeons, which suggests that preexperimental histories may manifest during the RT phase and in other cases they may not. Alternatively, for the two pigeons in which Control responding did not occur, the reinforcement history for the Control response may have been so temporally remote to the current experiment that this history became moot in relation to the Control response in current procedure. Although each of the pigeons in Experiment 1 had a history of keypecking on various schedule of reinforcement, it was unknown as to whether the pigeons had experience pecking the designated Control key. Thus, there is still the possibility that Control responding observed in Experiment 1 was an instance of response generalization. In Experiments 2a and 2b, an explicit reinforcement history of a response for experimentally naïve pigeons and rats resulted in its resurgence. Furthermore, the magnitude of that resurgence exceeded that of a second response that lacked a reinforcement history (i.e., a true Control response). Furthermore, responding did not generalize 
to a response in which the proximal reinforcement history of that response was nonengagement. Thus, Experiments 1, 2a, and 2b highlight the importance of both proximal and distal reinforcement histories on Control responding during the RT phase.

One of the major implications of the results of Experiment 2a and $2 b$ is that when Control responding occurs and such responding is substantial, as in the case of human-operant resurgence experiments (e.g., Bolivar et al., 2017; Sweeney \& Shahan, 2016), then the likely source of such responding is a distal reinforcement history. Although speculative, based on the results obtained in Experiments 2a and 2b, the Control responding that occurred in Experiment 1 could have been the manifestation of a distal reinforcement history and not simply response generalization. Furthermore, the emergence of responses with more distal reinforcement histories in Experiments 1, 2a, and 2b may be a function of the more proximal reinforcement history of nonengagement for the second trained response. As found in Experiment 3, Control responding may be obscured by a more recent reinforcement history even when the more distally reinforced behavior is maintained at a high rate prior to its elimination.

Although Control responding typically is interpreted as either an instance of extinctioninduced variability when it occurs or a reflection of the absence of a reinforcement history when it does not occur, the results of the current experiments, taken together, highlight some issues regarding these interpretations. Specifically, in instances in which Control responding occurs during the RT phase it is neither sufficient nor accurate to conclude that such responding is simply an instance of extinction-induced variability (i.e., induction as defined above) as it may be more accurate to describe such responding as response generalization (as defined above). Furthermore, it may be possible that in cases of substantial Control responding during the RT phase that the source of such responding is a distal reinforcement history. If this is the case, then 
the interaction between these distal reinforcement histories and more proximal contingencies may determine the magnitude of Control responding during the RT phase.

\section{Hierarchical Resurgence}

Control-operandum responding in a resurgence test can be considered as an example of hierarchical resurgence, which is defined as the recurrence of serially trained target responses as a function of the termination or worsening of alternative reinforcement. Through interpreting Control responding as an instance of hierarchical resurgence, then the conditions that affect the probability and magnitude of the Control response can be elucidated in a systematic manner. Thus, by this interpretation, Control responding during the RT phase is a function of the conditions in effect when the response was maintained as well as the sequential order in which the response was trained. In the discussion that follows, Control responding is interpreted in light of the current results as well as in the context of hierarchical resurgence.

The outcome of a hierarchical procedure typically is interpreted in two ways. If, during an RT phase a more recently-reinforced response resurged first, then such an effect is termed a recency effect. If, however, the first-trained (or earlier trained) response resurged more or first, this is described as a primacy effect (e.g., Bruzek et al., 2009; Reed \& Morgan, 2006). In the current experiments, variables that might help account for recency and primacy effects, as a model of Control responding, were investigated using two approaches. In Experiment 1, Control responding as a function of the proximal reinforcement contingencies in the resurgence procedure were investigated using experimentally experienced animals. This was designed to ascertain whether a pre-experimental history may manifest as Control responding using a threephase resurgence procedure. With Experiments 2a, 2b, and 3, however, a four-phase resurgence procedure was used to model Control responding. In these experiments, an explicit 
reinforcement history was established for a first-trained response to serve as a model of the Control response (i.e., the Target 1 response in each of these experiments). Then, in Experiments 2a, 2b, and 3, the resurgence of this first-trained response was assessed in relation to the conditions under which it was maintained as well as in relation to the more proximal reinforcement conditions for a second-trained response. Thus, the interaction of proximal and distal reinforcement histories and their subsequent effects on the resurgence of Control responding could be assessed.

The results of the different experiments can be interpreted as either a primacy or recency effect. The data from Experiment 1 provide examples of both. For two pigeons, Control responding (i.e., the response which lacked an intra-experimental reinforcement history) occurred and, given that no intra-experimental reinforcement history was established for the Control response, the occurrence of Control-operandum responding may be interpreted as a primacy effect. That is, Control responding may have been a manifestation of a preexperimental reinforcement history rather than response generalization. Conversely, for the two pigeons in which Control responding did not occur, such an effect could be a recency effect. The recency interpretation of these results is warranted as it may have been due to the absence of a reinforcement contingency for the Control response and the DRO Contingency in effect for the Target response. In Experiments 2a and 2b, however, the results suggest a primacy effect. That is, the first-trained response resurged more than the second-trained response. It should be noted, however, that this primacy effect may have been a function of the DRO contingency in effect for the second-trained response in this procedure. Finally, in Experiment 3 there is a preponderance of evidence suggesting a recency effect. That is, resurgence of a more recently reinforced response preceded and exceeded the resurgence of a response with a more distal reinforcement 
history. The results of Experiment 3 replicate those reported by Lattal et al. (in press).

The present results highlight the role of proximal and distal reinforcement histories in the interpretation of Control responding during the RT phase. In Experiments 2a and 2b, a primacy effect may have been a function of the more recent reinforcement history of nonengagement (i.e., DRO contingency) of the Target response, whereas the recency effect obtained in Experiment 3 may have been due to the key-peck contingency (i.e., VI schedule) for the more recently reinforced response. Thus, in a case in which nonengagement is the second-trained response, it is likely that Control responding may occur under those conditions. Conversely, if the secondtrained response is topographically similar to the first-trained response, then it may be the case that Control responding may not occur under these conditions. Therefore, Control responding during the RT phase is a function of the distal reinforcement history of that response as well the contingencies in effect for the more recently reinforced response.

The recency effect reported in Experiment 3 as well as Lattal et al. (in press) is supported by the Resurgence as Choice (RaC) model of resurgence (Shahan \& Craig, 2017). The RaC model of resurgence is a quantitative account of resurgence based on the matching law (Herrnstein, 1970), in which resurgence is conceptualized as resulting from the dynamic relation between the relative value of the Alternative and Target responses during the RT phase. Value, in the $\mathrm{RaC}$ model, is a product of the reinforcement rate and the relative recency of reinforcement, as based on the temporal weighting rule, of each response. The latter rule asserts that events are weighted based on their relative recency. Thus, more recent events are weighed more than more distal events (see Mazur, 1996 for a review). Therefore, according to the RaC model, the relative difference in time between when the first-trained response was last reinforced and when a more recently trained response was last reinforced may account for the differential 
resurgence of these two responses during the RT phase of a hierarchical-resurgence procedure. Although the present experiments were not designed to test the $\mathrm{RaC}$ model, the results are consistent with the temporal weighting rule and the RaC model more broadly.

The methods of each of the present experiments have been described as modeling the relation between particular reinforcement histories and the likelihood of Control responding. The results indicate that Control responding is a function of the contingencies in effect when the Control response was established and maintained prior to its use or measurement as a control response, the sequential order in which the Control and Target responses are trained, and the conditions under which the Target response was maintained. The critical factor, however, is the sequential order in which the Control and Target responses are trained. As suggested by the $\mathrm{RaC}$

model, more recently reinforced events are weighed more heavily, thus increasing the probability of their occurrence during the RT phase. This recency effect as predicted by the RaC model, may account for the absence of Control responding in nonhuman-operant resurgence procedures. Other variables, however, such as the length of training (e.g., Bruzek et al., 2009) or the way in which the more recently reinforced response is maintained (e.g., Experiments 2a and 2b of the current investigation) may result in a primacy effect. Thus, under these conditions it is likely that Control responding may be observed.

\section{Levels of Control Responding: Differences in Human and Nonhuman Resurgence Experiments}

As noted in the Literature Review when a Control response is included in a humanoperant resurgence procedure, Control responding is generally equal to or exceeds Target responding during the RT phase (e.g., Bolivar et al., 2017; Sweeney \& Shahan, 2016). The occurrence of substantial Control responding during these procedures makes it difficult to parse 
out the resurgence of an intra-experimental reinforcement history from extinction-induced variability. Although it is likely that the response generalization principle may account for minimal Control responding in nonhuman resurgence experiments, as was the case, for example, in Experiments 2a and 2b, it is likely that in human-operant resurgence experiments the rather extensive Control responding is a product of a pre-experimental reinforcement history. Thus, Control responding in the case of human-operant resurgence experiments may be better described as the emergence of functionally equivalent, but topographically distinct responses that are evoked by the onset of extinction (see also, Ho, Bai, Keevy, \& Podlesnik, 2018). The assertion that Ho et al. make regarding the interpretation of Control responding is supported, in part, by the arrangements typically used in human-operant resurgence procedures. As described in the introduction, the experimental arrangements used in human-operant response procedures are described as "novel"; however, these experiments make use of responses such as button presses or clicks to a computer screen. The populations that commonly participate in humanoperant resurgence experiments (i.e., undergraduate students) engage with these types of responses on a daily basis.

The extensive reinforcement history people have with the types of operanda used in human-operant resurgence procedures thus poses a problem of interpreting resurgence in terms of the reinforcement history established in the resurgence procedure. As discussed in the introduction, the length of training affects the probability and magnitude of resurgence (e.g., Bruzek et al., 2009; Reed \& Morgan, 2006; Doughty, Cash, Finch, Holloway, \& Wallington, 2010). Given the relative brevity of the reinforcement history established for Target responding in a typical human-operant resurgence experiment, it is not surprising that Control responding often exceeds Target responding in these experiments. In light of the experiments in this 
dissertation, however, there is a possibility that although a primacy effect seems to occur routinely in human-operant resurgence experiments, the onset of the Target response during the RT phase may precede the onset of Control responding in such experiments. This possibility has not been examined in these experiments.

\section{Potential Areas of Application}

In the applied literature, the experimental analysis of resurgence has focused on identifying conditions under which targeted-problem behavior recurs when there are treatmentintegrity failures (e.g., Marsteller \& St. Peter, 2014; Volker et al., 2009) and in the identification of strategies to reduce the probability of resurgence of targeted-problem behavior (e.g., Fuhrman, Fisher, \& Greer, 2016). Although the focus of this research has been on the resurgence of targeted-problem behavior, increases in non-targeted aberrant behavior may occur during treatment integrity failures or extinction. For example, Ho et al. (2018) reported increases in nontargeted problem behavior during two different resurgence test conditions. Ho et al. used a three-phase procedure in which the resurgence of the Target response (i.e., placing a ball in a box) was assessed during extinction and a progressive-ratio (PR) schedule during the RT phase. During both the PR and extinction RT phases, resurgence of the Target response occurred, however, increases in emotional and other responses (i.e., responses that were functionally equivalent to the Target or Alternative responses such as requests for the reinforcer) relative to the Training and AR phases occurred. Similarly, Sullivan et al. (in press) reported increases in untargeted behavior during the onset of extinction. Using a three-phase procedure, Sullivan et al. reported that not only did Target-problem behavior resurge, but so did corresponding increases in nontargeted problem behavior when the Alternative response was no longer reinforced. Thus, the resurgence of responses with distal reinforcement histories do resurge in both translational and 
clinical settings. Furthermore, the results reported by Ho et al. and Sullivan et al. are similar to those reported in the present experiments; resurgence is hierarchical in that last-trained responses tend to recur before earlier trained responses.

In applications of resurgence research, it may be that a hierarchy of responses resurge when extinction is implemented. Similar to the present findings, more recently reinforced responses (i.e., targeted-problem behavior) may resurge first followed by the resurgence of responses with a more distal reinforcement history (see also, Ho et al., 2018). If this is the case, this may help to understand increases in response variability observed during the application of extinction in intervention settings, such as the case with the extinction burst or extinctioninduced aggression (e.g., Lerman \& Iwata, 1995), which can be used to inform more effective interventions. In the case of substance abuse, when an individual encounters a treatment challenge such as a missed dose of suboxone or a delayed voucher delivery, as in the case of a contingency management intervention, then an individual engage in previously reinforced behavior based on its relative reinforcement history.

Similarly, the resurgence of response hierarchies may be a useful approach to analyzing complex verbal behavior such as problem solving (e.g., Epstein, 1985). For example, if a certain approach to solving a problem is no longer successful, an individual may start to use strategies that had previously been successful. In light of the current experiments, it may be the case that the problem-solving strategies used by an individual may recur in a hierarchical manner. That is, more recently successful strategies may be attempted followed by strategies with a more distal reinforcement history.

\section{Conclusion}

The resurgence of a previously reinforced response is a function of both the 
reinforcement history of the organism and prevailing environmental contingencies. Such resurgence is evaluated based on the relative difference between a response with an experimentally established, relatively recent, reinforcement history and a Control response that lacks an intra-experimental reinforcement history. If Control responding exceeds that of the Target response, then the Target responding is interpreted as an instance of extinction-induced variability (Epstein, 1983; Sweeney \& Shahan 2016). The results of the present experimental analysis of control responding, however, suggest that when such responding occurs it as an instance of resurgence of a response with a more distal reinforcement history. Thus, the present results, taken together, call into question the utility of the Control response as true control in an operant-resurgence procedure.

It remains an open question as to whether the Control response should continue to be included in operant-resurgence experiments. The Control response may have utility in isolating resurgence from response generalization or induction, however, in light of the current results, this may be limited to nonhuman-animal resurgence experiments. As in these types of experiments, Control responding is minimal due to the strictly controlled reinforcement histories of the animals (e.g., Craig et al., 2017; Oliver et al., 2018; Sweeny \& Shahan, 2013). In the case of human-operant resurgence research, however, the Control response may just add unnecessary complexity to the interpretation of the outcome of such procedures. Simply because Control responding occurs does not necessarily suggest that such responding is indicative of extinctioninduced variability as is commonly interpreted (e.g., Sweeny \& Shahan, 2016), as such response may occur due to a variety of factors. Therefore, the question as to whether a Control response should be included in an operant-resurgence procedure should be made in relation to the research question that is being addressed, rather than the default methodological feature of the 
experiment. 


\section{References}

Alessandri, J., Lattal, K. A., \& Cançado, C. X. (2015). The recurrence of negatively reinforced responding of humans. Journal of the Experimental Analysis of Behavior, 104(3), 211222. doi:10.1002/jeab.178

Antonitis, J. J. (1951). Response variability in the white rat during conditioning, extinction, and reconditioning. Journal of Experimental Psychology, 42, 273-281. doi:10.1307/h0060407

Brown, P. L., \& Jenkins, H. M. (1968). Auto-shaping of the pigeon's key-peck. Journal of the Experimental Analysis of Behavior, 11, 1-8. doi:10.1901/jeab.1968.11-1

Bolivar, H. A., Cox, D. J., Barlow, M. A., \& Dallery, J. (2017). Evaluating resurgence procedures in a human operant laboratory. Behavioural Processes, 140, 150-160. doi:10.1016/j.beproc.2017.05.004

Bruzek, J. L., Thompson, R. H., \& Peters, L. (2009). Resurgence of infant caregiving responses. Journal of the Experimental Analysis of Behavior, 92(3), 327-343. doi:10.1901/jeab.2009-92-327

Catania, A. C. (2013). Learning. Cornwall-on-Hudson, NY: Sloan Publishing LLC.

Cleland, B. S., Foster, T. M., \& Temple, W. (2000). Resurgence: The role of extinction. Behavioural Processes, 52, 117-129. doi:10.1016/s0376-6357(00)00131-5

Cook, J. E., \& Lattal, K. A. (2019). Repeated, within-session resurgence. Journal of the Experimental Analysis of Behavior, 111, 28-47. doi:10.1002/jeab.496 
Craig, A. R., Browning, K. O., Nall, R. W., Marshall, C. M., \& Shahan, T. A. (2017).

Resurgence and alternative-reinforcer magnitude. Journal of the Experimental Analysis of Behavior, 107(2), 218-233. doi:10.1002/jeab.245

Doughty, A. H., Cash, J. D., Finch, E. A., Holloway, C., \& Wallington, L. K. (2010). Effects of training history on resurgence in humans. Behavioural Processes, 83(3), 340-343. doi:10.1016/j.beproc.2009.12.001

Doughty, A. H., da Silva, S. P., \& Lattal, K. A. (2007). Differential resurgence and response elimination. Behavioural Processes, 75, 115-128. doi:10.1016/j.beproc.2007.02.025

Eckerman, D. A., \& Lanson, R. N. (1969). Variability of response location for pigeons responding under continuous reinforcement, intermittent reinforcement. Journal of the Experimental Analysis of Behavior, 12, 73-80. doi:10.1901/jeab.1969.12-73

Epstein, R. (1983). Resurgence of previously reinforced behavior during extinction. Behaviour Analysis Letters, 3, 391-397.

Epstein, R. (1985). Extinction-induced resurgence: Preliminary investigations and possible applications. The Psychological Record, 35, 143-153. doi:10.1007/BF03394918

Flesher, M., \& Hoffman, H. S. (1962). A progression for generating variable-interval schedules. Journal of the Experimental Analysis of Behavior, 5, 529-530. doi:10.1901/jeab.1962.5-529

Fuhrman, A. M., Fisher, W.W., \& Greer, B. D. (2016). A preliminary investigation on improving functional communication training by mitigating resurgence of destructive behavior. Journal of Applied Behavior Analysis, 49, 884-899. doi:10.1002/jaba.338 
Herrnstein, R. J. (1970). On the law of effect. Journal of the Experimental Analysis of Behavior, 13, 243-266. doi:10.1901/jeab.1970.13-243

Ho, T., Bai, J. Y. H., Keevy, M., \& Podlesnik, C. A. (2018). Resurgence when challenging alternative behavior with progressive rations in children and pigeons. Journal of the Experimental Analysis of Behavior, 110, 474-499. doi:10.1002/jeab.474

Jarmolowicz, D. P., \& Lattal, K. A. (2014). Resurgence under delayed reinforcement. The Psychological Record, 64(2), 189-193. doi:10.1007/s40732-014-0040-0

Kestner, K. M., Diaz-Salvat, C. C., St Peter, C. C., \& Peterson, S. M. (2018). Assessing the repeatability of resurgence in humans: Implications for the use of within-subject designs. Journal of the Experimental Analysis of Behavior, 110, 545-552. doi:10.1002/jeab.477

Kestner, K. M., Redner, R., Watkins, E., \& Poling, A. (2015). The effects of punishment on resurgence in laboratory rats. The Psychological Record, 65, 315-321. doi:10.1007/s40732-014-0107-y

Kincaid, S. L., Lattal, K. A., \& Spence, J. (2015). Super-resurgence: ABA renewal increases resurgence. Behavioural Processes, 115, 70-73. doi:10.1016/j.beproc.2015.02.013

Lambert, J. M., Bloom, S. E., Samaha, A. L. \& Dayton, E. (2017). Serial functional communication training: Extending serial DRA to mands and problem behavior. Behavioral Interventions, 32, 311-325. doi:10.1002/bin.1493

Lattal, K. A., Cançado, C. X., Cook, J. E., Kincaid, S. L., Nighbor, T. D., \& Oliver, A. C. (2017). On defining resurgence. Behavioural Processes, 141, 85-91. doi:10.1016/j.beproc.2017.04.018 
Lattal, K. A., Solley, E. A., \& Cançado, C. X., \& Oliver (in press). Hierarchical Resurgence. Journal of the Experimental Analysis of Behavior.

Lattal, K. A., \& St. Peter Pipkin, C. (2009). Resurgence of previously reinforced responding: Research and application. Behavior Analysis Today, 10, Retrieved from http://www.behavior-analyst-today.net

Lerman, D. C., \& Iwata, B. A. (1995). Prevalence of the extinction burst and its attenuation during treatment. Journal of Applied Behavior Analysis, 28, 93-94. doi:10.1901/jaba.1995.28-93

Lieving, G. A., \& Lattal, K. A. (2003). Recency, repeatability, and reinforcer retrenchment: An experimental analysis of resurgence. Journal of the Experimental Analysis of Behavior, 80(2), 217-233. doi:10.1901/jeab.2003.80-217

Martsteller, T. M., \& St. Peter, C. C. (2014). Effects of fixed-time reinforcement schedules on resurgence of problem behavior. Journal of Applied Behavior Analysis, 47, 455-469. doi:10.1002/jaba.134

Mazur, J. E. (1996). Past experience, recency, and spontaneous recovery in choice behavior. Animal Learning \& Behavior, 24, 1-10. doi:10.3758/BF03198948

Mulick, J. A., Leitenberg, H., \& Rawson, R. A. (1976). Alternative response training, differential reinforcement of other behavior, and extinction in squirrel monkeys (Saimiri sciureus). Journal of the Experimental Analysis of Behavior, 25, 311-320. doi:10.1901/jeab.1976.25-311

Notterman, J. M., \& Mintz, D. E. (1965). Dynamics of response. New York, NY: Wiley. 
Okouchi, H. (2015). Resurgence of two-response sequences punished by point-loss response cost in humans. Revista Mexicana de Análisis de la Conducta, 41, 137-154. doi: 10.5514/rmac.v41.i2.63744

Oliver, A. C., Nighbor, T. D., \& Lattal, K. A. (2018). Reinforcer magnitude and resurgence. Journal of the Experimental Analysis of Behavior, 110, 440-450. doi:10.1002/jeab.481

Podlesnik, C. A., Jimenez-Gomez, C., \& Shahan, T. A. (2006). Resurgence of alcohol seeking produced by discontinuing non-drug reinforcement as an animal model of relapse. Behavioural Pharmacology, 17(4), 369-374. doi:10.1097/01.fbp.0000224385.09486.ba

Reed, P., \& Morgan, T. A. (2006). Resurgence of response sequences during extinction in rats shows a primacy effect. Journal of the Experimental Analysis of Behavior, 86, 307-315. doi:10.1901/jeab.2006.20-05

Shahan, T. A., \& Craig, A. R. (2017). Resurgence as choice. Behavioural Processes, 141, 100127. doi:10.1016/j.beproc.2016.10.006

Shahan, T. A., \& Lattal, K. A. (1998). On the functions of the changeover delay. Journal of the Experimental Analysis of Behavior, 69, 141-160. doi:10.1901/jeab.1998.69-141

Skinner, B. F. (1953). Science and Human Behavior. New York, NY: The Free Press.

da Silva, S. P., Maxwell, M. E., \& Lattal, K. A. (2008). Concurrent resurgence and behavioral history. Journal of the Experimental Analysis of Behavior, 90, 313-331. doi:10.1901/jeab.2008.90-313 
Sullivan, W., Saini, V., DeRosa, N., Craig, A., Ringdahl, J., \& Roane, H. (in press). Measurement of nontargeted problem behavior during investigations of resurgence. Journal of Applied Behavior Analysis.

Sweeney, M. M., \& Shahan, T. A. (2013). Effects of high, low, and thinning rates of alternative reinforcement on response elimination and resurgence. Journal of the Experimental Analysis of Behavior, 100, 102-116. doi:10.1002/jeab.26

Sweeney, M. M., \& Shahan, T. A. (2016). Resurgence of target responding does not exceed increases is inactive responding in a forced-choice alternative reinforcement procedure in humans. Behavioural Processes, 124, 80-92. doi:10.1016/j.beproc.2015.12.007

Volkert, V. M., Lerman, D. C., Call, N. A., \& Trosclair-Lasserre, N. (2009). An evaluation of resurgence during treatment with functional communication training. Journal of Applied Behavior Analysis, 42(1), 145-160. doi:10.1901/jaba.2009.42-145

Winterbauer, N. E., \& Bouton, M. E. (2010). Mechanisms of resurgence of an extinguished instrumental behavior. Journal of Experimental Psychology: Animal Behavior Processes, 36(3), 343-353. doi:10.1037/a0017365 
Table 1.

Response Key Designations for Experiment 1

\begin{tabular}{cccc} 
& \multicolumn{3}{c}{ Response-Key Designation } \\
\cline { 2 - 4 } Pigeon & Alternative & Target & Control \\
\hline 267 & Left & Center & Right \\
567 & Right & Left & Center \\
847 & Center & Right & Left \\
9553 & Center & Right & Left
\end{tabular}

Note: Each key was with a specific key-light color that varied depending on the operant chamber in which the experiment will be conducted. For Pigeons 267 and 567 the key-light colors were as follows: White (left), Center (green), Right (red). For Pigeons 847 and 9553 the key-light colors were as follows: Red (left), Center (blue), Right (green). 
Table 2.

Response-Key Designations for Experiment 2

\begin{tabular}{ccccc} 
& & \multicolumn{3}{c}{ Response-Key Designation } \\
\cline { 3 - 4 } Pigeon & Control & Target 1 & Target 2 & Alternative \\
\hline 18057 & Right & Left & Center & Back \\
18263 & Left & Right & Center & Back \\
35424 & Right & Left & Center & Back
\end{tabular}

Note: The key lights were transilluminated with the following colors: blue-back, center-white, left-purple, and right -orange. 
Table 3.

Response Key Designations for Experiment 3

Response Key Designation

\begin{tabular}{|c|c|c|c|c|c|c|}
\hline \multirow[b]{2}{*}{ Pigeon } & \multicolumn{3}{|c|}{ VR Component } & \multicolumn{3}{|c|}{ DRO Component } \\
\hline & Target 1 & Target 2 & Alternative & Target 1 & Target 2 & Alternative \\
\hline 14475 & Right & Left & Center & Left & Right & Center \\
\hline 15263 & Left & Right & Center & Left & Right & Center \\
\hline 21055 & Left & Right & Center & Right & Left & Center \\
\hline
\end{tabular}

Note: All key designations and key-light colors remained the same across all phases of the experiment for each pigeon. Each session was separated by a 4-hr intersession interval. 
Table 4.

Reinforcement Rates for Each Component of Experiment 3

\begin{tabular}{|c|c|c|c|c|}
\hline Pigeon & Phase & Sessions & $\begin{array}{c}\text { Mean VR Component } \\
\text { Reinforcement Rate } \\
\text { (active key) }\end{array}$ & $\begin{array}{c}\text { Mean DRO Component } \\
\text { Reinforcement Rate } \\
\text { (active key) }\end{array}$ \\
\hline \multirow[t]{4}{*}{14475} & Target $1 \mathrm{~T}$ & 13 & $2.96(0.24)$ & $3.11(0.03)$ \\
\hline & Target $2 \mathrm{~T}$ & 13 & $0.99(0.04)$ & $0.98(0.05)$ \\
\hline & Alt SR & 18 & $1.00(0.06)$ & $0.96(0.02)$ \\
\hline & $\mathrm{RT}$ & 10 & - & - \\
\hline \multirow[t]{4}{*}{15263} & Target $1 \mathrm{~T}$ & 13 & $6.07(0.54)$ & $5.91(0.42)$ \\
\hline & Target $2 \mathrm{~T}$ & 13 & $1.00(0.03)$ & $0.98(0.01)$ \\
\hline & Alt SR & 16 & $0.97(0.03)$ & $0.96(0.02$ \\
\hline & $\mathrm{RT}$ & 10 & - & - \\
\hline \multirow[t]{4}{*}{21055} & Target $1 \mathrm{~T}$ & 13 & $3.05(0.16)$ & $3.01(0.01)$ \\
\hline & Target $2 \mathrm{~T}$ & 13 & $0.98(0.03)$ & $0.97(0.04)$ \\
\hline & Alt SR & 24 & $0.99(0.03)$ & $0.99(0.02)$ \\
\hline & $\mathrm{RT}$ & 10 & - & - \\
\hline \multicolumn{5}{|l|}{ Replication } \\
\hline \multirow[t]{4}{*}{14475} & Target $1 \mathrm{~T}$ & 13 & $2.36(0.48)$ & $2.09(0.05)$ \\
\hline & Target $2 \mathrm{~T}$ & 15 & $0.97(0.04)$ & $0.99(0.03)$ \\
\hline & Alt SR & 24 & $0.98(0.04)$ & $0.98(0.01)$ \\
\hline & $\mathrm{RT}$ & 14 & - & - \\
\hline \multirow[t]{4}{*}{15263} & Target $1 \mathrm{~T}$ & 14 & $4.37(0.62)$ & $4.29(0.67)$ \\
\hline & Target $2 \mathrm{~T}$ & 14 & $0.98(0.03)$ & $0.98(0.02)$ \\
\hline & Alt SR & 19 & $0.97(0.03)$ & $0.97(0.03)$ \\
\hline & $\mathrm{RT}$ & 10 & - & - \\
\hline \multirow[t]{4}{*}{21055} & Target $1 \mathrm{~T}$ & 14 & $4.22(0.20)$ & $3.85(0.13)$ \\
\hline & Target $2 \mathrm{~T}$ & 16 & $0.98(0.03)$ & $0.98(0.05)$ \\
\hline & Alt SR & 18 & $0.99(0.05)$ & $0.99(0.04)$ \\
\hline & $\mathrm{RT}$ & 11 & - & - \\
\hline
\end{tabular}

Note: Average reinforcement rates (reinforcers per minute) were calculated using the last three sessions of each phase in each component. Standard deviations are in parentheses. 


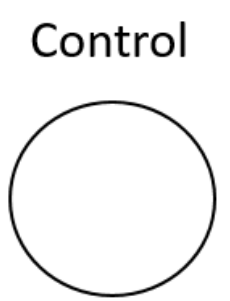

EXT

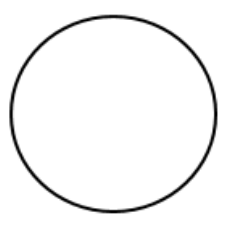

EXT

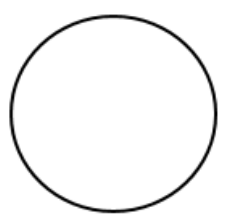

EXT

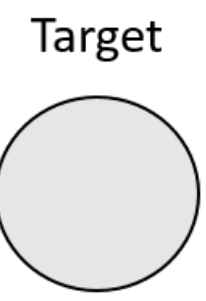

VTVDRO

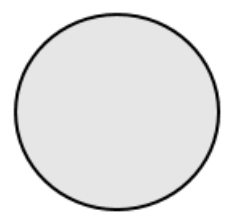

EXT

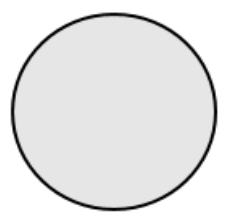

EXT
Alternative

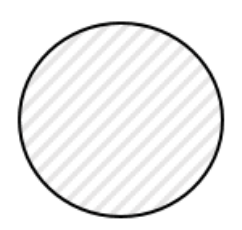

VI
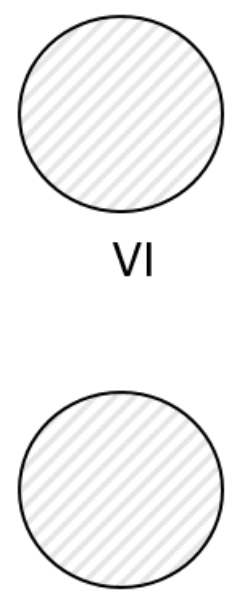

EXT
DRO Training

VI EXT Alternative Reinforcement

Resurgence Test

Figure 1. Diagram of the procedure for Experiment 1. The schedule abbreviations at the bottom of each circle indicate the schedule in effect for that key. 


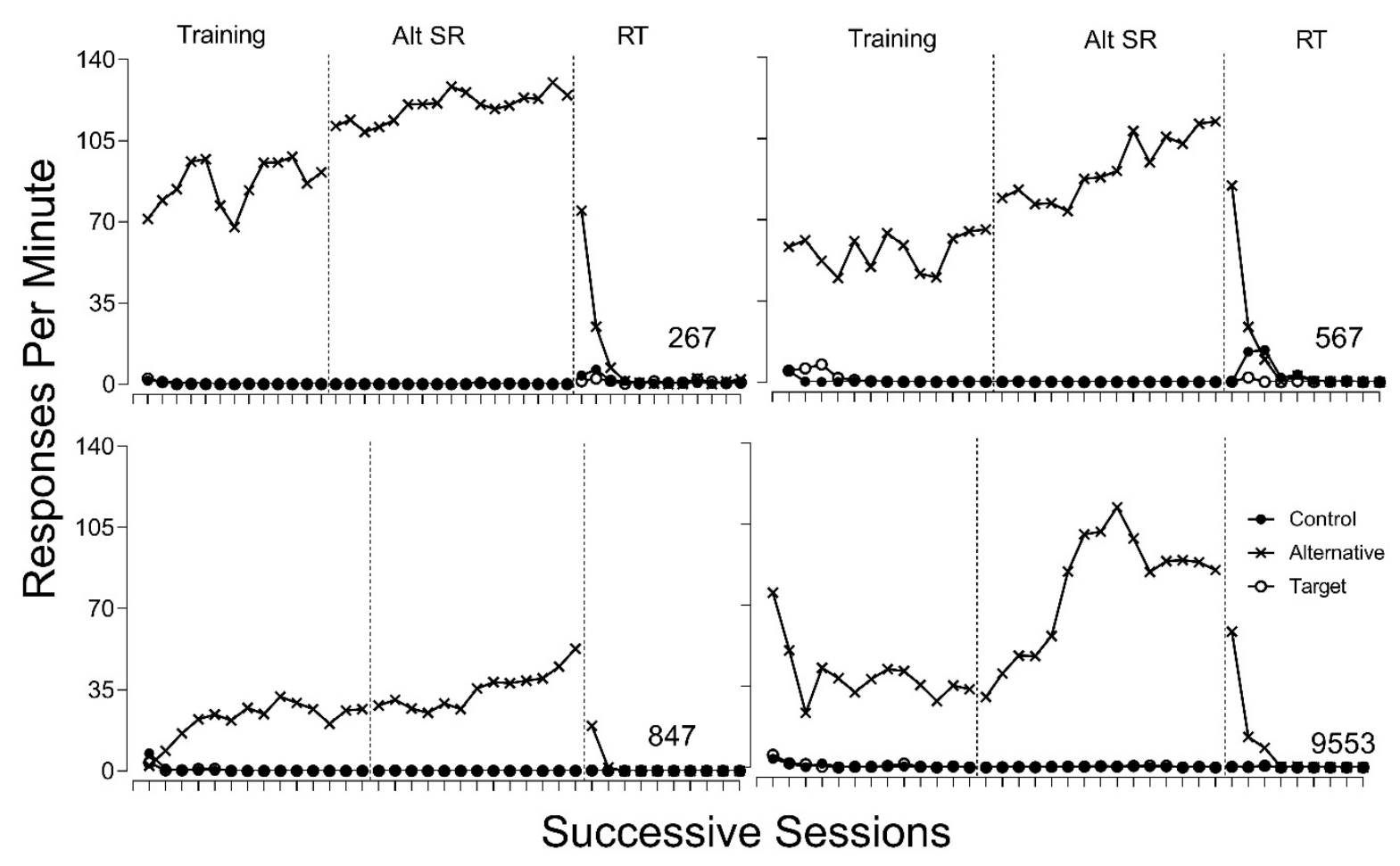

Figure 2. Response rates on the Alternative, Target, and Control keys during each phase of Experiment 1. 

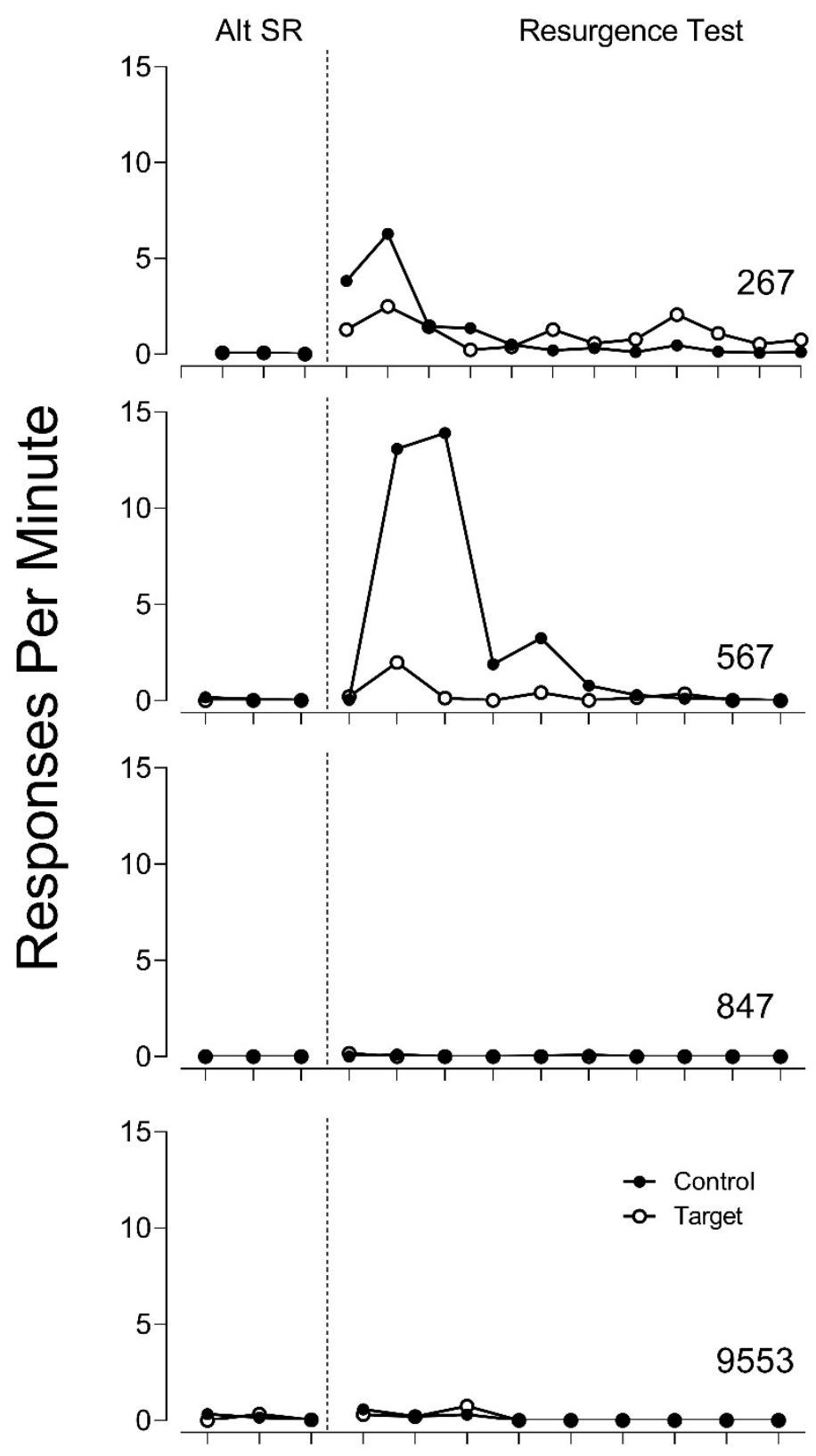

Successive Sessions

Figure 3. Control and Target Response rates for the last three sessions of the Alternativereinforcement phase and all sessions of the RT phase for all pigeons in Experiment 1. 


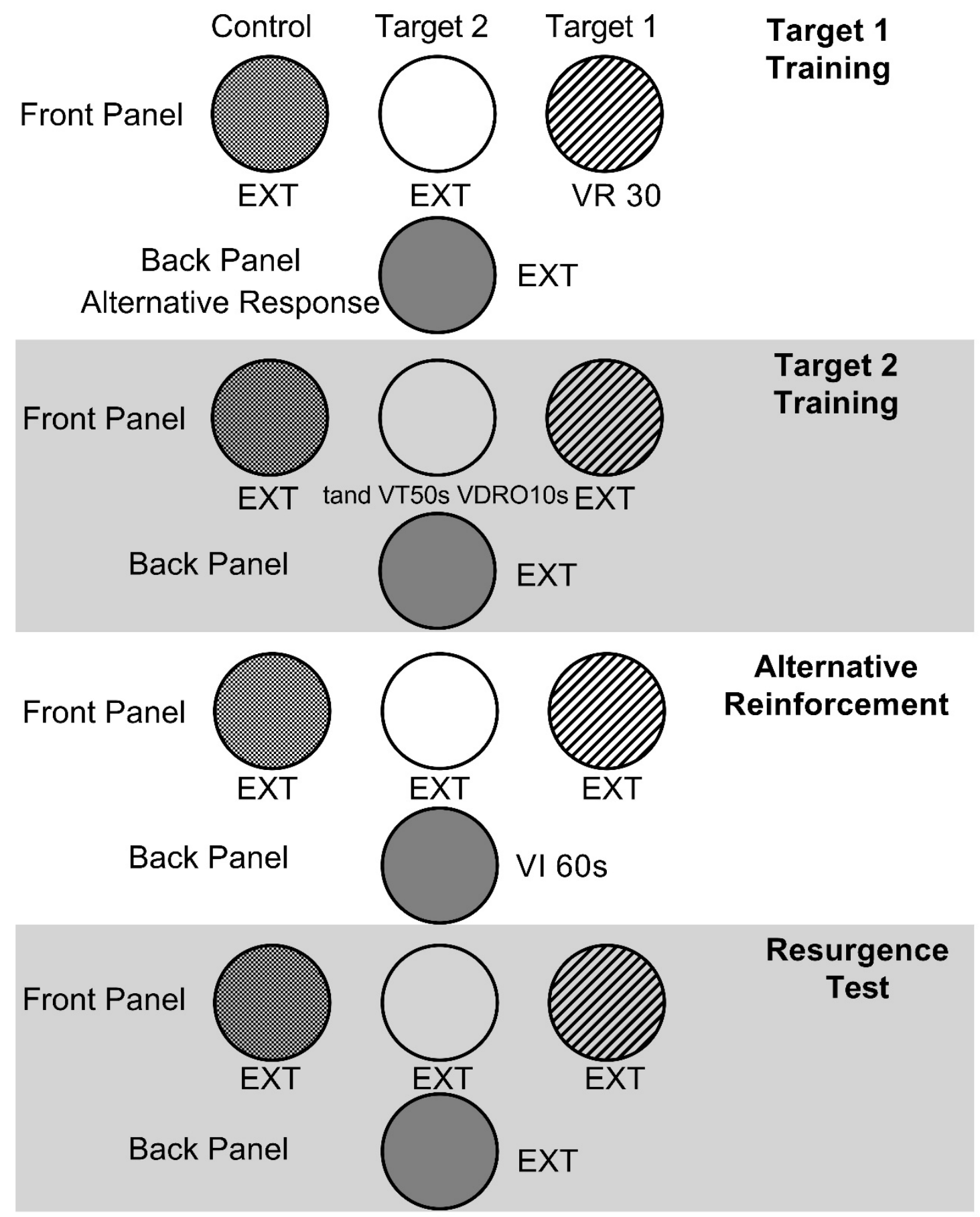

Figure 4. Diagram of the procedure for Experiment 2a. The schedule abbreviations at the bottom of each circle indicate the schedule of reinforcement in effect for that key. 


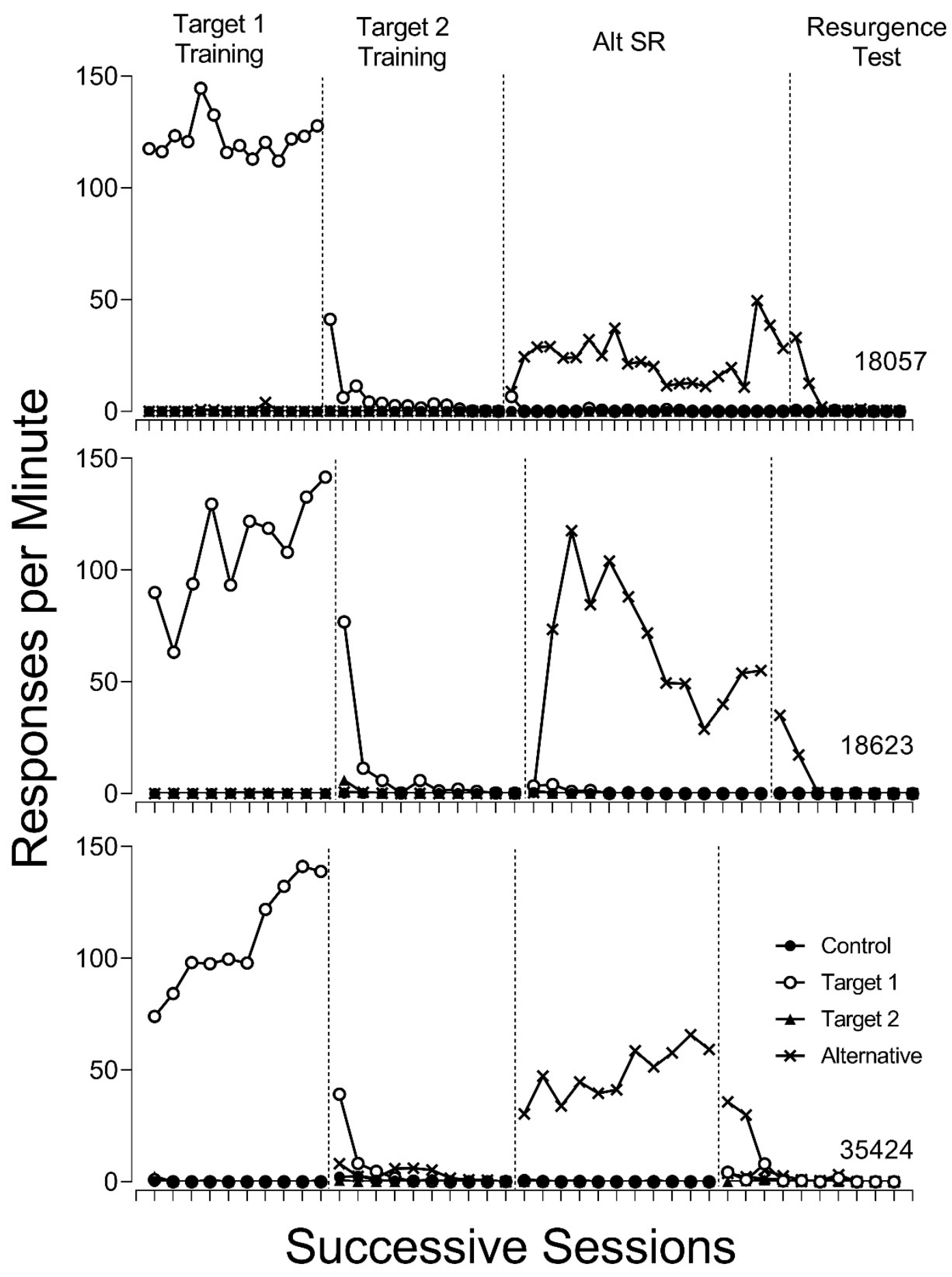

Figure 5. Responses per minute of the Alternative, Target 1, Target 2, and Control keys for all pigeons in Experiment 2a. 


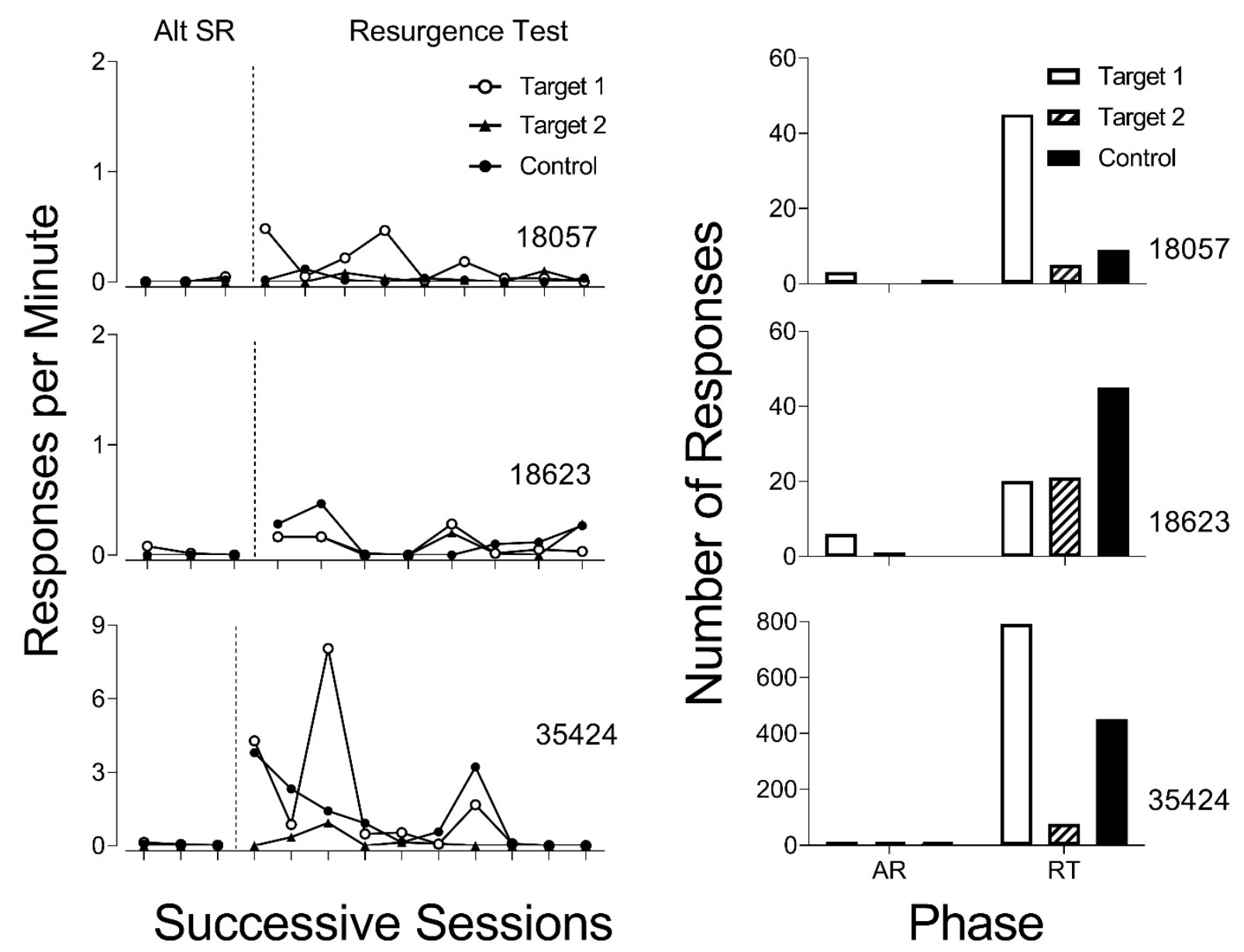

Figure 6. Left graphs: Experiment 2a responses per minute for each pigeon during the last three sessions of the Alternative-Reinforcement phase and all sessions for the Resurgence-Test phase. Right graphs: Total responses (key pecks) on the Target 1, Target 2, and Control keys during the last three sessions of the Alternative-Reinforcement phase and first three days of the ResurgenceTest phase. Note the different y-axis scale for both graphs for Pigeon 35424. 


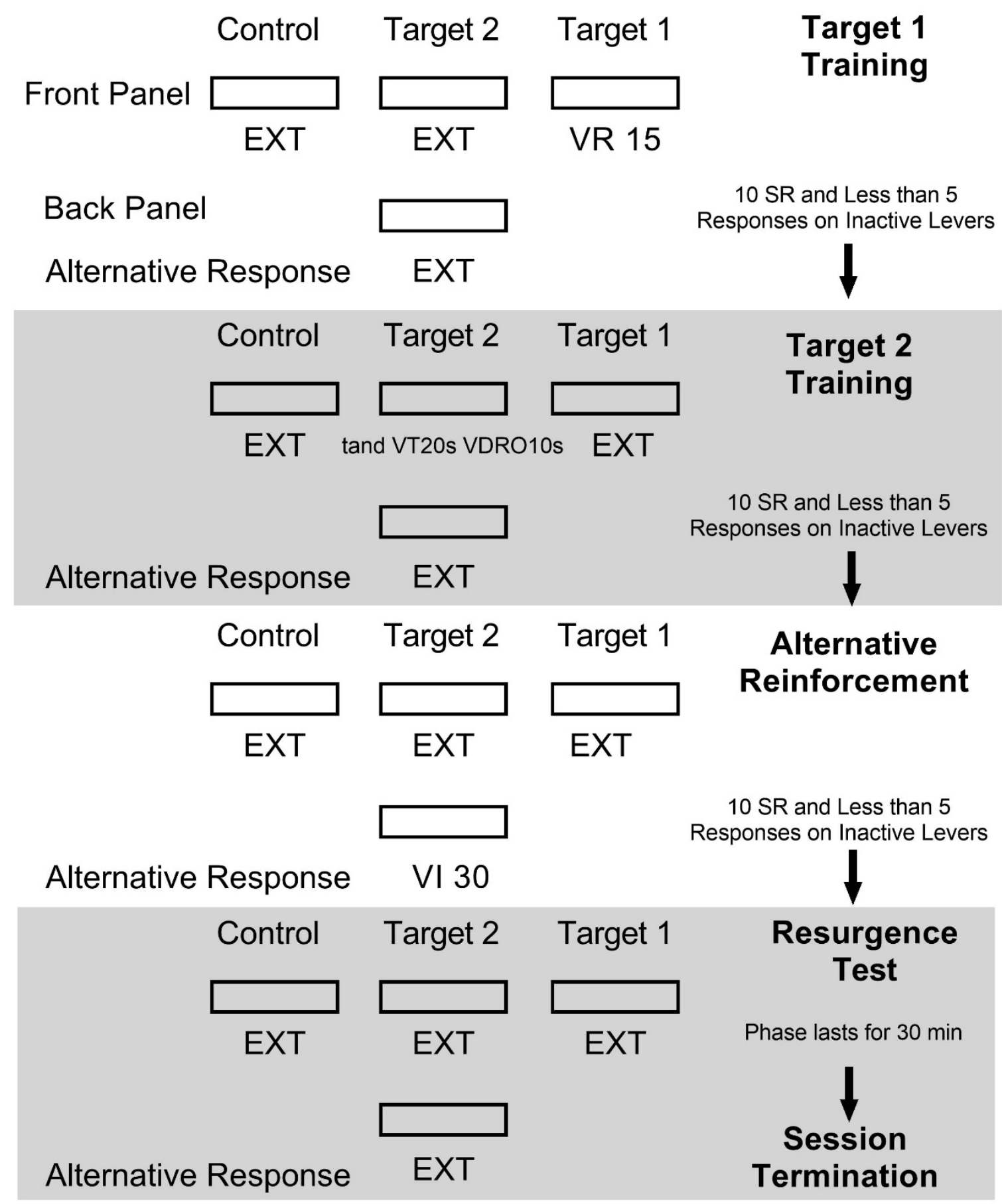

Figure 7. Diagram of the procedure used in Experiment 2b. The schedule abbreviations at the bottom of each rectangle indicate the schedule in effect for that lever. Sessions terminated following the 30-min RT phase. 


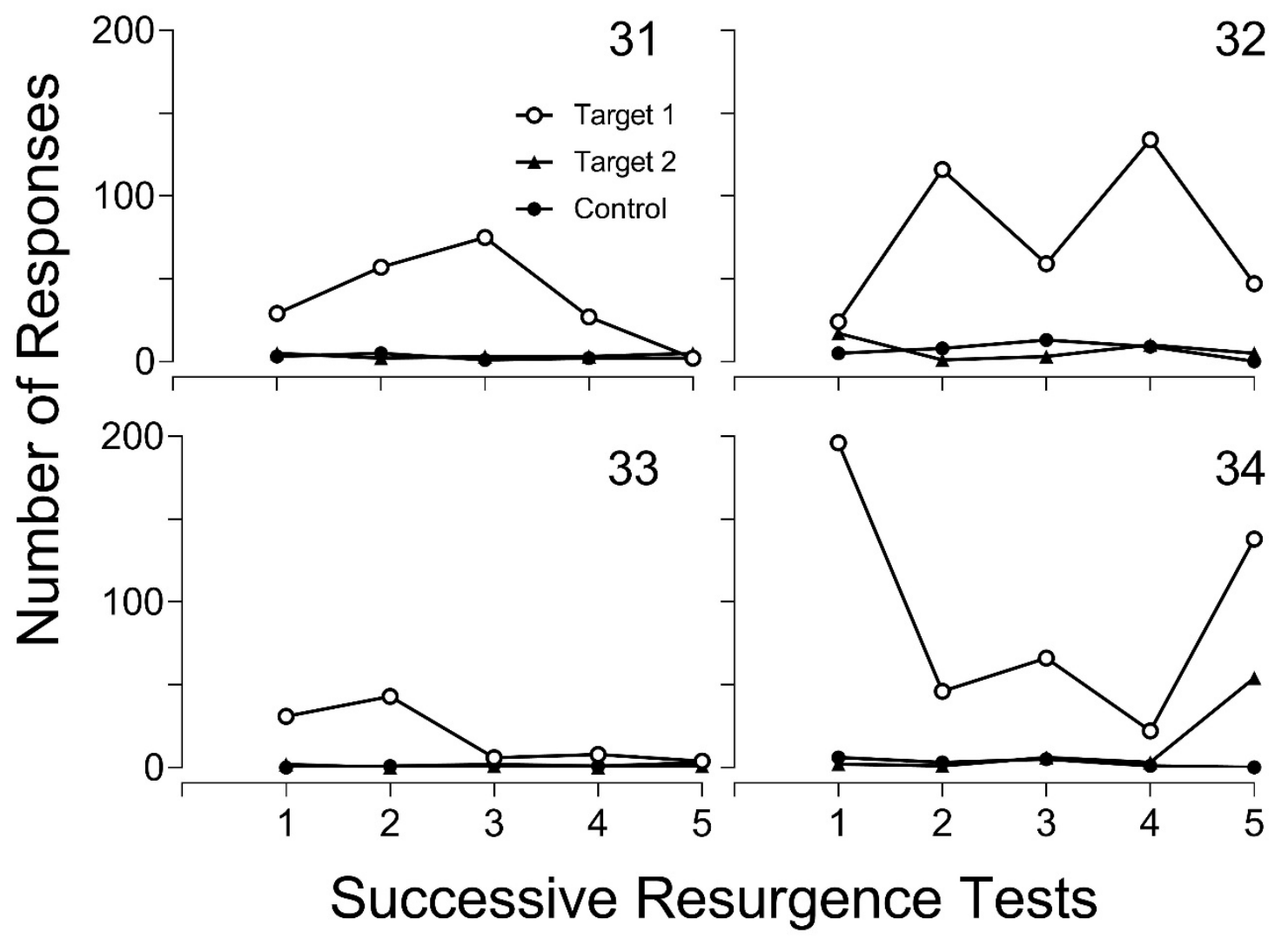

Figure 8. Number of Target 1, Target 2, and Control responses during the RT phase for each rat in Experiment 2b. 


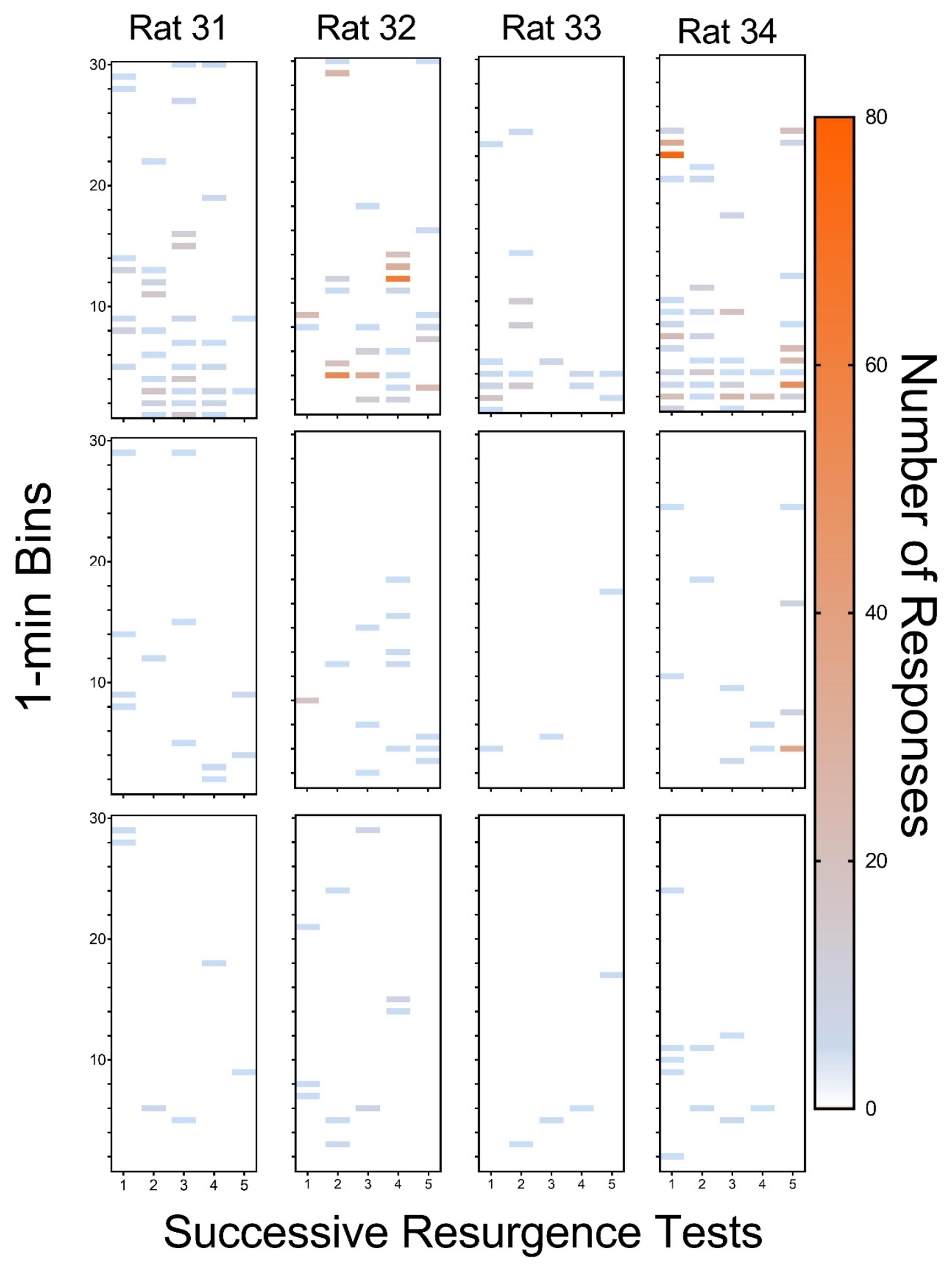

Figure 9. Frequency of Target 1 (top), Target 2 (middle), Control (bottom) responses during successive 1-min bins of the RT phase of each experimental session of Experiment 2b. Orange rectangles indicate a higher frequency of responding, whereas blue rectangles indicate a lower frequency of responding. 


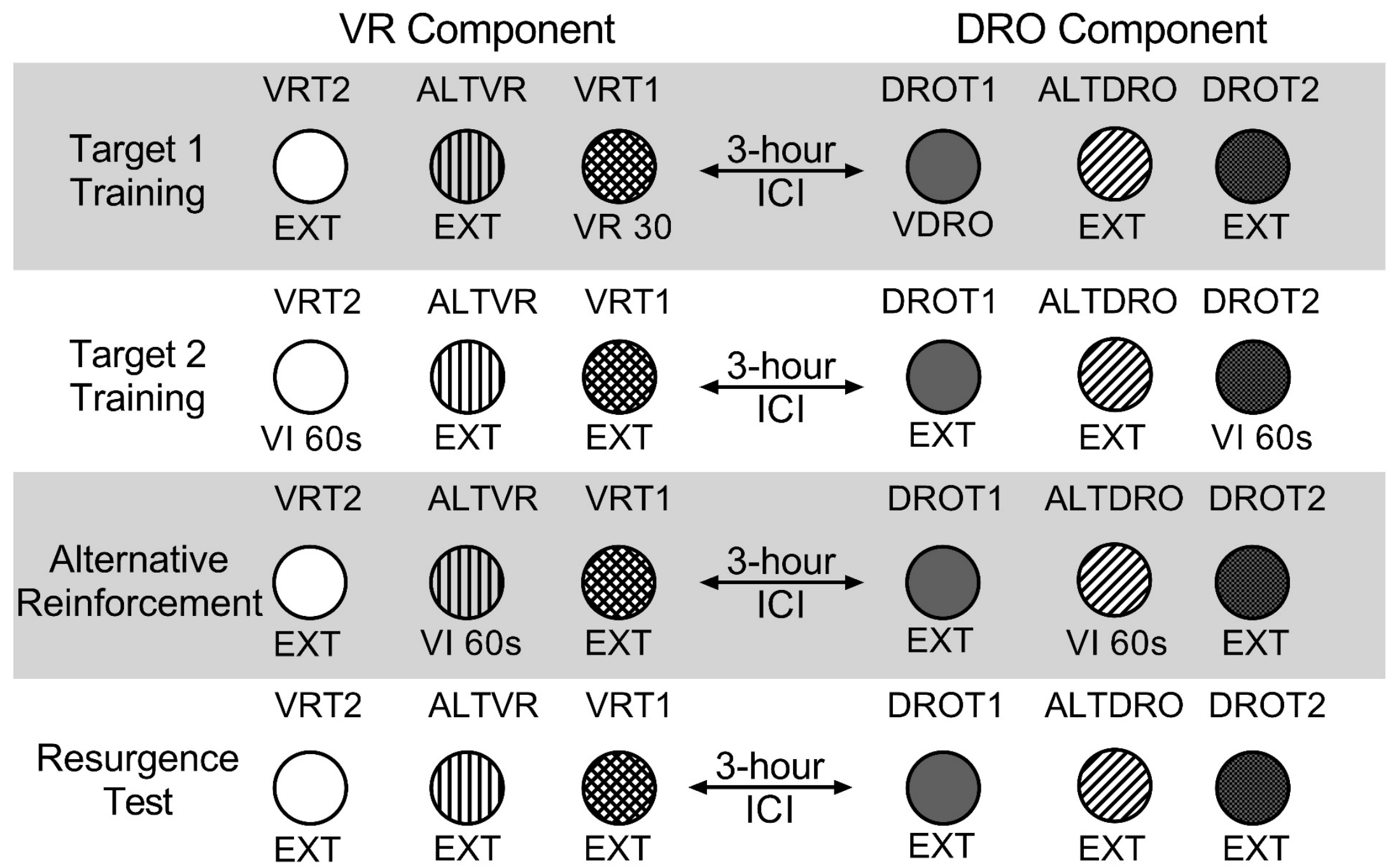

Figure 10. Diagram of the procedure used in Experiment 3. The schedule abbreviations at the bottom of each circle indicate the schedule in effect for that key. Each component was separated by a 3-hour intercomponent interval and sessions terminated after 60 reinforcer deliveries or 75-min had elapsed. 


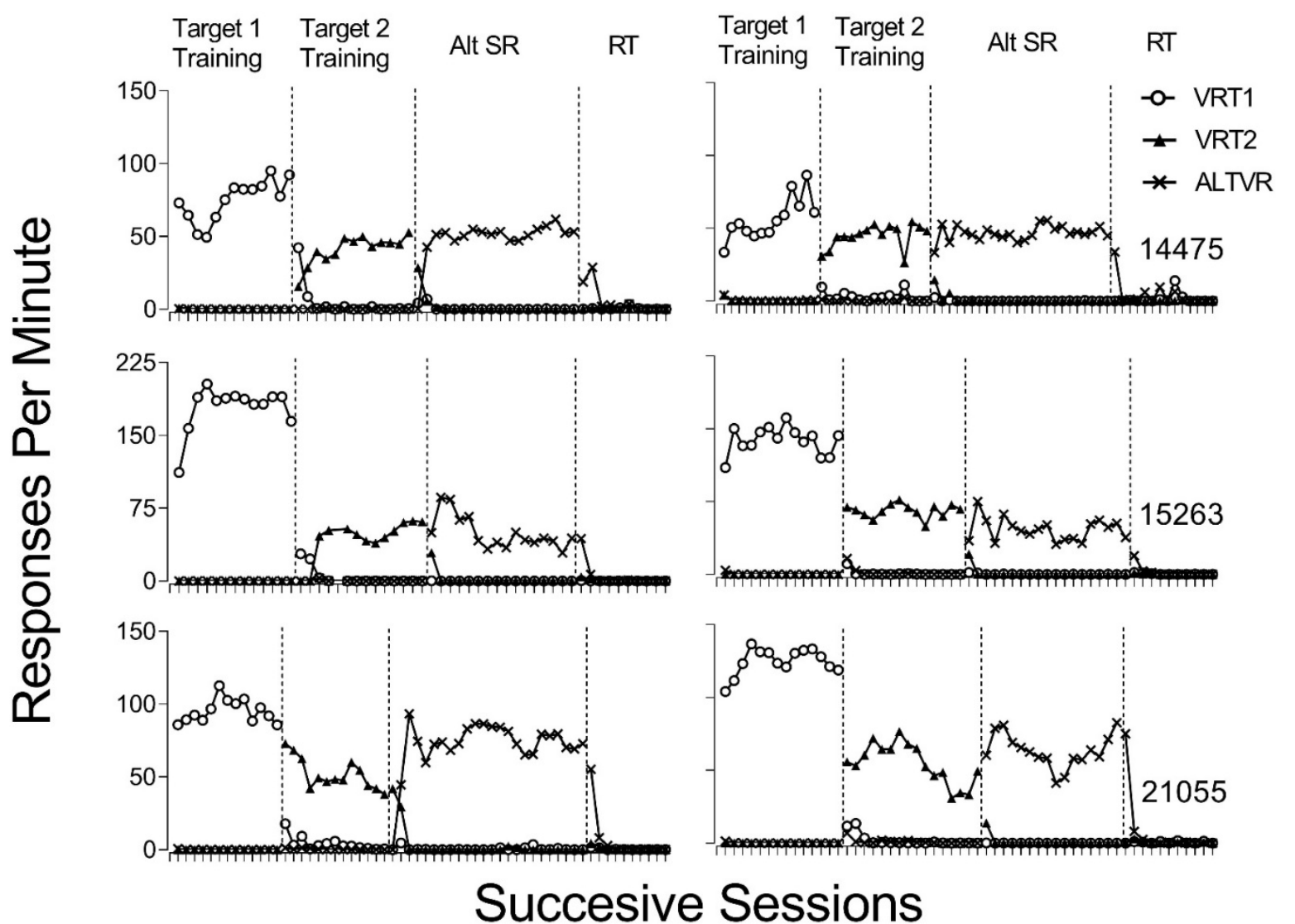

Figure 11. Rates of the VRT1, VRT2, and ALT VR responses during each phase for each pigeon in Experiment 3. The data are from the VR component. The left panel is from the first iteration of the procedure and the right is from the replication. Note the different $y$-axis scale for Pigeon 15263. 


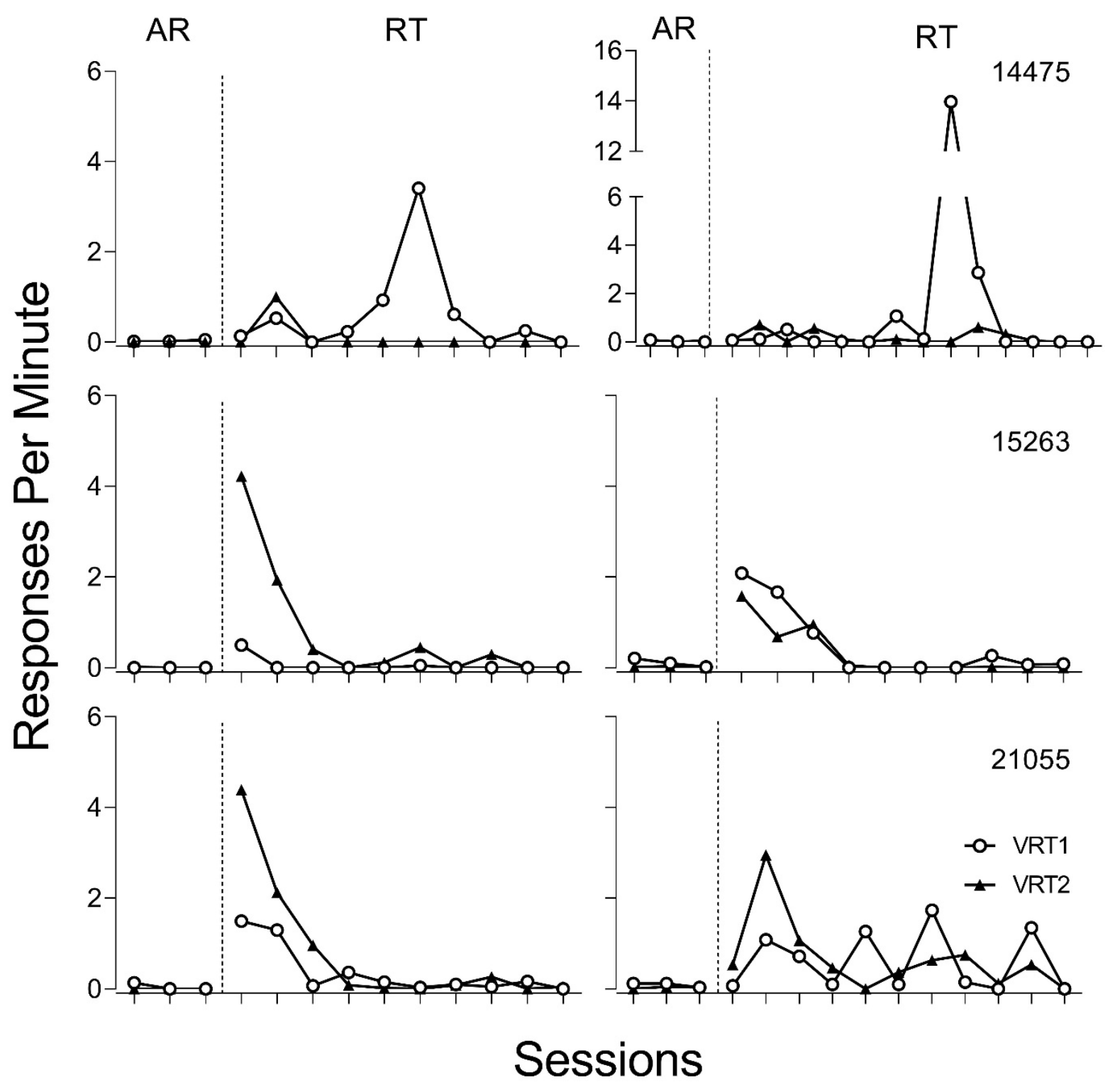

Figure 12. Response rates of VRT1 and VRT2 during the last three sessions of the AR phase and the RT phase for each pigeon in Experiment 3. The left is from the first iteration of the procedure and the right is from the replication. Note the different y-axis scale for Pigeon 14475. 


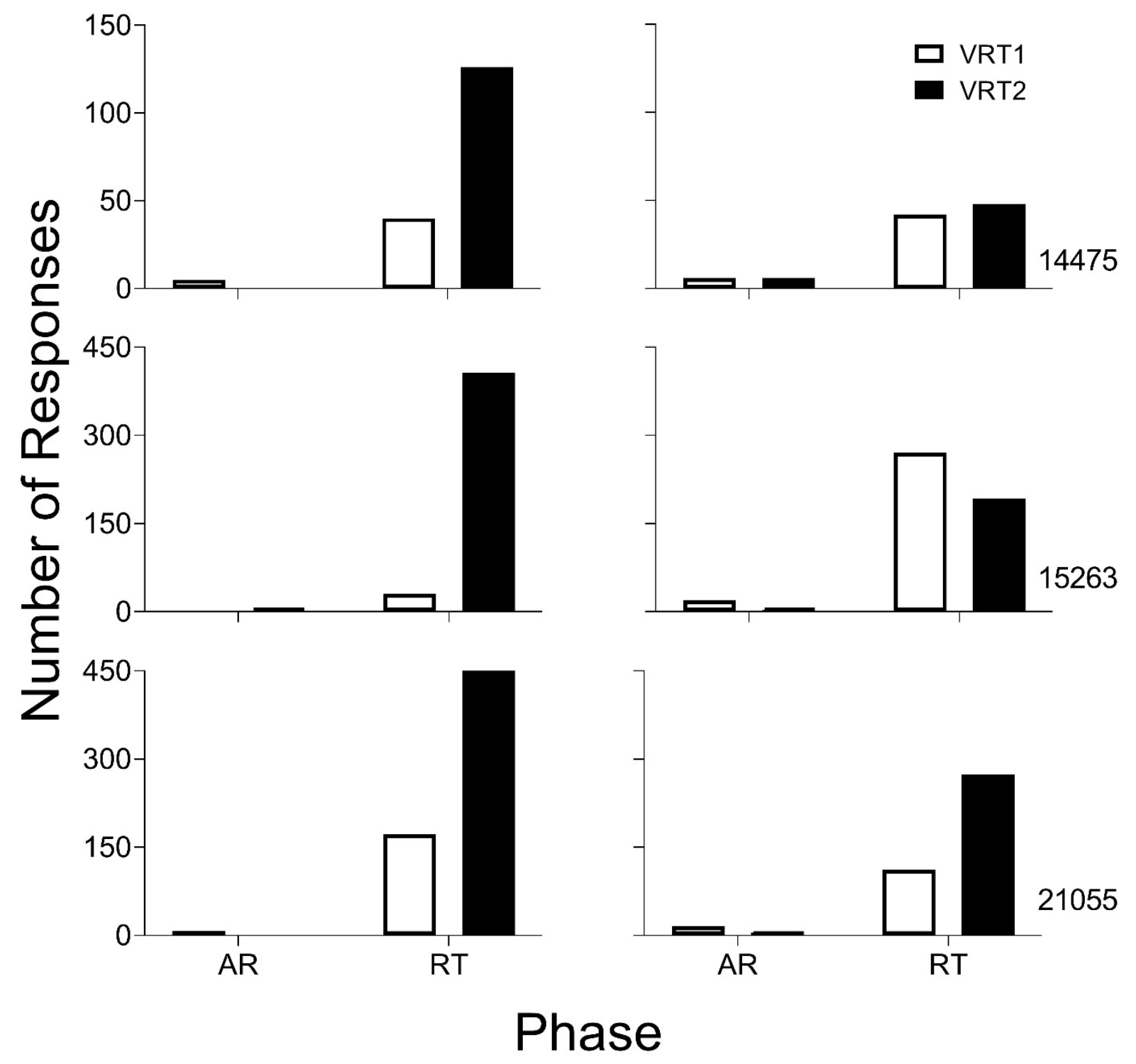

Figure 13. Total responses (key pecks) on the VRT1 and VRT2 keys during the last three sessions of the Alternative-Reinforcement phase and first three days of the Resurgence-Test phase for each pigeon in Experiment 3. The graphs on the left are from the first RT phase and the graphs on the right are from the second RT phase. Note the different y-axis scale for Pigeon 14475. 


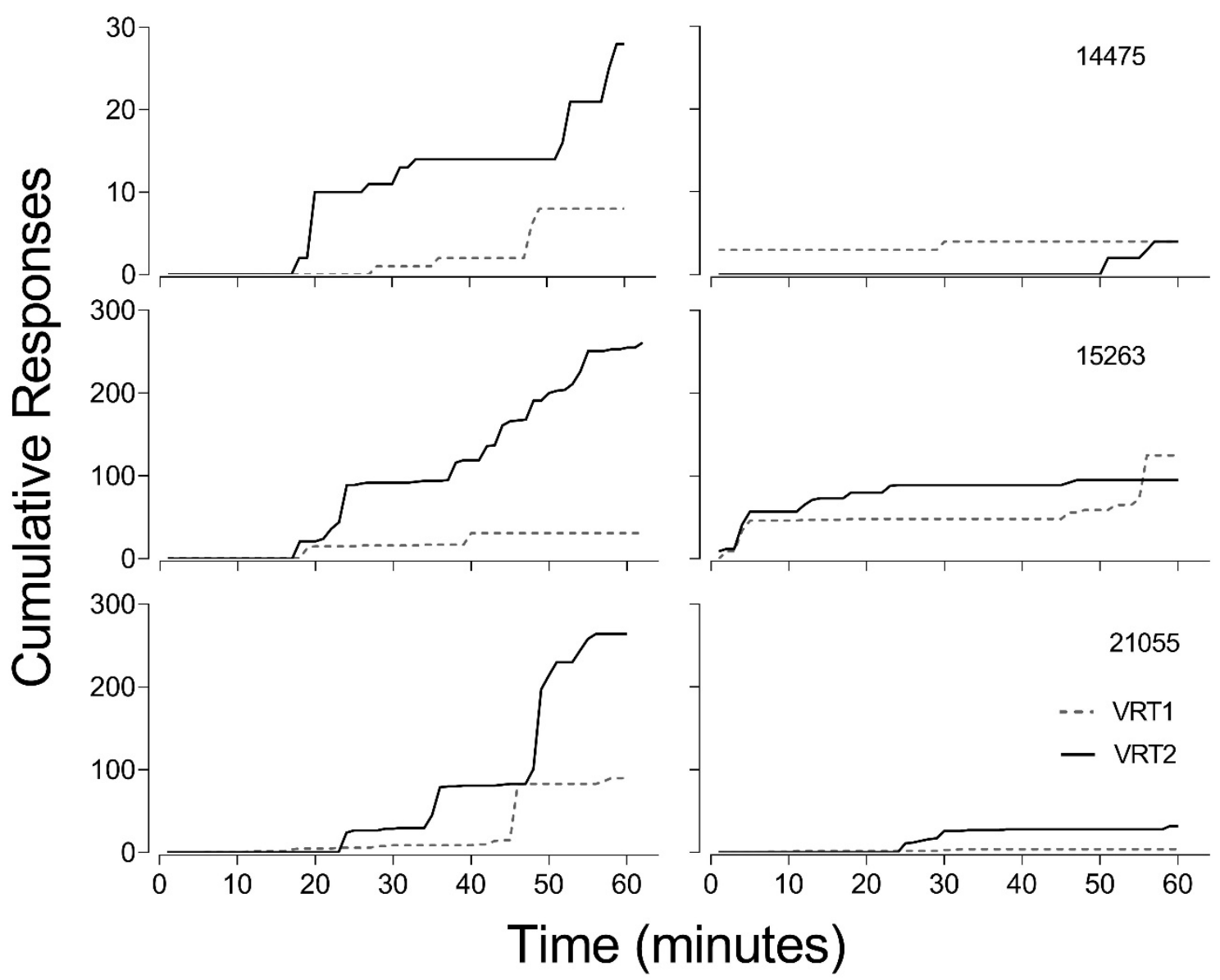

Figure 14. Cumulative record of VRT1 and VRT2 responses during the first session of the first RT phase (left panel) and the first session of the replication (right panel) for each pigeon in Experiment 3. Note the different y-axis scale for Pigeon 14475. 


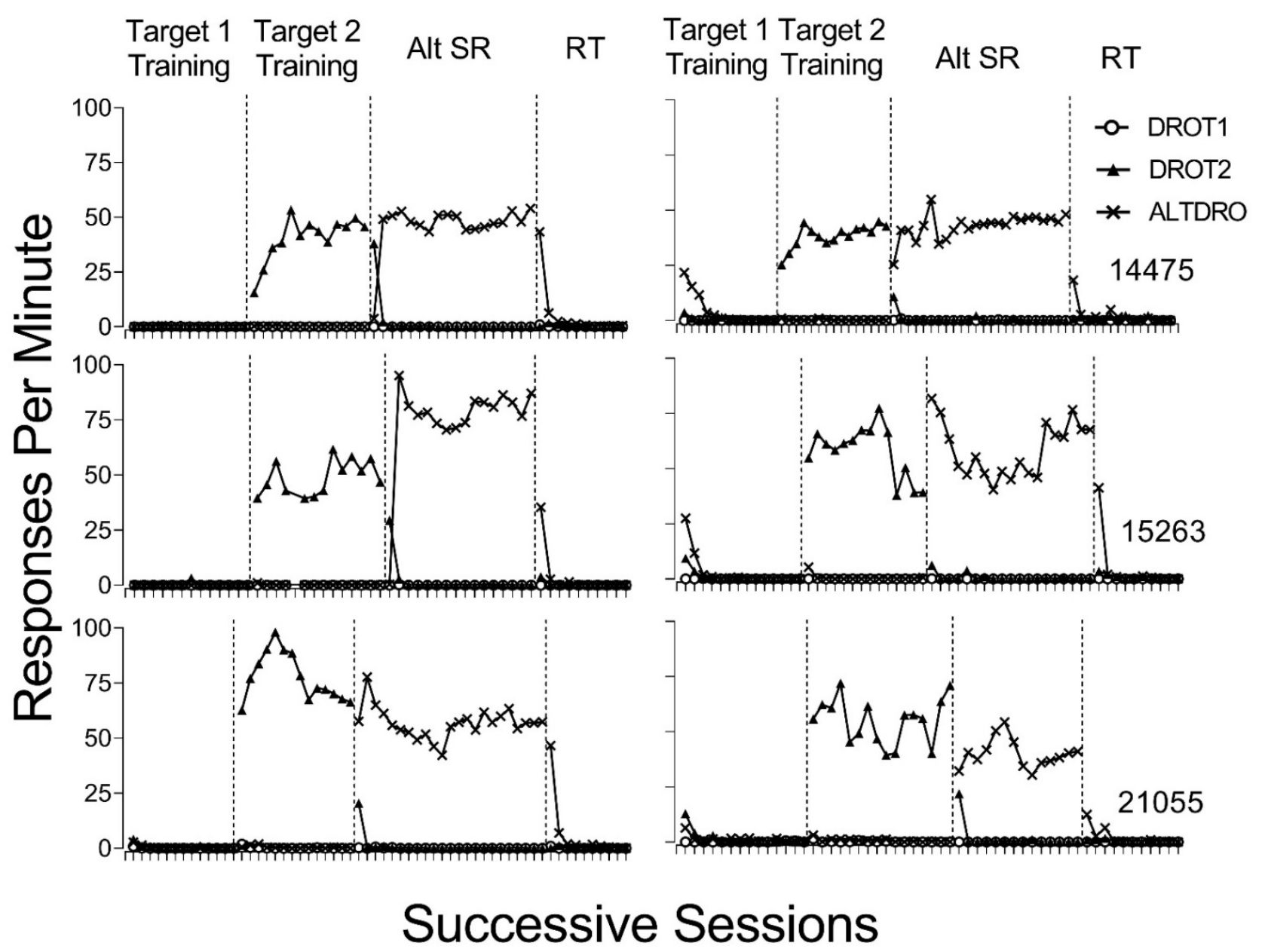

Figure 15. Response rates of the DROT1, DROT2, and ALDRO for each phase for each pigeon in Experiment 3. The data presented in this figure are the DRO Component. The left panel is from the first iteration of the procedure and the right is from the replication. 


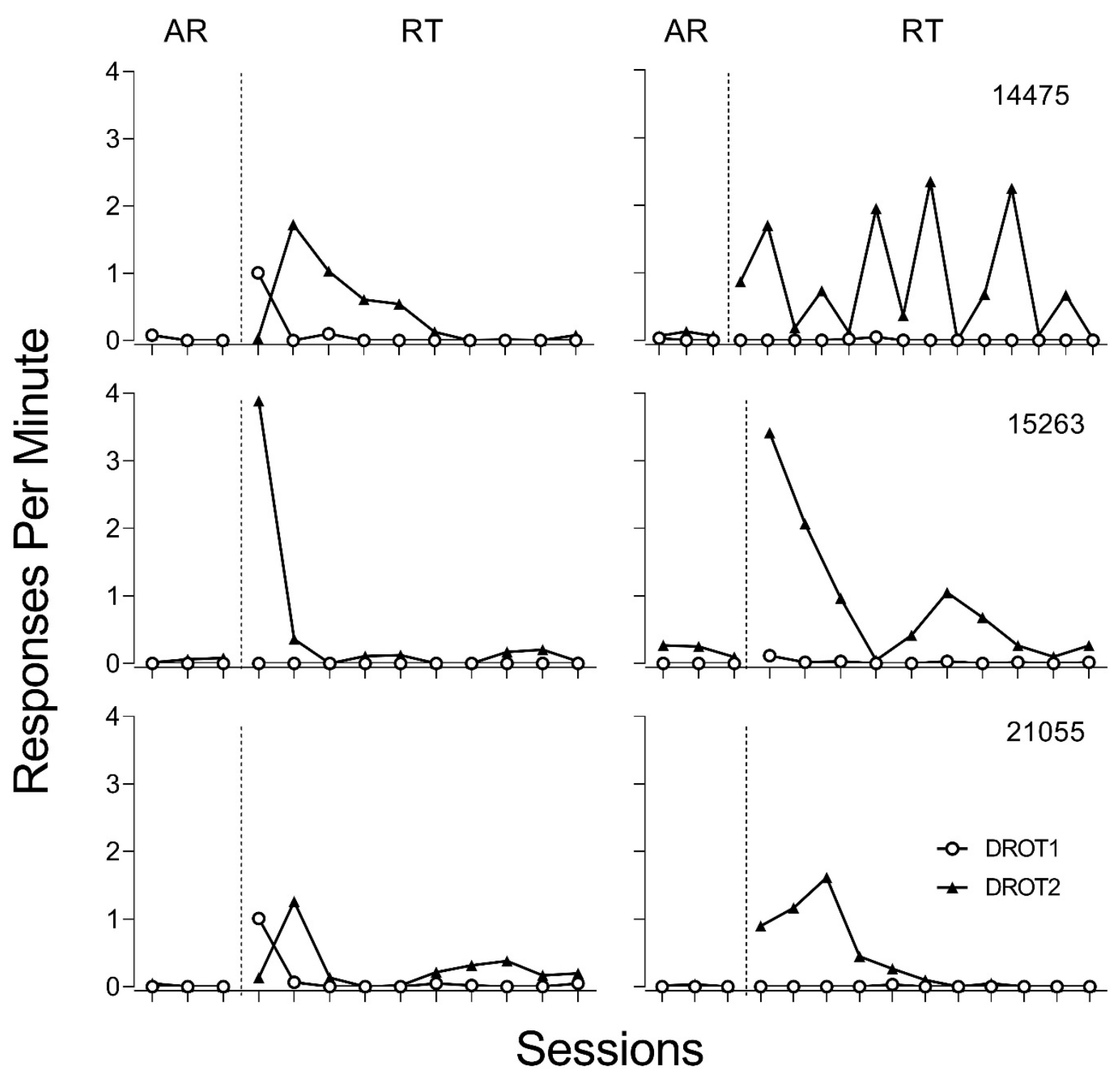

Figure 16. Response rates of DROT1 and DROT2 during the last three sessions of the AR phase and the RT phase for each pigeon in Experiment 3. The left is from the first iteration of the procedure and the right is from the replication. 


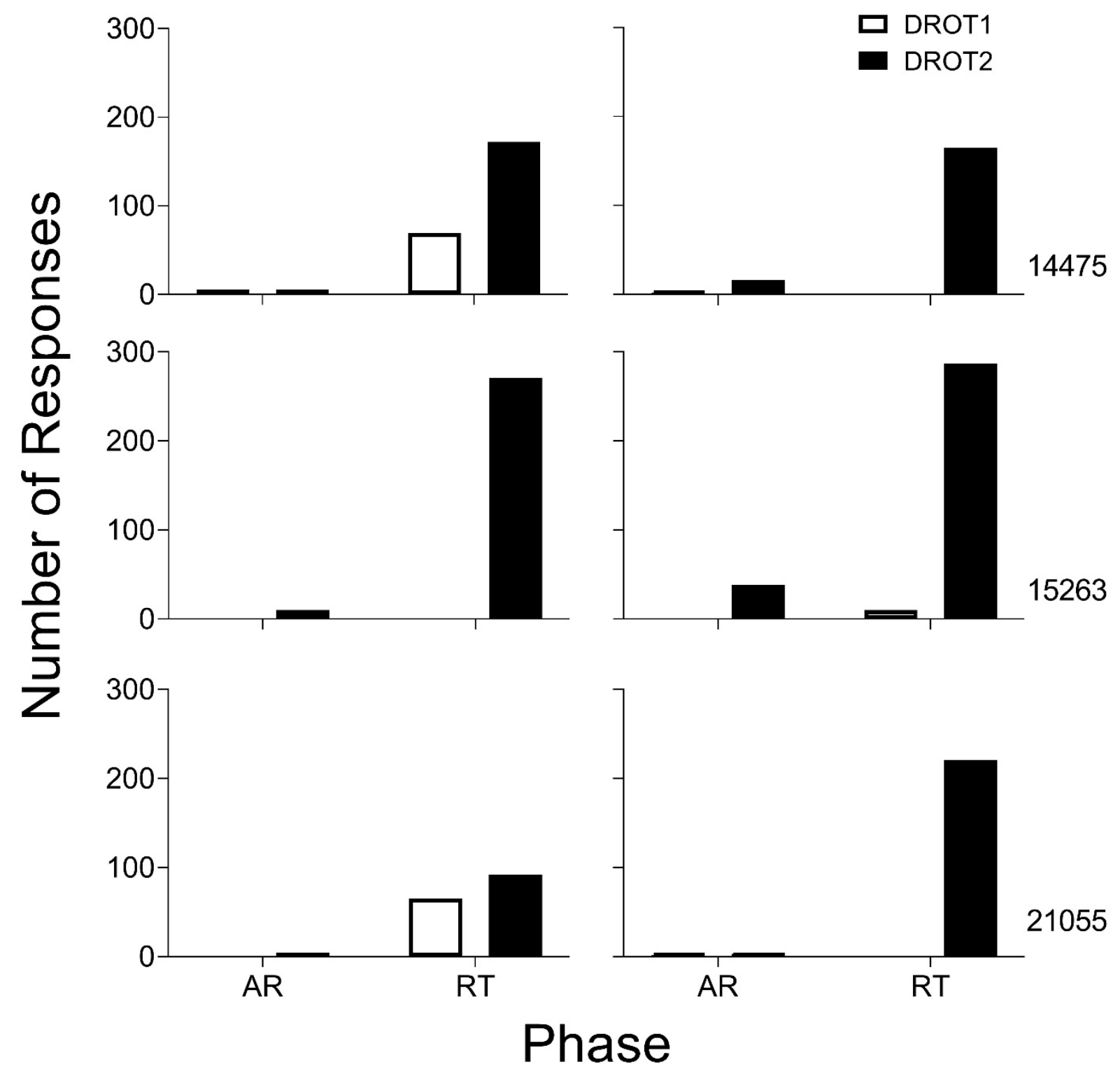

Figure 17. Total responses (key pecks) on the DROT1 and DROT2 keys during the last three sessions of the Alternative-Reinforcement phase and first three days of the Resurgence-Test phase for each pigeon in Experiment 3. The graphs on the left are from the first RT phase and the graphs on the right are from the second RT phase. 


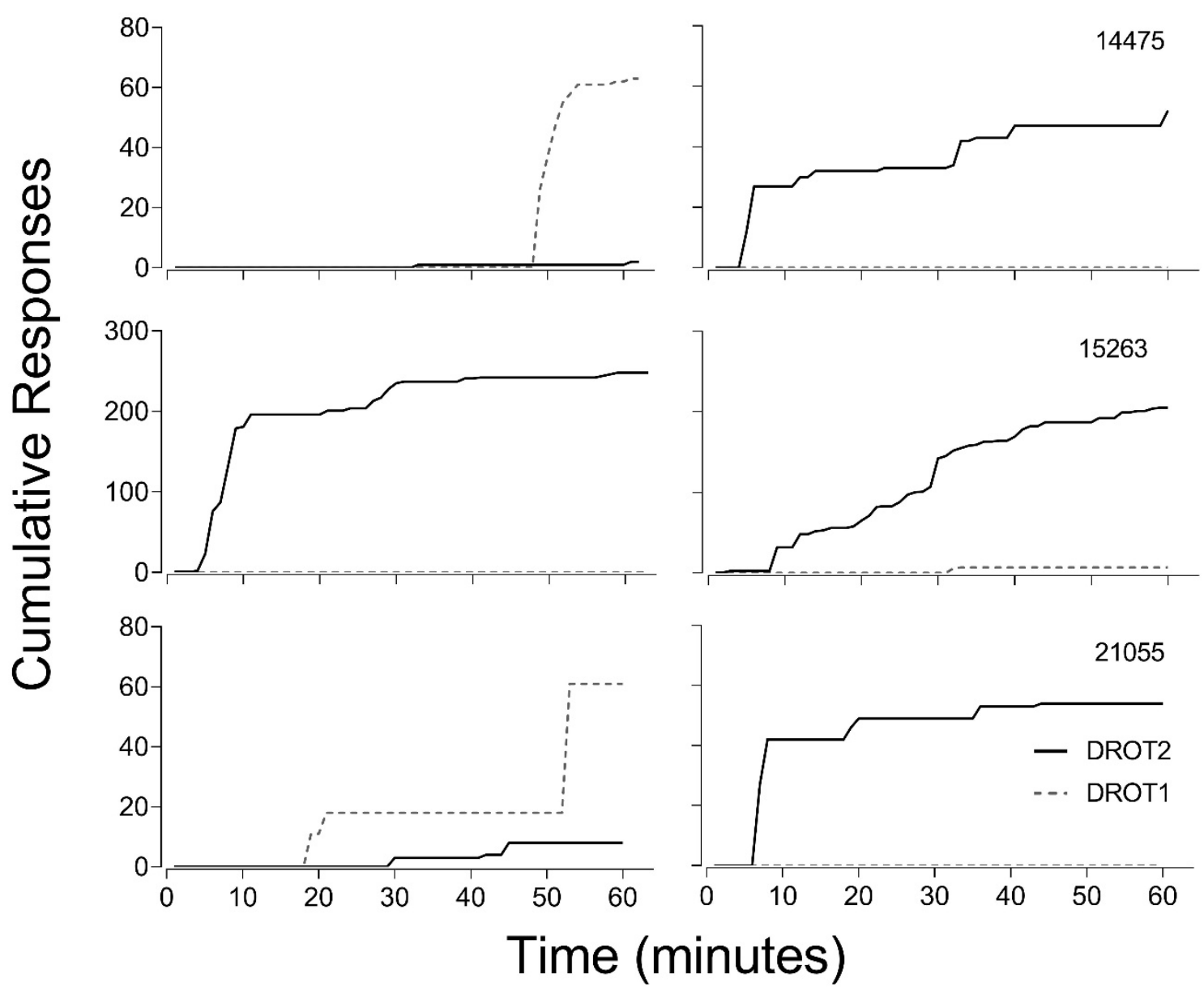

Figure 18. Cumulative record of DROT1 and DROT2 responses during the first session of the first RT phase (left panel) and the first session of the replication (right panel) for each pigeon in Experiment 3. Note the different y-axis scale for Pigeon 15263. 


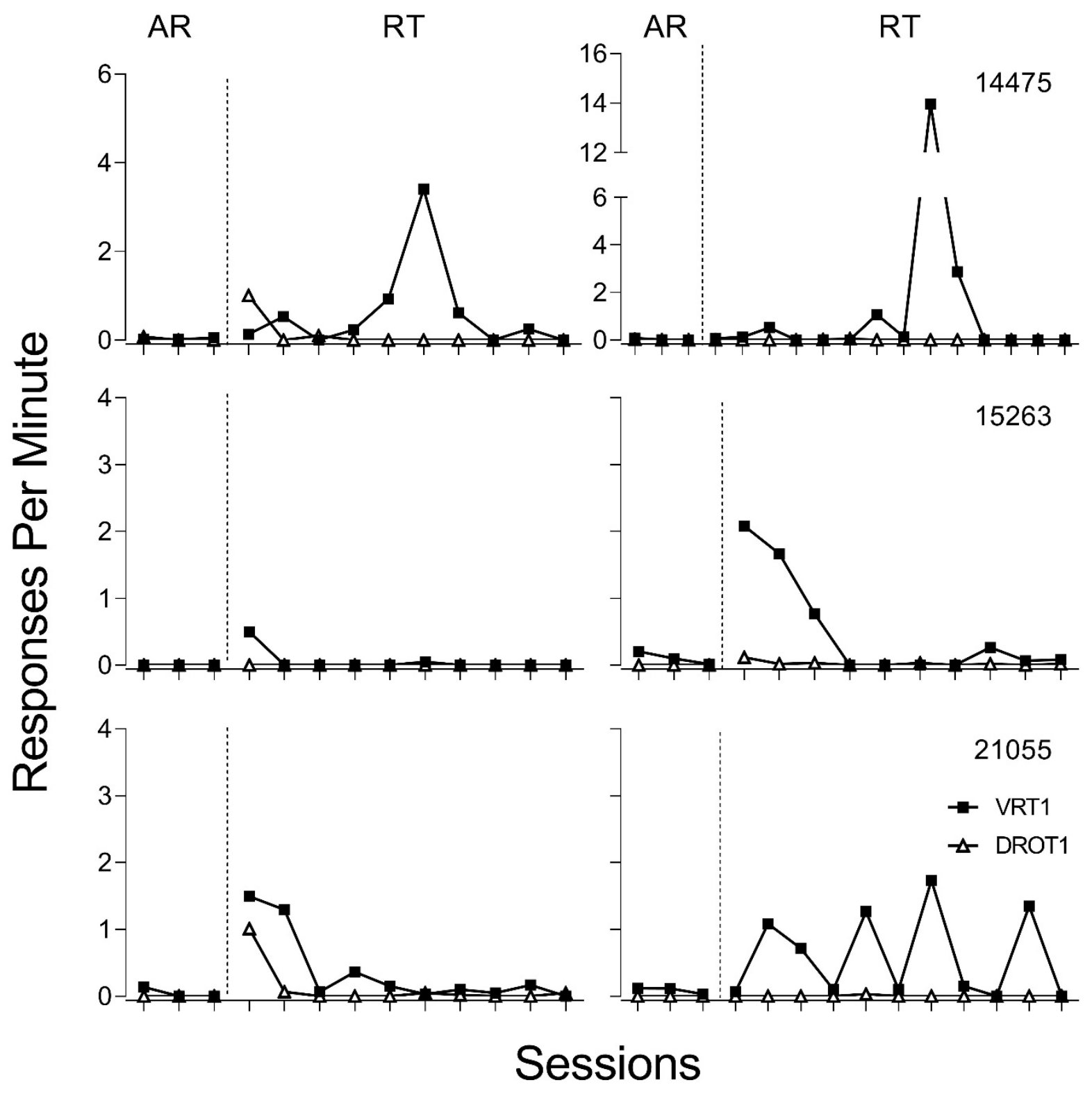

Figure 19. Response rates of DROT1 and VRT1 during the last three sessions of the AR phase and the RT phase for each pigeon in Experiment 3. The left is from the first iteration of the procedure and the right is from the replication. Note the different y-axis scale for Pigeon 14475 


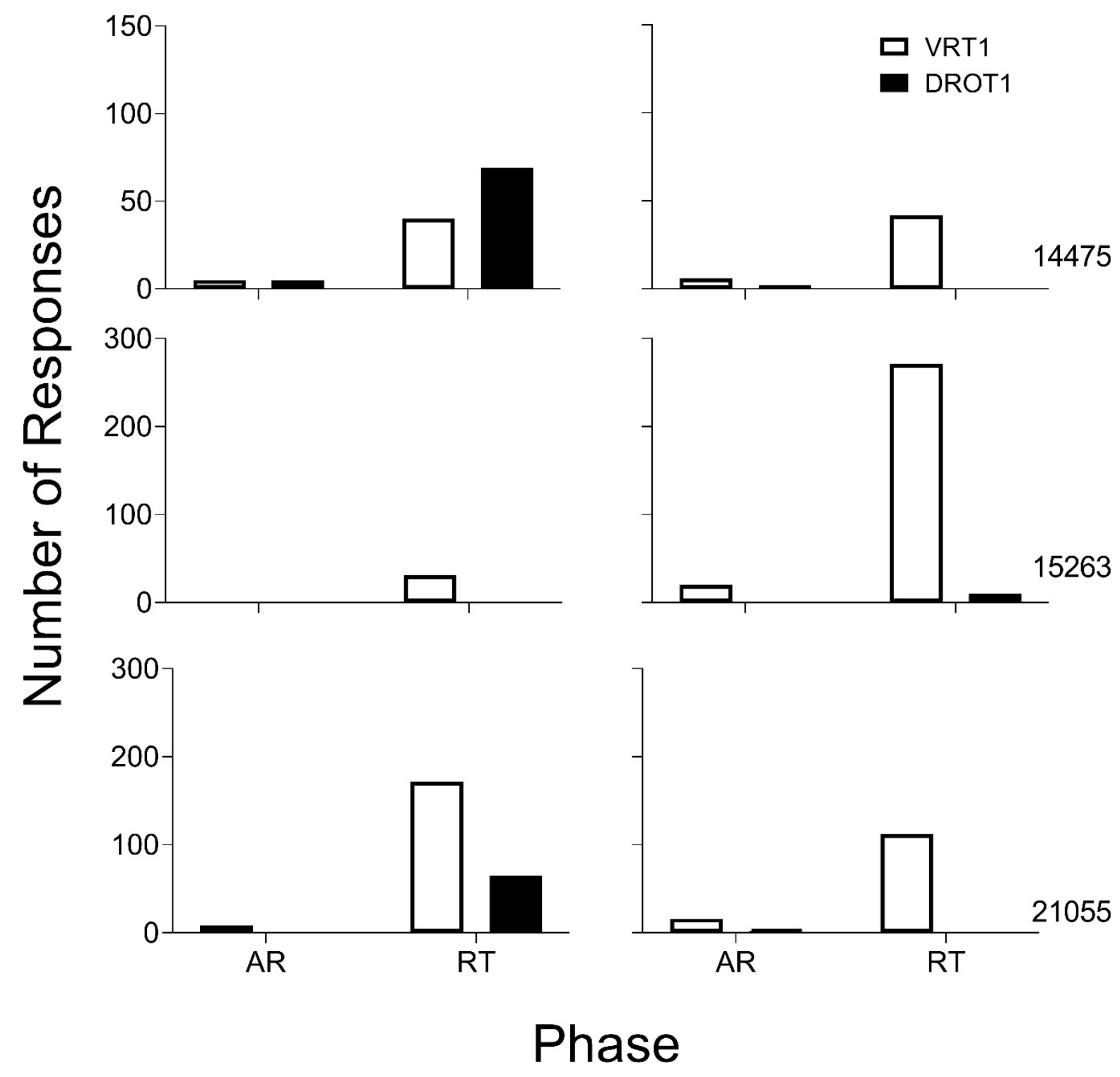

Figure 20. Total responses (key pecks) on the DROT1 and VRT1 keys during the last three sessions of the Alternative-Reinforcement phase and first three days of the Resurgence-Test phase for each pigeon in Experiment 3. The graphs on the left are from the first RT phase and the graphs on the right are from the second RT phase. Note the different y-axis scale for Pigeon 14475. 


\section{Appendix A \\ Glossary of Terms}

Alternative Response: Response reinforced in the phase that proceeds the Resurgence Test phase. In all experiments this is the response maintained with a VI schedule of reinforcement during the second (Experiment 1) and third phases of Experiments 2a, 2b, and 3.

ALTDRO: Alternative response of the DRO history sessions in Experiment 3

ALTVR: Alternative response for the VR History sessions. in Experiment 3

Control Response: Response with no within-experiment reinforcement history. Control responses are included in Experiments 1, 2a, and 2b.

DROT1: Target 1 response maintained with the differential reinforcement of other behavior (DRO) schedule in the DRO Component of Experiment 3

DROT2: Target 2 response for the DRO Component in Experiment 3

Target: Response trained during the first phase of a three-phase resurgence procedure. This is the to be-resurged response in Experiment 1.

Target 1: First trained response in a resurgence procedure. This is the response with the VR history of reinforcement in Experiment 2a and 2b.

Target 2: Second trained response in a resurgence procedure. This is the DRO response reinforced during the second phase of Experiment 2a and 2b.

VRT1: Target 1 response maintained with the variable ratio (VR) schedule of reinforcement in Experiment 3. T1VR responses occurred during the VR Component.

VRT2: Target 2 response for the VR Component in Experiment 3 
Appendix B

Supplemental Figures for Experiment 2b
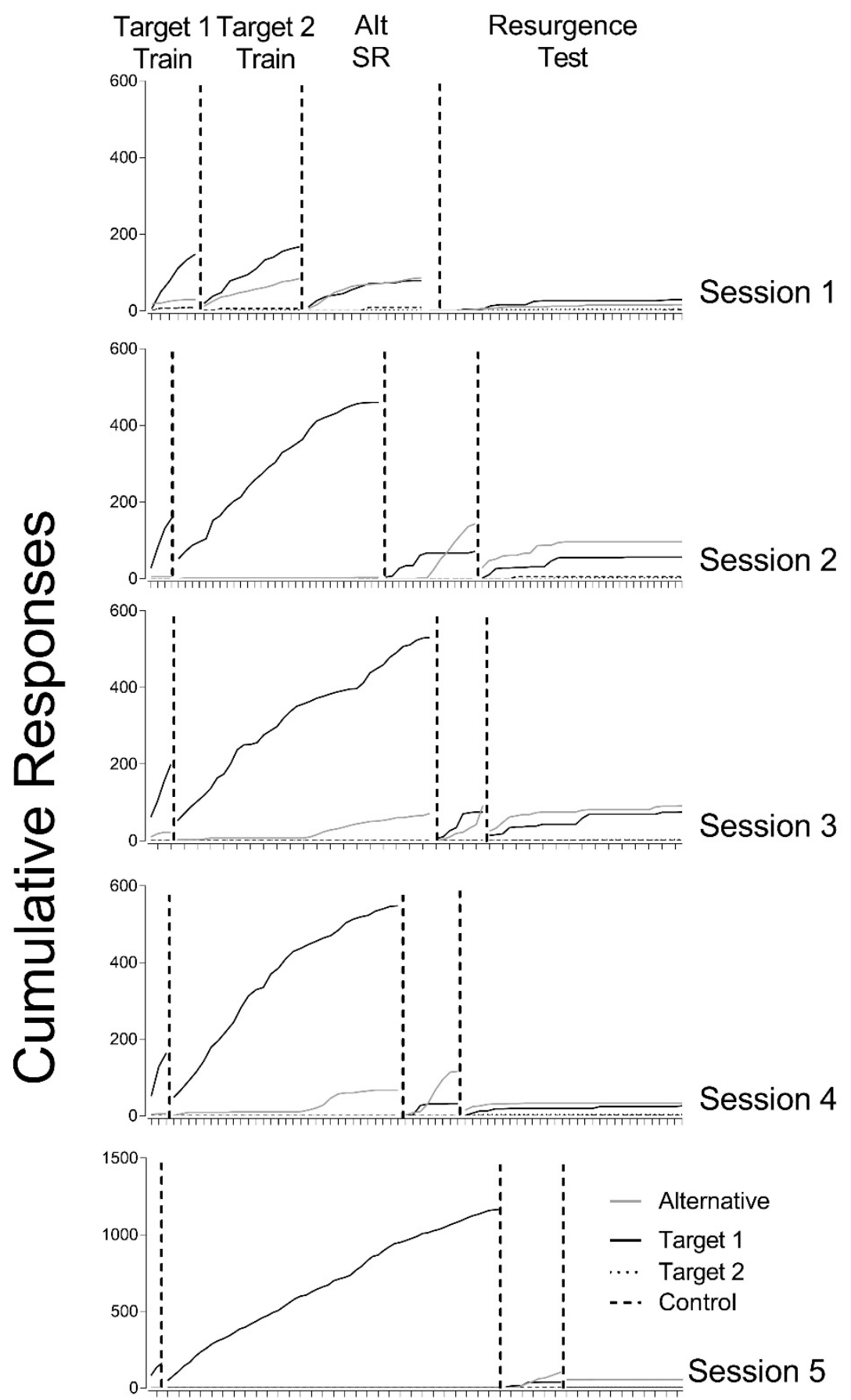

\section{Time (minutes)}

Figure B1. Cumulative record of Alternative, Target-1, Target-2, and Control responses during each session of Experiment $2 b$ for Rat 31. 


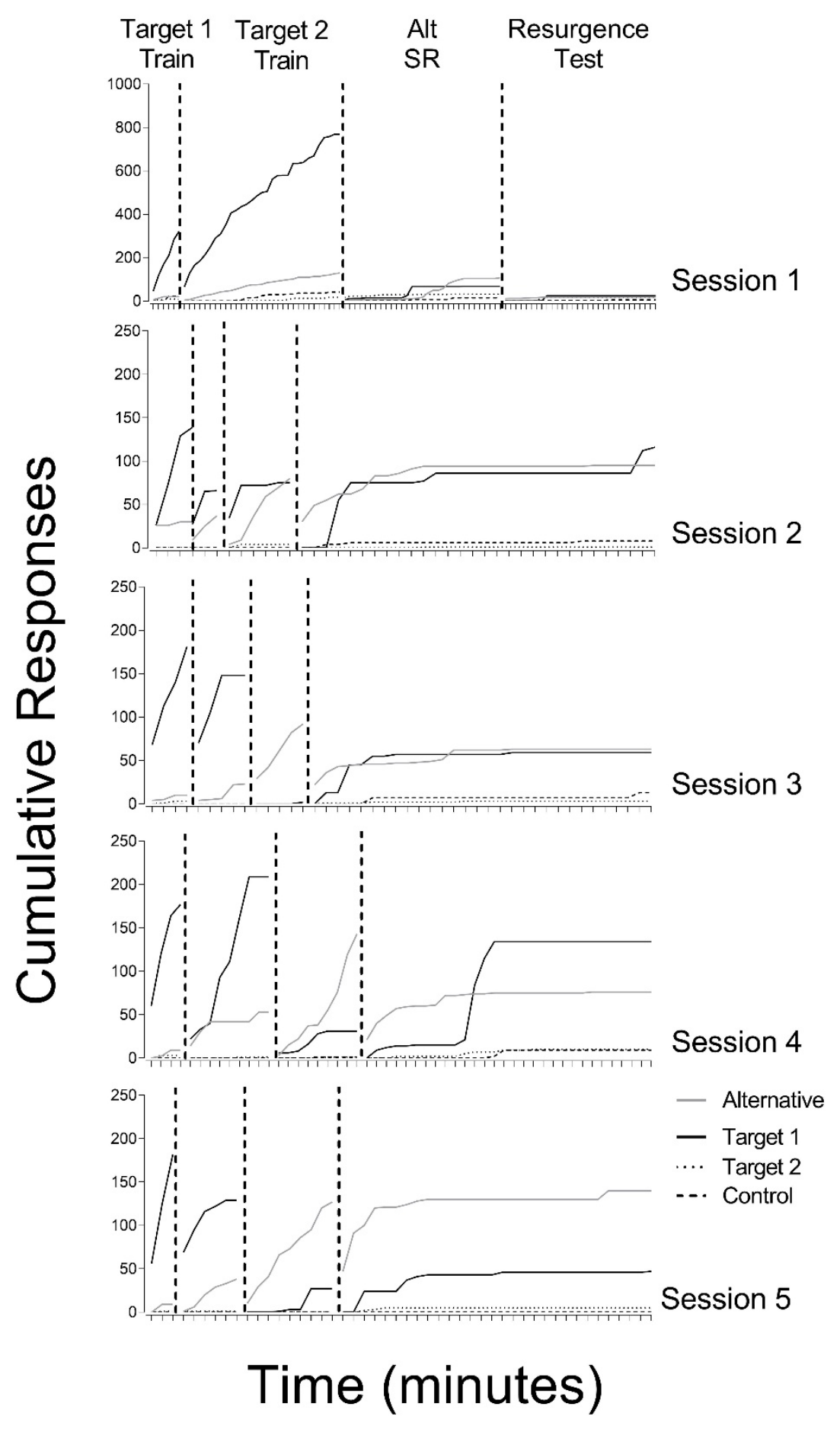

Figure B2. Cumulative record of Alternative, Target-1, Target-2, and Control responses during each session of Experiment $2 b$ for Rat 32. Note the different y-axis scale for Session 1. 

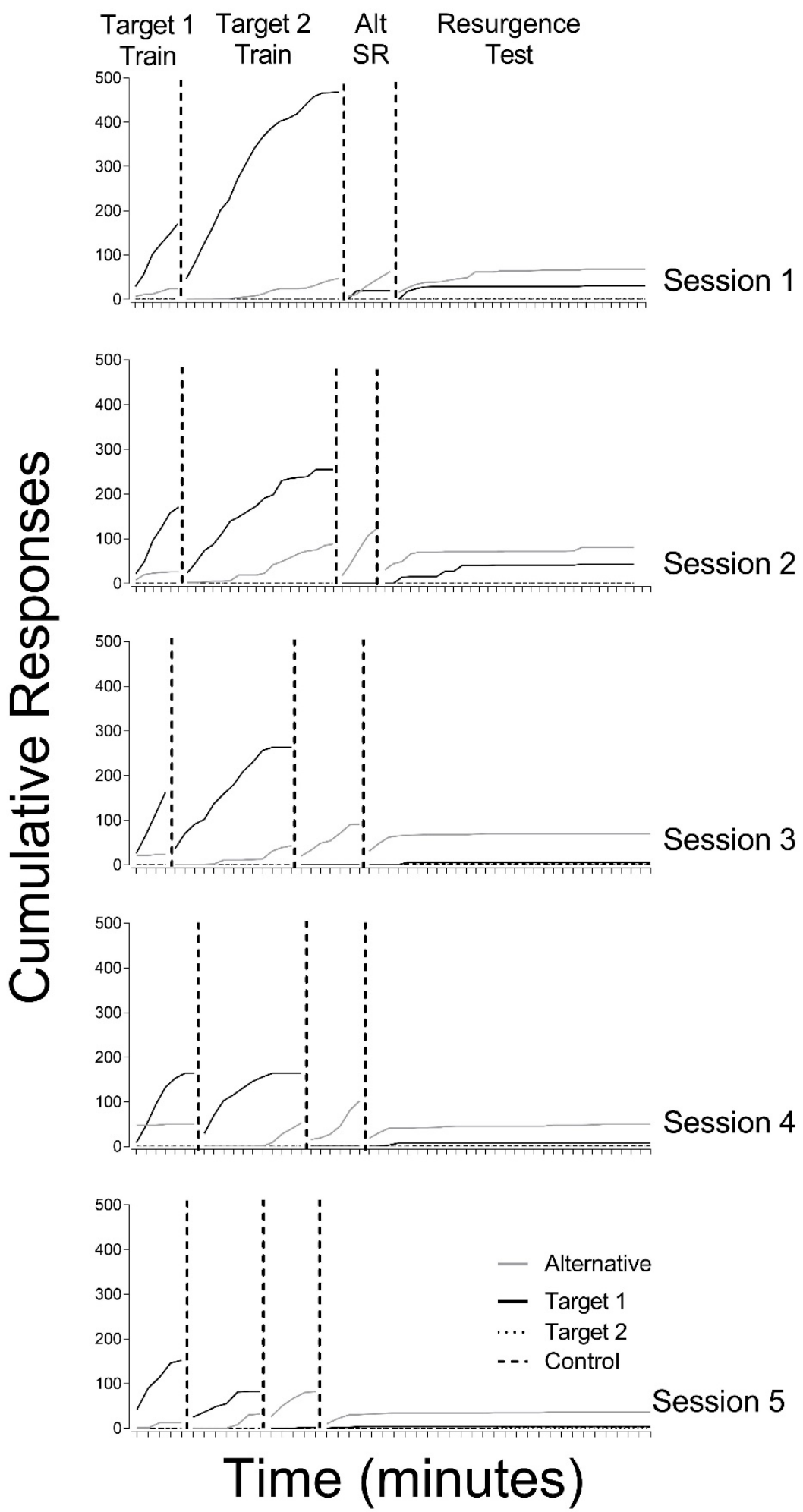

Figure B3. Cumulative record of Alternative, Target-1, Target-2, and Control responses during each session of Experiment $2 b$ for Rat 33. 


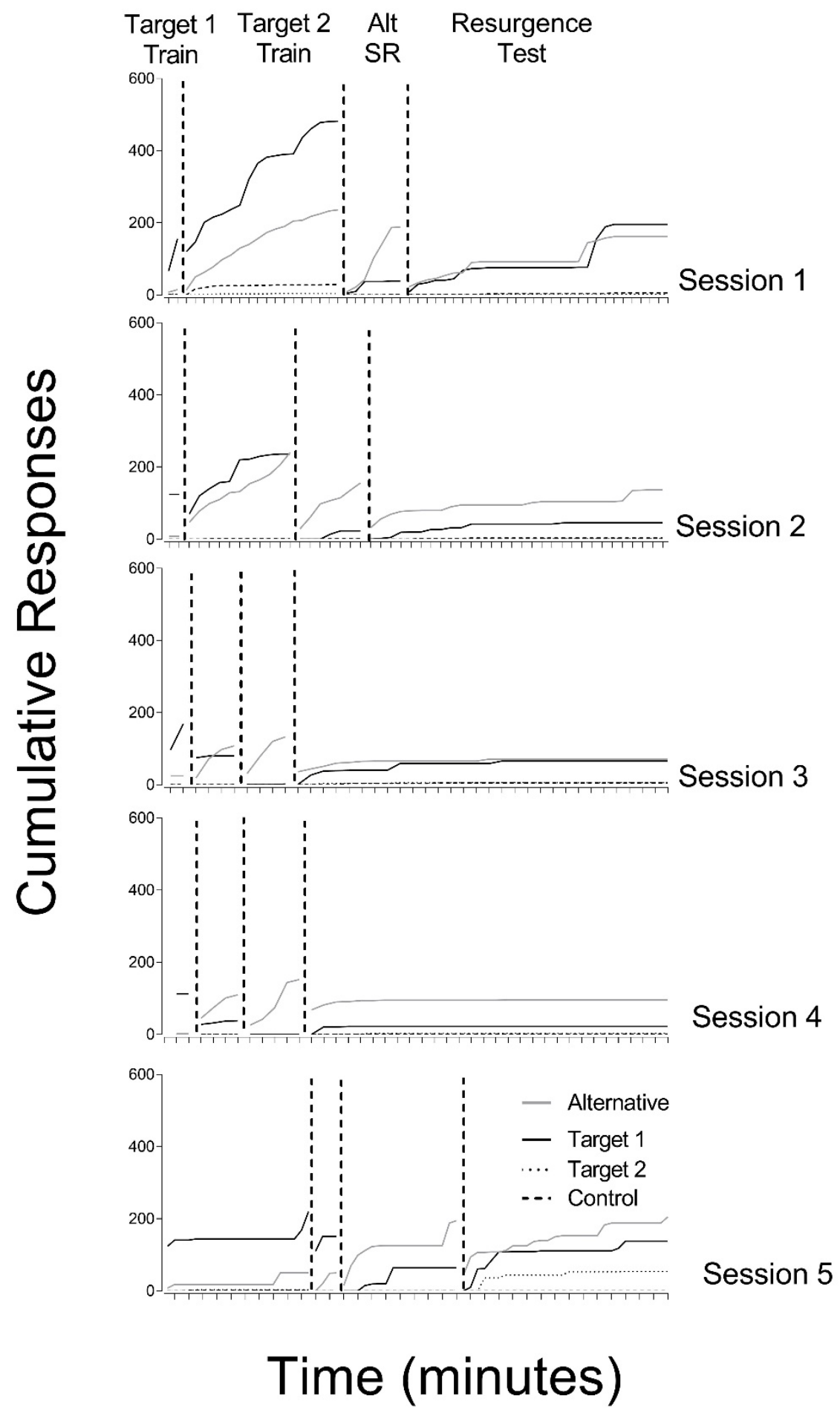

Figure B4. Cumulative record of Alternative, Target-1, Target-2, and Control responses during each session of Experiment $2 b$ for Rat 34. 